\title{
Strictness Types: An Inference Algorithm and an Application
}

\author{
Torben Amtoft \\ Computer Science Department \\ Aarhus University, Ny Munkegade, DK-8000 Arhus C, \\ Denmark \\ internet: tamtoft@daimi.aau.dk
}

August 10, 1993

\begin{abstract}
This report deals with strictness types, a way of recording whether a function needs its argument(s) or not. We shall present an inference system for assigning strictness types to expressions and subsequently we transform this system into an algorithm capable of annotating expressions with strictness types. We give an example of a transformation which can be optimized by means of these annotations, and finally we prove the correctness of the optimized transformation - at the same time proving the correctness of the annotation.

Everything has been implemented; documentation can be found in appendix.
\end{abstract}

\section{Introduction}

\section{$1.1 \quad$ Strictness Types}

Strictness analysis is the task of detecting whether a function needs its arguments. Recent years have seen many approaches to strictness analysis, 
most based on abstract interpretation - the starting point being the work of Mycroft [Myc80] which was extended to higher order functions by Burn, Hankin and Abramsky [BHA86]. These analyses have been proved correct in the following sense: if $f^{\#}$ is the abstract denotation of the function $f$, and if $f^{\#}(0)=0$ (0 denoting the bottom value in the abstract domain), then $f(\perp)=\perp$ (in the concrete domain) - that is, the call of $f$ will not terminate if its argument does not terminate ${ }^{1}$.

Kuo and Mishra [KM89] presented a type system where types $t$ are formed from 0 (denoting non-termination), 1 (denoting non-termination or termination, i.e. any term) and $t_{1} \rightarrow t_{2}$. Accordingly, if it is possible to assign a function the type $0 \rightarrow 0$ we know that the function is strict. This system, however, is strictly weaker than one based on abstract interpretation - on the other hand, Jensen [Jen91] proves that if conjunction types are added to the type system one gets a type system with the same power as abstract interpretation. To get an intuitive feeling of what's going on, consider a function $f$ with abstract denotation defined by $f(0)(1)=0, f(1)(0)=0, f(1)(1)=1$ (and by monotonicity hence also $f(0)(0)=0$ ). Such a function needs both of its arguments, and accordingly it has type $0 \rightarrow 1 \rightarrow 0$ as well as type $1 \rightarrow 0 \rightarrow 0$.

Wright has proposed alternative type systems [Wri91] and [Wri92]. The idea is to annotate the arrows: if a function can be assigned type Int $\rightarrow_{0}$ Int this means that the function is strict, whereas a type Int $\rightarrow_{1}$ Int doesn't tell anything about strictness properties. It should be clear that this is weaker than abstract interpretation, as Int $\rightarrow_{0}$ Int corresponds to two functions on the abstract domain: the identity function and the zero function. On the other hand, in some cases the method is more powerful than the one of [KM89]: a function which needs both of its arguments (cf. the above) can be assigned type Int $\rightarrow_{0}$ Int $\rightarrow_{0}$ Int.

In Sect. 3 we are going to present a type inference system based on Wright's idea, and by means of a few examples we shall illustrate the strengths and weaknesses of the system. A proof of the "semantic correctness" of the inference system will not appear before Sect. 6 where we shall prove the overall correctness of the system and a transformation exploiting the strictness information.

\footnotetext{
${ }^{1}$ This is not exactly equivalent to saying that " $f$ needs its argument", as $f$ may loop without ever looking at its argument.
} 
An ordering on strictness types, monotone in covariant position and antimonotone in contravariant position, will be imposed. Thus e.g. (Int $\left.\rightarrow_{1} \operatorname{lnt}\right)$ $\rightarrow_{0}$ Int $\leq\left(\operatorname{Int} \rightarrow_{0}\right.$ Int $) \rightarrow_{1}$ Int. So if $t_{1} \leq t_{2}$ then $t_{1}$ carries more information than $t_{2}$. The inference system will have a "subsumption rule", stating that if an expression has a type then it also has any greater type - thus allowing one to "forget information".

\subsection{An Inference Algorithm}

Section 4 is devoted to transforming the type inference system from Sect. 3 into an algorithm. As we want to concentrate upon strictness aspects and not upon type inference in general, we shall assume that the underlying type (such as e.g. Int $\rightarrow$ Int) has been given in advance. The first step is to "inline" the subsumption rule into the other rules, thus making the system "syntax-directed". Next we rewrite the system into one using constraints. As a result we get a system with the property that for any expression $e$ it is straight-forward to assign $e$ a typing where the arrows are annotated by strictness variables (variables which can assume the values 0 and 1), at the same time generating a set of constraints among these strictness variables. We are thus left with the problem of solving those constraints.

In type inference one is usually interested in finding a "principal type" such that all other valid typings can be found as substitution instances of this type. This is also the approach taken in [Wri91]. In our framework (which in this respect differs from Wright's), we would like to find a "least type" (wrt. the ordering $\leq$ ). However, in general no least typing exists as can be seen from the term twice $=\lambda f . \lambda x \cdot f(f(x))$ which has type $\left(\right.$ Int $\rightarrow_{0}$ Int $)$ $\rightarrow_{0}\left(\right.$ Int $\rightarrow_{0}$ Int $)$ and type $\left(\right.$ Int $\rightarrow_{1}$ Int $) \rightarrow_{0}\left(\right.$ Int $\rightarrow_{1}$ Int $)$ but not type (Int $\rightarrow_{1}$ Int $) \rightarrow_{0}\left(\operatorname{Int} \rightarrow_{0} \operatorname{lnt}\right)$. On the other hand, this example suggests that for each choice of assignments to the arrows occurring in contravariant positions there exists a least assignment to the arrows occurring in covariant position. This motivates the definition of a normalized set of constraints, which (loosely speaking) is a constraint set where each constraint is of form $\vec{b}^{+} \geq g\left(\vec{b}^{-}\right)$ where $\vec{b}^{+}\left(\vec{b}^{-}\right)$are strictness variables occurring in covariant (contravariant) position, and where $g$ is a monotone function.

We shall see that it is possible to normalize a constraint set "on the 
fly", i.e. during a traversal from leaves to root in the proof tree. Thus the conjecture above is true; once the annotations of arrows in contravariant position is fixed there exists a least annotation of the covariant arrows.

The usual approach to constraint solving is to collect them all and then solve them - which approach is the best one algorithmically is hard to say; we shall not address this issue.

The normalization algorithm employs some techniques which we think might be new - on the other hand, since constraint solving appears in numerous contexts it is quite possible that similar approaches exist in the literature.

\subsection{Tanslating from CBN to CBV}

Call-by-name (CBN) evaluation of the $\lambda$-calculus (and especially the variant known as "lazy evaluation") has been widely praised (e.g. in [Hug89]) because it makes programming a much more convenient task (another advantage is that referential transparency holds). On the other hand, as most implementations are call-by-value (CBV) one has to find means for translating from CBN to CBV. The naive approach (as presented e.g. in [DH92]) is to "thunkify" all arguments to applications, that is we have the following translation $T$ :

- An abstraction $\lambda x . e$ translates into $\lambda x . T(e)$;

- An application $e_{1} e_{2}$ translates into $T\left(e_{1}\right)\left(\lambda x \cdot T\left(e_{2}\right)\right.$ ) (where $x$ is a fresh variable) - that is, the evaluation of the argument is suspended ("thunkified");

- A variable $x$ translates into $(x d)$ (where $d$ is a "dummy" argument) since $x$ will become bound to a suspension $x$ must be "dethunkified".

This is clearly suboptimal since if $e_{1}$ is strict there is no need to thunkify its argument - this observation being the motivation for e.g. Mycroft's work on strictness analysis. Accordingly, in Sect. 5 we shall present a translation from CBN to CBV which exploits the information present in the strictness types. This translation is essentially similar to the one given by Danvy and Hatcliff [DH93]. 


\subsection{Proving the Tanslation Correct}

The optimized translation from CBN to CBV is folklore - but a correctness proof is certainly not. For instance, the translation presented in [DH93] is not proved correct, and even though the strictness analysis presented in [BHA86] is proved correct (in the sense that the abstract semantics actually abstracts the concrete semantics) the correctness of an optimization based on this analysis has not been proved. The same remarks apply to e.g. [Wri91] and reflect a quite general phenomenon, cf. the claims made in [Wan93, p. 137]:

The goal of flow analysis is to annotate a program with certain propositions about the behavior of that program. One can then apply optimizations to the program that are justified by those propositions. However, it has proven remarkably difficult to specify the semantics of those propositions in a way that justifies the resulting optimizations.

In [Wan93], Wand proved the correctness of a partial evaluator which exploits binding time information. In Sect. 6 we follow this trend, within the context defined in Sect. 5 (i.e. a CBN-to-CBV translator exploiting strictness information). Also something similar can be found in [Lan92] where the correctness of a code generation exploiting strictness information is proved.

The basic idea in expressing the correctness of the translation from Sect. 5 is to use logical relations (on closed terms): $q \sim_{t} q^{\prime}$ should be interpreted as stating that $q^{\prime}$ is a correct translation of $q$. If $t$ is a base type, $q \sim_{t} q^{\prime}$ holds iff $q$ when evaluated by CBN yields the same result as $q^{\prime}$ when evaluated by CBV (in particular $q$ loops by CBN iff $q^{\prime}$ loops by CBV). For a strict function type $t=t_{1} \rightarrow_{0} t_{2}, q \sim_{t} q^{\prime}$ means that whenever $q_{1} \sim_{t_{1}} q_{1}^{\prime}$; then $q q_{1} \sim_{t_{2}} q^{\prime} q_{1}^{\prime}$. For a non-strict function type $t=t_{1} \rightarrow t_{2}, q \sim_{t_{1}} q^{\prime}$ means that whenever $q_{1} \sim_{t_{1}} q_{1}^{\prime}$ then $q q_{1} \sim_{t_{2}} q^{\prime}\left(\lambda x \cdot q_{1}^{\prime}\right)(x$ a fresh variable $)$.

The noteworthy point is that the fact that a function actually is strict is not expressed using some relationship between concrete/abstract domains, but simply by stating that it is correct not to thunkify its argument! This corresponds to the claim in [Wan93, p. 137]:

This work suggests that the proposition associated with a flow 
analysis can simply be that "the optimization works".

The extension of the correctness predicate to open terms is rather straightforward - and so is the correctness proof, the only tricky point being how to cope with recursion.

\subsection{An Implementation}

The type inference algorithm from Sect. 4 and the translation algorithm from Sect. 5 has been implemented in Miranda ${ }^{2}$. The user interface is as follows:

- the user writes a $\lambda$-expression, and provides the underlying type of the bound variables;

- the user provides the annotation of the contravariant arrows in the overall type;

- then the system produces the least valid annotation of the remaining arrows, and translates the original expression into a CBV-equivalent expression.

The system is documented in Appendix A.

\subsection{Acknowledgements}

The author is supported by the DART-project funded by the Danish Research Council.

The work reported here evolved from numerous discussions with Hanne Riis Nielson and Flemming Nielson. Also thanks to Jens Palsberg for useful feedback.

\footnotetext{
${ }^{2}$ Miranda is a trademark of Research Software Limited.
} 


\section{Preliminaries}

\section{Expressions}

An expression is either a constant $c$; a variable $x$; an abstraction $\lambda x . e$; an application $e_{1} e_{2}$; a conditional if $e_{1} e_{2} e_{3}$ or a recursive definition rec $f e$.

The reason for not making if a constant (thereby making it possible to dispense with the conditional) is that if is a non-strict constant and hence requires special treatment.

\section{Types}

The set of (underlying) types will be denoted $\mathcal{T}$; such a type is either a base type (Int, Bool, Unit etc.) or a function type $t_{1} \rightarrow t_{2}$. Base will denote some base type.

An iterated base type is either Base or of form Base $\rightarrow t$ where $t$ is an iterated base type. We shall assume that there exists a function $C t$ which assigns iterated base types to all constants (we will expect to have $C t(7)=$ Int, $C t(+)=$ Int $\rightarrow$ int $\rightarrow$ Int etc.).

$$
\begin{array}{ll}
\Gamma \vdash c: C t(c) & \left(\Gamma_{1},(x: t), \Gamma_{2}\right) \vdash x: t \\
\frac{\left(\left(x: t_{1}\right), \Gamma\right) \vdash e: t}{\Gamma \vdash \lambda x \cdot e: t_{1} \rightarrow t} & \frac{\Gamma \vdash e_{1}: t_{2} \rightarrow t_{1}, \Gamma \vdash e_{2}: t_{2}}{\Gamma \vdash e_{1} e_{2}: t_{1}} \\
\frac{\Gamma \vdash e_{1}: \text { Bool, } \Gamma \vdash e_{2}: t, \Gamma \vdash e_{3}: t}{\Gamma \vdash\left(\text { if } e_{1} e_{2} e_{3}\right): t} & \frac{((f: t), \Gamma) \vdash e: t}{\Gamma \vdash(\operatorname{rec} f e): t}
\end{array}
$$

Figure 1: An inference system for (underlying) types. 
In Fig. 1 we present a type inference system, where inferences are of form $\Gamma \vdash e: t$. Here $\Gamma$ is an environment assigning types to (a superset of) the free variables of $e ; \Gamma$ will be represented as a list of pairs of form $(x: t)^{34}$. For closed expressions $q$ it makes sense to say that $q$ is of type $t$, since if $\Gamma \vdash q: t$ then also $\Gamma^{\prime} \vdash q: t$ for any $\Gamma^{\prime}$.

\section{Semantics}

We say that an expression is in weak head normal form (WHNF) if it is either a constant $c$ or of form $\lambda x$.e. As no constructors are present in the language, this choice of normal form will be suitable for CBV as well as for CBN.

We define a SOS for call-by-name (Fig. 2) and a SOS for call-by-value (Fig. 3), with inferences of form $q \Rightarrow_{N} q^{\prime}$ resp. $q \Rightarrow_{V} q^{\prime}$. Here $q$ and $q^{\prime}$ are closed expressions. We assume the existence of a function Applycon such that for two constants $c_{1}$ and $c_{2}$, Applycon $\left(c_{1}, c_{2}\right)$ either yields another constant $c$ such that if $C t\left(c_{1}\right)=$ Base $\rightarrow t, C t\left(c_{2}\right)=$ Base then $C t(c)=t$ or the expression $c_{1} c_{2}$ itself (to model errors). For instance, Applycon $(+, 4)$ could be the constant $+_{4}$, where Applycon $\left(+_{4}, 3\right)$ is the constant 7 . To model that division by zero is illegal we let e.g. $\operatorname{Applycon}\left(/{ }_{7}, 0\right)=\left(/{ }_{7}, 0\right)$.

We have the following (standard) result (which exploits that all constants are of iterated base type, as otherwise $c(\lambda x . e)$ might be well-typed but stuck). We need the extra assumption that if $C t(c)=$ Bool then $c=$ True or $c=$ False.

Fact 2.1 Suppose (with $q$ closed) $\Gamma \vdash q: t$. Then either $q$ is in WHNF, or there exists unique $q^{\prime}$ such that $q \Rightarrow_{N} q^{\prime}$ and such that $\Gamma \vdash q^{\prime}: t$.

Similarly for $\Rightarrow_{V}$.

We will introduce a "canonical" looping term $\Omega$, defined by $\Omega=$ ret $f f$. There exists no $q$ in WHNF such that $\Omega \Rightarrow_{N}^{*} q\left(\right.$ or $\left.\Omega \Rightarrow_{V}^{*} q\right)$, but for all types $t$ (and all $\Gamma$ ) we have $\Gamma \vdash \Omega: t$.

\footnotetext{
${ }^{3}$ To concatenate e.g. the list $l_{1}$ with the list $l_{2}$ we shall write $\left(l_{1}, l_{2}\right)$.

${ }^{4}$ In case of multiple occurrences of some variable $x$, it is the leftmost occurrence which "counts" - we are not going to bother further about that.
} 


$$
\begin{aligned}
& (\lambda x . e) q \Rightarrow_{N} e[q / x] \quad \frac{q_{1} \Rightarrow_{N} q_{1}^{\prime}}{q_{1} q_{2} \Rightarrow_{N} q_{1}^{\prime} q_{2}} \\
& c_{1} c_{2} \Rightarrow_{N} \text { Applycon }\left(c_{1}, c_{2}\right) \frac{q_{2} \Rightarrow_{N} q_{2}^{\prime}}{c q_{2} \Rightarrow_{N} c q_{2}^{\prime}} \\
& \text { if True } q_{2} q_{3} \Rightarrow_{N} q_{2} \quad \text { if False } q_{2} q_{3} \Rightarrow_{N} q_{3} \\
& \frac{q_{1} \Rightarrow_{N} q_{1}^{\prime}}{\text { if } q_{1} q_{2} q_{3} \Rightarrow_{N} \text { if } q_{1}^{\prime} q_{2} q_{3}} \quad \text { rec } f e \Rightarrow_{N} e[(\operatorname{rec} f e) / f]
\end{aligned}
$$

Figure 2: A SOS for CBN.

$$
\begin{aligned}
& (\lambda x . e) q \Rightarrow_{V} e[q / x], \text { if } q \text { in WHNF } \frac{q_{1} \Rightarrow_{V} q_{1}^{\prime}}{q_{1} q_{2} \Rightarrow_{V} q_{1}^{\prime} q_{2}} \\
& c_{1} c_{2} \Rightarrow_{V} \operatorname{Applycon}\left(c_{1}, c_{2}\right) \quad \frac{q_{2} \Rightarrow_{V} q_{2}^{\prime}}{q_{1} q_{2} \Rightarrow_{V} q_{1} q_{2}^{\prime}}, \text { if } q_{1} \text { in WHNF } \\
& \text { if True } q_{2} q_{3} \Rightarrow_{V} q_{2} \quad \text { if False } q_{2} q_{3} \Rightarrow_{V} q_{3} \\
& \frac{q_{1} \Rightarrow_{V} q_{1}^{\prime}}{\text { if } q_{1} q_{2} q_{3} \Rightarrow_{V} \text { if } q_{1}^{\prime} q_{2} q_{3}} \quad \quad \text { rec } f e \Rightarrow_{V} e[(\operatorname{rec} f e) / f]
\end{aligned}
$$

Figure 3: A SOS for CBV.

\section{Thunkification and Dethunkification}

We shall use the following notation: if $t$ is a type in $\mathcal{T},[t]$ is a shorthand for Unit $\rightarrow t$.

If $e$ is an expression, let $\underline{e}$ be a shorthand for $\lambda x . e$, where $x$ is a fresh variable.

If $e$ is an expression, let $\mathcal{D}(e)$ be a shorthand for $e d$ where $d$ is a dummy constant of type Unit.

Fact 2.2 If $\Gamma \vdash e: t$, then $\Gamma \vdash c:[t]$. If $\Gamma \vdash \underline{e}:[t]$, then $\Gamma \vdash \mathcal{D}(e): t$. 
For all $e, \mathcal{D}(\underline{e}) \Rightarrow_{N} e$ and $\mathcal{D}(\underline{e}) \Rightarrow_{V} e$.

\section{Strictness Types}

In this section we shall augment types with strictness information, that is annotate the arrows. The set of strictness types, $\mathcal{T}_{s a}$, is defined as follows: a strictness type $t$ is either a base type Base or a strict function type $t_{1} \rightarrow_{0} t_{2}$ (denoting that we know that the function is strict) or a general function type $t_{1} \rightarrow_{1} t_{2}$ (denoting that we do not know whether the function is strict).

It will be convenient to introduce some notation: if $t$ is a (standard) type in $\mathcal{T}$, and if $\vec{b}^{+}$and $\vec{b}^{-}$are vectors of 0 or 1's, then $t\left[\vec{b}^{+}, \vec{b}^{-}\right]$denotes $t$ where all covariant arrows are marked (from left to right) as indicated by $\vec{b}^{+}$and where all contravariant arrows are marked (from left to right) as indicated by $\vec{b}^{-}$. More formally, we have

- Base $[(),()]=$ Base;

- $\left(t_{1} \rightarrow t_{2}\right)\left[\left(\vec{b}_{1}^{+}, b^{+}, \vec{b}_{2}^{+}\right),\left(\vec{b}_{1}^{-}, \vec{b}_{2}^{-}\right)\right]=t_{1}\left[\vec{b}_{1}^{-}, \vec{b}_{1}^{+}\right] \rightarrow_{b^{+} t_{2}\left[\vec{b}_{2}^{+}, \vec{b}_{2}^{-}\right]}$

Example 3.1 With $t=((\ln t \rightarrow \ln t) \rightarrow \ln \mathrm{t}) \rightarrow((\ln t \rightarrow \ln \mathrm{t}) \rightarrow \ln \mathrm{t})$, we have

$$
t\left[\left(b_{1}, b_{2}, b_{3}\right),\left(b_{4}, b_{5}\right)\right]=\left(\left(\operatorname{lnt} \rightarrow_{b_{1}} \operatorname{lnt}\right) \rightarrow_{b_{4}} \operatorname{lnt}\right) \rightarrow_{b_{2}}\left(\left(\operatorname{lnt} \rightarrow_{b_{5}} \operatorname{lnt}\right) \rightarrow_{b_{3}} \operatorname{lnt}\right)
$$

If $\Gamma=\left(\left(x_{1}: t_{1}\right) \ldots\left(x_{n}: t_{n}\right)\right)$ then

$$
\Gamma\left[\left(\vec{b}_{1}^{-}, \ldots, \vec{b}_{n}^{-}\right),\left(\vec{b}_{1}^{+}, \ldots, \vec{b}_{n}^{+}\right)\right]=\left(\left(x_{1}: t_{1}\left[\vec{b}_{1}^{-}, \vec{b}_{1}^{+}\right]\right), \ldots,\left(x_{n}: t_{n}\left[\vec{b}_{n}^{-}, \vec{b}_{n}^{+}\right]\right)\right)
$$

\section{An Ordering Relation}

We shall impose an ordering $\leq$ on strictness types, defined by stipulating that $t_{1} \rightarrow_{b} t_{2} \leq t_{1}^{\prime} \rightarrow_{b^{\prime}} t_{2}^{\prime}$; iff $t_{1}^{\prime} \leq t_{1}, b \leq b^{\prime}$ and $t_{2} \leq t_{2}^{\prime}$, and by stipulating that Int $\leq$ Int etc. $t \leq t^{\prime}$ means that $t$ is more informative than $t^{\prime}$; for 
instance it is more informative to know that a function is of type Int $\rightarrow_{0} \operatorname{lnt}$ than to know that it is of type Int $\rightarrow_{1}$ Int. Similarly, it is more informative to know that a function is of type (Int $\rightarrow_{1}$ Int) $\rightarrow_{0}$ (Int $\rightarrow_{0}$ Int) (it maps arbitrary functions into strict functions) than to know that is is of type (Int $\rightarrow_{0}$ Int $\rightarrow_{0}$ (Int $\rightarrow_{0}$ Int) (it maps strict functions into strict functions).

Fact $3.2 t\left[\vec{b}_{1}^{+}, \vec{b}_{1}^{-}\right] \leq t\left[\vec{b}_{2}^{+}, \vec{b}_{2}^{-}\right]$iff $\vec{b}_{1}^{+} \leq \vec{b}_{2}^{+}$and $\vec{b}_{2}^{-} \leq \vec{b}_{1}^{-}$(pointwise).

\section{The Relation Between $\mathcal{T}$ and $\mathcal{T}_{s a}$}

We define a mapping $\mathrm{E}$ from $\mathcal{T}_{\text {sa }}$ into $\mathcal{T}$, which just removes annotations from arrows - that is, we have $\mathrm{E}($ Base $)=$ Base, $\mathrm{E}\left(t_{1} \rightarrow_{0} t_{2}\right)=\mathrm{E}\left(t_{1}\right) \rightarrow \mathrm{E}\left(t_{2}\right)$, $\mathrm{E}\left(t_{1} \rightarrow{ }_{1} t_{2}\right)=\mathrm{E}\left(t_{1}\right) \rightarrow \mathrm{E}\left(t_{2}\right)$. Clearly, $\mathrm{E}\left(t\left[\vec{b}^{+}, \vec{b}^{-}\right]\right)=t$. And if $t_{1} \leq t_{2}$, then $\mathrm{E}\left(t_{1}\right)=\mathrm{E}\left(t_{2}\right)$.

We have to extend $C t$ into $C T_{s a}$ a mapping from constants into strictness types, and doing so we shall exploit that all constants are strict (recall that the non-strict constant if has been given a special status). Accordingly, we define $C T_{s a}(c)=C t(c)[\overrightarrow{0},()]$.

\section{The Inference System}

In Fig. 4 we present an inference system for strictness types. A judgement is now of the form $\Gamma \vdash_{s a} e: t, W$. Here

- $\Gamma$ is an environment (represented as a list) assigning strictness types to variables;

- $e$ is an expression such that if $x$ is a free variable of $e(x \in \mathrm{FV}(e))$ then $\Gamma(x)$ is defined;

- $t$ is a strictness type;

- $W$ maps the domain of $\Gamma$ into $\{0,1\}$. It may be helpful to think of $W$ as follows: if $W(x)=0$ then $x$ is needed in order to evaluate $e$ to "head normal form". 
Some notation: if $\Gamma=\left(\left(x_{1}: t_{1}\right) \ldots\left(x_{n}: t_{n}\right)\right)$ and if $W\left(x_{i}\right)=b_{i}$ we shall often write $W=\left(b_{1} \ldots b_{n}\right)$. Also, we shall sometimes write $W=\left\{x_{i} \mid b_{i}=0\right\}$ (i.e. identify $W$ with the set of variables on which $W$ assumes the value 0 ). In the natural way, $\mathrm{E}$ is extended to work on environments.

Now a brief explanation of the inference system: the first inference rule (the "subsumption rule") is non-structural and expresses the ability to forget information: if an expression has type $t$ and needs the variables in $W$, it also has a more imprecise type and will also need a subset of $W$. The application of this rule might for instance be needed in order to assign the same type to the two branches in a conditional. The rule for variables expresses (among other things) that in order to evaluate $x$ it is necessary to evaluate $x(!)$ but no other variables are needed. The rule for abstractions (among other things) says that if $x$ is among the variables needed by $e$ then $\lambda x$.e can be assigned a strict type $\left(\rightarrow_{0}\right)$, otherwise not. The rule for applications (among other things) says that the variables needed to evaluate $e_{1}$ are also needed to evaluate $e_{1} e_{2}$; and if $e_{1}$ is strict then the variables needed to evaluate $e_{2}$ will also be needed to evaluate $e_{1} e_{2}$. The rule for conditionals (among other things) says that if a variable is needed to evaluate the test then it is also needed to evaluate the whole expression; and also if a variable is needed in order to evaluate both branches it will be needed to evaluate the whole expression.

Notice that ()$\vdash_{s a} \Omega: t,()$ for all strictness types $t$.

An expression which can be assigned a strictness type can also be assigned an underlying type:

Fact 3.3 Suppose $\Gamma \vdash_{s a} e: t, W$. Then $\mathrm{E}(\Gamma) \vdash e: \mathrm{E}(t)$.

Conversely, an expression which can be assigned an underlying type can also be assigned at least one strictness type:

Fact 3.4 Suppose $\Gamma \vdash e: t$. Then $\Gamma[\overrightarrow{1}, \overrightarrow{1}] \vdash_{s a} e: t[\overrightarrow{1}, \overrightarrow{1}], \overrightarrow{1}$.

Proof: An easy induction in the proof tree. In the case of a constant $c$, we have to use the subsumption rule and exploit that $C T_{s a}(c)=$ $C t(c)[\overrightarrow{0},()] \leq C t(c)[\overrightarrow{1},()]$. 


$$
\begin{aligned}
& \frac{\Gamma \vdash_{s a} e: t, W}{\Gamma \vdash_{s a} e: t^{\prime}, W^{\prime}} \text { if } t^{\prime} \geq t, W^{\prime} \geq W \\
& \Gamma \vdash_{s a} c: C T_{s a}(c), \overrightarrow{1} \\
& \left(\Gamma_{1},(x: t), \Gamma_{2}\right) \vdash_{s a} x: t,(\overrightarrow{1}, 0, \overrightarrow{1}) \\
& \frac{\left(\left(x: t_{1}\right), \Gamma\right) \vdash_{s a} e: t,(b, W)}{\Gamma \vdash_{s a} \lambda x \cdot e: t_{1} \rightarrow{ }_{b} t, W} \\
& \frac{\Gamma \vdash_{s a} e_{1}: t_{2} \rightarrow_{b} t_{1}, W_{1} \Gamma \vdash_{s a} e_{2}: t_{2}, W_{2}}{\Gamma \vdash_{s a} e_{1} e_{2}: t_{1}, W} \\
& \text { if } W(x)=W_{1}(x) \Pi\left(b \sqcup W_{2}(x)\right) \text { for all } x \\
& \frac{\Gamma \vdash_{s a} e_{1}: \text { Bool, } W_{1} \Gamma \vdash_{s a} e_{2}: t, W_{2} \Gamma \vdash_{s a} e_{3}: t, W_{3}}{\Gamma \vdash-\vdash_{s a}\left(\text { if } e_{1} e_{2} e_{3}\right): t, W} \\
& \text { if } W(x)=W_{1}(x) \sqcap\left(W_{2}(x) \sqcup W_{3}(x)\right) \text { for all } x \\
& \frac{((f: t), \Gamma) \vdash_{s a} e: t,(b, W)}{\Gamma \vdash_{s a}(\text { rec } f e): t, W}
\end{aligned}
$$

Figure 4: An inference system for strictness types. 
Example 3.5 Consider the function $f$ defined by rec $f \lambda x . \lambda y . \lambda z$.e where $e=$ if $(z=0)(x+y)(f y x(z-1)) . f$ is strict in all its arguments, but this cannot be inferred by the type system from [KM89] due to the lack of conjunction types. In our system, however, we have

$$
() \vdash_{s a} \text { rec } f \lambda x . \lambda y . \lambda z . e: \operatorname{lnt} \rightarrow{ }_{0} \operatorname{Int} \rightarrow_{0} \operatorname{lnt} \rightarrow_{0} \operatorname{lnt}, \emptyset
$$

This is because we - with $\Gamma_{1}=\left(\left(f: \operatorname{lnt} \rightarrow_{0} \operatorname{Int} \rightarrow_{0} \operatorname{Int} \rightarrow_{0} \operatorname{Int}\right)\right)-$ have

$$
\Gamma_{1} \vdash_{s a} \lambda x . \lambda y \cdot \lambda z . e: \operatorname{lnt} \rightarrow_{0} \operatorname{lnt} \rightarrow_{0} \ln t \rightarrow_{0} \operatorname{lnt}, \emptyset
$$

which again is because we - with $\Gamma_{2}=\left((z: \operatorname{lnt}),(y: \operatorname{lnt}),(x: \operatorname{lnt}), \Gamma_{1}\right)-$ have

$$
\Gamma_{2} \vdash_{s a} \text { if }(z=0)(x+y)(f y x(z-1)): \operatorname{Int}\{x, y, z\}
$$

This follows from the fact that $\Gamma_{2} \vdash_{s a}(z=0)$ : Bool $\{z\}$ and $\Gamma_{2} \vdash_{s a}(x+y)$ : Int $\{x, y\}$ and

$$
\Gamma_{2} \vdash_{s a}(f y x(z-1)): \operatorname{Int}\{x, y, z\}
$$

The latter follows since e.g. $\Gamma_{2} \vdash_{s a}(f y): \operatorname{lnt} \rightarrow_{0} \operatorname{lnt} \rightarrow_{0} \operatorname{lnt},\{y\}$.

Example 3.6 Our analysis is not very good at handling recursive definitions with free variables. To see this, consider the function $g$ given

$$
\lambda y . \text { rec } f \lambda x \text {.if }(x=0) y(f(x-1))
$$

Clearly $g e_{1} e_{2}$ will loop if $e_{1}$ loops, so the analysis ought to conclude that $g$ has strictness type Int $\rightarrow_{0} \operatorname{Int} \rightarrow_{0}$ Int. However, we can do no better than inferring that $g$ has strictness type Int $\rightarrow_{1} \ln t \rightarrow_{0} \operatorname{lnt}-$ this is because it is impossible to deduce $\ldots \vdash_{s a}(\operatorname{rec} f \ldots): \ldots,\{y\}$ which in turn is because it is impossible to deduce $\ldots \vdash_{s a}$ (if $\left.(x=0) y(f(x-1))\right): \ldots,\{x, y\}$. The reason for this is that we cannot record in $\Gamma(f)$ that $f$ needs $y$.

In order to repair on that, function arrows should be annotated not only with $0 / 1$ but also with which free variables are needed - at the cost of complicating the theory significantly. 


\section{An Inference Algorithm}

In this section we shall work on the inference system from Fig. 4 and transform it into an algorithm. This will be a two-stage process: first the inference system is transformed into an equivalent one using constraints; then an algorithm is given for solving those constraints. We shall assume that all subexpressions are (implicitly) annotated with an "underlying type" (belonging to $\mathcal{T}$ ) such that the expression is well-typed according to the rules in Fig. 1.

$$
\begin{aligned}
& \Gamma \vdash_{s a} c: t, \overrightarrow{1} \text { if } t \geq C T_{s a}(c) \\
& \left(\Gamma_{1},(x: t), \Gamma_{2}\right) \vdash_{s a} x: t^{\prime},(\overrightarrow{1}, b, \overrightarrow{1}) \text { if } t^{\prime} \geq t, b \geq 0 \\
& \frac{\left(\left(x: t_{1}\right), \Gamma\right) \vdash_{s a} e: t,(b, W)}{\Gamma \vdash_{s a} \lambda x \cdot e: t_{1} \rightarrow{ }_{b} t, W} \\
& \frac{\Gamma \vdash_{s a} e_{1}: t_{2} \rightarrow{ }_{b} t_{1}, W_{1} \Gamma \vdash_{s a} e_{2}: t_{2}, W_{2}}{\Gamma \vdash_{s a} e_{1} e_{2}: t_{1}, W} \\
& \text { if } W(x) \geq W_{1}(x) \sqcap\left(b \sqcup W_{2}(x)\right) \text { for all } x \\
& \frac{\Gamma \vdash_{s a} e_{1}: \text { Bool, } W_{1} \Gamma \vdash_{s a} e_{2}: t_{2}, W_{2} \Gamma \vdash_{s a} e_{3}: t_{3}, W_{3}}{\left.\Gamma \vdash_{s a} \text { (if } e_{1} e_{2} e_{3}\right): t, W} \\
& \text { if } t \geq t_{2}, t \geq t_{3}, W(x) \geq W_{1}(x) \sqcap\left(W_{2}(x) \sqcup W_{3}(x)\right) \text { for all } x \\
& \frac{((f: t), \Gamma) \vdash_{s a} e: t,(b, W)}{\Gamma \vdash_{s a}(\text { rec } f e): t^{\prime}, W} \text { if } t^{\prime} \geq t
\end{aligned}
$$

Figure 5: The result of inlining the subsumption rule.

\subsection{Getting a Constraint-Based Inference System}

The first step will be to "inline" the subsumption rule into (some of) the other rules. The result is depicted in Fig. 5.

Fact 4.1 A judgement is derivable in the system in Fig. 4 iff it is derivable in the system in Fig. 5 . 
ProOF: The "if"-part is an easy induction in the proof tree. For the "only if" -part, we need that if $\Gamma \vdash_{s a} e: t, W$ using Fig. 5 and $t \leq t^{\prime}, W \leq W^{\prime}$ then also $\Gamma \vdash_{s a} e: t^{\prime}, W^{\prime}$ using Fig. 5. This follows from a more general fact, which easily can be proved by induction in the proof tree: if $\Gamma_{s a} e: t, W$ using Fig. 5 and $\Gamma^{\prime} \leq \Gamma$ (pointwise), $t \leq t^{\prime}$ and $W \leq W^{\prime}$ then $\Gamma^{\prime} \vdash_{s a} e: t^{\prime}, W^{\prime}$ using Fig. 5.

It will be convenient to reformulate the system in Fig. 5, using the $t\left[\vec{b}^{+}, \vec{b}^{-}\right]$notation thus making annotations on arrows more explicit. This is done ${ }^{5}$ in Fig. 6; it is immediate to verify that this system is equivalent to the one in Fig. 5. A remark about covariant/contravariant position: the turnstile $\vdash$ acts like an $\rightarrow$, so if $t=\Gamma(x)$ then covariant positions in $t$ will be considered as appearing contravariantly in the judgement, and vice versa. On the other hand, something appearing in the range of $W$ is considered as being in covariant position in the judgement. We shall consistently use the convention that a superscript + (e.g. as in $\left.\vec{b}_{1}^{+}\right)$indicates something which appears on an arrow in covariant position in the judgement; whereas a superscript - (e.g. as in $\vec{b}_{2}^{-}$) indicates something which appears on an arrow in contravariant position in the judgement. It is important to notice that this can be done consistently, i.e. that polarity is always the same in the premise as in the conclusion.

We shall now introduce open strictness types: this is similar to strictness types except that the arrows are annotated not by 0's and 1's, but by a certain kind of variables to be called strictness variables. An open strictness type $t$, together with a mapping from the strictness variables in $t$ into $\{0,1\}$, in the natural way determines a strictness type.

Notice that in Fig. 6 the $\vec{b}^{+}$'s and the $\vec{b}^{-}$'s really are meta variables, ranging over 0 and 1 . By making them range over strictness variables we shall obtain a type inference system, depicted in Fig. 7, with judgements of form $\Gamma \vdash_{s a} e: t, W, C$. Here $\Gamma$ is an environment (represented as a list) mapping (program) variables into open strictness types; $t$ is an open strictness type; $W$ is a mapping (represented as a list) from program variables into strictness variables; and $C$ is a set of constraints among strictness variables.

\footnotetext{
${ }^{5}$ For space reasons we shall employ the convention that e.g. " $\Gamma \vdash_{s a} e_{1}: t_{1}, W_{1}$ and $e_{2}: t_{2}, W_{2}$ " means " $\Gamma \vdash_{s a} e_{1}: t_{1}, W_{1}$ and $\Gamma \vdash_{s a} e_{2}: t_{2}, W_{2}$ ".
} 


\subsection{Solving the Constraints}

The inference system in Fig. 7 does in the obvious way give rise to a deterministic algorithm, provided

1. we are able to find the underlying types (i.e. without strictness annotations) of all expressions;

2. we are able to solve the constraints generated.

As already mentioned we shall assume that 1 has been done in advance; so our aim will be to give an algorithm for solving the constraints generated by the system. Our approach will be to show how to normalize this system, thus showing that the solutions have a particular form. First some preliminaries:

\section{Strictness Expressions}

Strictness expressions will be built from strictness variables, $0,1, \sqcap$ and $\sqcup$. Thus a strictness expression $s$ in the natural way gives rise to a monotone function $g$ with domain the free variables of $s$ (and all monotone functions on finite domains can be represented by strictness expressions).

\section{Extended Constraints}

The constraints met in Fig. 7 are of form $\vec{b}_{1} \geq g\left(\vec{b}_{2}\right)$ or of form $\vec{b}_{1}=g\left(\vec{b}_{2}\right)$, with $g$ being a monotone function. We introduce a new kind of constraints, which besides using $\geq$ and $=$ also use a special symbol $\gg$. Intuitively, $b_{1} \gg g\left(b_{2}\right)$ "lies between" $b_{1}=g\left(b_{2}\right)$ and $b_{1} \geq g\left(b_{2}\right)$. More precisely, we have:

Definition 4.2 Let $N$ be an extended constraint system (i.e. possibly containing $\gg)$. Let $\phi$ be a mapping from the strictness variables of $N$ into $\{0,1\}$. We say that $\phi$ is a strong solution to $N$ iff $\phi$ is a solution to the system resulting from replacing all $\gg$ 's in $N$ by $=$; and we say that $\phi$ is a weak sohtion to $N$ iff $\phi$ is a solution to the system resulting from replacing all $\gg$ 's in $N$ by $\geq$. 


$$
\begin{gathered}
\Gamma\left[\vec{b}^{-}, \vec{b}^{+}\right] \vdash_{s a} c: C t(c)\left[\vec{b}_{1}^{+},()\right], \vec{b}_{2}^{+} \\
\text {if } \vec{b}_{1}^{+} \geq \overrightarrow{0}, \vec{b}_{2}^{+} \geq \overrightarrow{1} \\
\left(\Gamma_{1}\left[\vec{b}_{1}^{-}, \vec{b}_{1}^{+}\right],\left(x: t\left[\vec{b}_{2}^{-}, \vec{b}_{2}^{+}\right]\right), \Gamma_{2}\left[\vec{b}_{3}^{-}, \vec{b}_{3}^{+}\right]\right) \vdash_{s a} x: t\left[\vec{b}_{4}^{+}, \vec{b}_{4}^{-}\right],\left(\vec{b}_{5}^{+}, b_{6}^{+}, \vec{b}_{7}^{+}\right) \\
\text {if } \vec{b}_{4}^{+} \geq \vec{b}_{2}^{-}, \vec{b}_{2}^{+} \geq \vec{b}_{4}^{-}, \vec{b}_{5}^{+} \geq 1, b_{6}^{+} \geq 0, \vec{b}_{7}^{+} \geq 1 \\
\frac{\left(\left(x: t_{1}\left[\vec{b}_{1}^{-}, \vec{b}_{1}^{+}\right]\right), \Gamma\left[\vec{b}^{-}, \vec{b}^{+}\right]\right) \vdash_{s a} e: t\left[\vec{b}_{2}^{+}, \vec{b}_{2}^{-}\right],\left(b_{3}^{+}, \vec{b}_{4}^{+}\right)}{\Gamma\left[\vec{b}^{-}, \vec{b}^{+}\right] \vdash_{s a} \lambda x \cdot e: t_{1}\left[\vec{b}_{1}^{-}, \vec{b}_{1}^{+}\right] \rightarrow_{b^{+}} t\left[\vec{b}_{2}^{+}, \vec{b}_{2}^{-}\right], \vec{b}_{4}^{+}} \\
\frac{\Gamma\left[\vec{b}^{-}, \vec{b}^{+}\right] \vdash_{s a} e_{1}: t_{2}\left[\vec{b}_{2}^{-}, \vec{b}_{2}^{+}\right] \rightarrow b_{3}^{+} t_{1}\left[\vec{b}_{4}^{+}, \vec{b}_{4}^{-}\right], \vec{b}_{5}^{+} \text {and } e_{2}: t_{2}\left[\vec{b}_{6}^{+}, \vec{b}_{6}^{-}\right], \vec{b}_{7}^{+}}{\Gamma\left[\vec{b}^{-}, \vec{b}^{+}\right] \vdash_{s a} e_{1} e_{2}: t_{1}\left[\vec{b}_{4}^{+}, \vec{b}_{4}^{-}\right], \vec{b}_{8}^{+}} \\
\text {if } \vec{b}_{6}^{+}=\vec{b}_{2}^{-}, \vec{b}_{2}^{+}=\vec{b}_{6}^{-}, \vec{b}_{8}^{+} \geq \vec{b}_{5}^{+} \sqcap\left(b_{3}^{+} \sqcup \vec{b}_{7}^{+}\right) \\
\frac{\Gamma\left[\vec{b}^{-}, \vec{b}^{+}\right] \vdash_{s a} e_{1}: \text { Bool, } \vec{b}_{3}^{+} \text {and } e_{2}: t\left[\vec{b}_{4}^{+}, \vec{b}_{4}^{-}\right], \vec{b}_{5}^{+} \text {and } e_{3}: t\left[\vec{b}_{6}^{+}, \vec{b}_{6}^{-}\right], \vec{b}_{7}^{+}}{\Gamma \vdash_{s a}\left(\text { if } e_{1} e_{2} e_{3}\right): t\left[\vec{b}_{8}^{+}, \vec{b}_{8}^{-}\right], \vec{b}_{9}^{+}} \\
\text {if } \vec{b}_{8}^{+} \geq \vec{b}_{4}^{+}, \vec{b}_{4}^{-} \geq \vec{b}_{8}^{-}, \vec{b}_{8}^{+} \geq \vec{b}_{6}^{+}, \vec{b}_{6}^{-} \geq \vec{b}_{8}^{-}, \vec{b}_{9}^{+} \geq \vec{b}_{3}^{+} \sqcap\left(\vec{b}_{5}^{+} \sqcup \vec{b}_{7}^{+}\right) \\
\frac{\left(\left(f: t\left[\vec{b}_{1}^{-}, \vec{b}_{1}^{+}\right]\right), \Gamma\left[\vec{b}^{-}, \vec{b}^{+}\right]\right) \vdash_{s a} e: t\left[\vec{b}_{2}^{+}, \vec{b}_{2}^{-}\right],\left(b_{3}^{+}, \vec{b}_{4}^{+}\right)}{\Gamma\left[\vec{b}^{-}, \vec{b}^{+}\right] \vdash_{s a}(\text { rec } f e): t\left[\vec{b}_{5}^{+}, \vec{b}_{5}^{-}\right], \vec{b}_{4}^{+}} \\
\text {if } \vec{b}_{5}^{+} \geq \vec{b}_{2}^{+}, \vec{b}_{2}^{-} \geq \vec{b}_{5}^{-}, \vec{b}_{2}^{+}=\vec{b}_{1}^{-}, \vec{b}_{2}^{-}=\vec{b}_{1}^{+}
\end{gathered}
$$

Figure 6: Annotations on arrows made explicit. 


$$
\begin{gathered}
\Gamma\left[\vec{b}^{-}, \vec{b}^{+}\right] \vdash_{s c} c: C t(c)\left[\vec{b}_{1}^{+},()\right], \vec{b}_{2}^{+}, \\
\left\{\vec{b}_{1}^{+} \geq \overrightarrow{0}, \vec{b}_{2}^{+} \geq \overrightarrow{1}\right\}
\end{gathered}
$$

$$
\begin{gathered}
\left(\Gamma_{1}\left[\vec{b}_{1}^{-}, \vec{b}_{1}^{+}\right],\left(x: t\left[\vec{b}_{2}^{-}, \vec{b}_{2}^{+}\right]\right), \Gamma_{2}\left[\vec{b}_{3}^{-}, \vec{b}_{3}^{+}\right]\right) \vdash_{s a} x: t\left[\vec{b}_{4}^{+}, \vec{b}_{4}^{-}\right],\left(\vec{b}_{5}^{+}, b_{6}^{+}, \vec{b}_{7}^{+}\right), \\
\left\{\left\{\vec{b}_{4}^{+} \geq \vec{b}_{2}^{-}, \vec{b}_{2}^{+} \geq \vec{b}_{4}^{-}, \vec{b}_{5}^{+} \geq 1, b_{6}^{+} \geq 0, \vec{b}_{7}^{+} \geq 1\right\}\right.
\end{gathered}
$$

$$
\frac{\left(\left(x: t_{1}\left[\vec{b}_{1}^{-}, \vec{b}_{1}^{+}\right]\right), \Gamma\left[\vec{b}^{-}, \vec{b}^{+}\right]\right) \vdash_{s a} e: t\left[\vec{b}_{2}^{+}, \vec{b}^{-}\right],\left(b_{3}^{+}, \vec{b}_{4}^{+}\right), C}{\Gamma\left[\vec{b}^{-}, \vec{b}^{+}\right] \vdash_{s a} \lambda x \cdot e: t_{1}\left[\vec{b}_{1}^{-}, \vec{b}_{1}^{+}\right] \rightarrow b_{3}^{+} t\left[\vec{b}_{2}^{+}, \vec{b}_{2}^{-}\right], \vec{b}_{4}^{+}, C}
$$

$$
\frac{\Gamma\left[\vec{b}^{-}, \vec{b}^{+}\right] \vdash_{s a} e_{1}: t_{2}\left[\vec{b}_{2}^{-}, \vec{b}_{2}^{+}\right] \rightarrow b_{3}^{+} t_{1}\left[\vec{b}_{4}^{+}, \vec{b}_{4}^{-}\right], \vec{b}_{5}^{+}, C_{1} \text { and } e_{2}: t_{2}\left[\vec{b}_{b}^{+}, \vec{b}_{6}^{-}\right], \vec{b}_{7}^{+}, C_{2}}{\Gamma\left[\vec{b}^{-}, \vec{b}^{+}\right] \vdash_{s a} e^{+} e_{2}: t_{1}\left[\vec{b}_{+}^{+}, \vec{b}_{4}^{-}\right], \vec{b}_{3}^{+}, C_{1} \cup C_{2} \cup C}
$$

$\Gamma\left[\vec{b}^{-}, \vec{b}^{+}\right] \vdash_{s a} e_{1}:$ Bool, $\vec{b}_{3}^{+}, C_{1}$ and $e_{2}: t\left[\vec{b}_{4}^{+}, \vec{b}_{4}^{-}\right], \vec{b}_{5}^{+}, C_{2}$ and $e_{3}: t\left[\vec{b}_{6}^{+}, \vec{b}_{6}^{-}\right], \vec{b}_{7}^{+}, C_{3}$

$\Gamma\left[\vec{b}^{-}, \vec{b}^{+}\right] \vdash_{s a}\left(\right.$ if $\left.e_{1} e_{2} e_{3}\right): t\left[\vec{b}_{8}^{+}, \vec{b}_{\bar{b}}^{-}\right], \vec{b}_{9}^{+}, C_{1} \cup C_{2} \cup C_{3} \cup C$

where $C=\left\{\vec{b}_{8}^{+} \geq \vec{b}_{4}^{+}, \vec{b}_{4}^{-} \geq \vec{b}_{8}^{-}, \vec{b}_{8}^{+} \geq \vec{b}_{6}^{+}, \vec{b}_{6}^{-} \geq \vec{b}_{8}^{-}, \vec{b}_{9}^{+} \geq \vec{b}_{3}^{+} \cap\left(\vec{b}_{5}^{+} \sqcup \vec{b}_{7}^{+}\right)\right\}$

$$
\begin{gathered}
\frac{\left(\left(f: t\left[\vec{b}_{1}^{-}, \vec{b}_{1}^{+}\right]\right), \Gamma\left[\vec{b}^{-}, \vec{b}^{+}\right]\right) \vdash_{s a} e: t\left[\vec{b}_{2}^{+}, \vec{b}_{2}^{-}\right],\left(b_{3}^{+}, \vec{b}_{4}^{+}\right), C}{\left.\Gamma \vec{b}^{-}, \vec{b}^{+}\right] \vdash_{s a}(\operatorname{rec} f e): t\left[\vec{b}_{5}^{+}, \vec{b}_{5}^{-}\right], \vec{b}_{4}^{+}, C \cup C^{\prime}} \\
\text { where } C^{\prime}=\left\{\vec{b}_{5}^{+} \geq \vec{b}_{2}^{+}, \vec{b}_{2}^{-} \geq \vec{b}_{5}^{-}, \vec{b}_{2}^{+}=\vec{b}_{1}^{-}, \vec{b}_{2}^{-}=\vec{b}_{1}^{+}\right\}
\end{gathered}
$$

Figure 7: An inference system collecting constraints. 
Let $C$ be a constraint system without $\gg$, and let $N$ be a constraint system possibly containing $\gg$. We say that $C \sim N$ iff all strong solutions to $N$ are solutions to $C$, and all solutions to $C$ are weak solutions to $N$.

\section{Normalizing Constraints}

Given an expression $e$, we by means of the system in Fig. 7 are able to produce a proof tree for ()$\vdash_{s a} e: t,(), C$. The strictness variables occurring in $C$ can be divided into two groups: those occurring in $t$, to be denoted $\vec{b}_{1}^{+}$ and $\vec{b}_{1}^{-}$, and those which do not occur in $t$ (but further up in the proof tree), to be denoted $\vec{b}_{0}$ (we do not distinguish between covariant and contravariant here). It turns out that we are able to produce an extended constraint system $N$ with the following property:

- $C \sim N$;

- $N$ is of form $\left\{\vec{b}_{1}^{+} \geq \vec{s}_{1} ; \vec{b}_{0} \gg \vec{s}_{0}\right\}$, with $\vec{s}_{0}$ and $\vec{s}_{1}$ being strictness expressions whose (free) variables belong to $\vec{b}_{1}^{-}$.

Thus each choice of the contravariant positions in $t$ gives rise to a least annotation of the covariant positions (but all greater annotations are also solutions).

Example 4.3 The expression $\lambda f . \lambda x . f(f(x))$ will have type

$$
\left(\text { int } \rightarrow b_{1}^{-} \operatorname{lnt}\right) \rightarrow_{b_{11}^{+}}\left(\text {int } \rightarrow b_{12}^{+} \operatorname{lnt}\right)
$$

with constraint $b_{12}^{+} \geq b_{1}^{-}$. So the minimal types of this expression are $\left(\right.$ int $\left.\rightarrow_{0} \operatorname{lnt}\right) \rightarrow_{0}\left(\right.$ int $\left.\rightarrow_{0} \operatorname{lnt}\right)$ and $\left(\right.$ int $\left.\rightarrow_{1} \operatorname{lnt}\right) \rightarrow_{0}\left(\right.$ int $\left.\rightarrow_{1} \operatorname{lnt}\right)$.

The reason for also being interested in the value of $\vec{b}_{0}$ is that the strictness types of the subexpressions may be useful, e.g. to produce the translation in Sect. 5. We should of course use the least such value.

Example 4.4 Consider the expression $e_{1} e_{2}$, where $e_{1}=\lambda f . \lambda x . f(f(x))$ and $e_{2}=(\lambda y . y) . e_{1}$ has type $\left(\right.$ int $\rightarrow b_{01}^{-}$Int $) \rightarrow b_{01}^{+}\left(\right.$int $\rightarrow b_{1}^{+}$Int $), e_{2}$ has type int $\rightarrow b_{02}^{+}$Int 
and $e_{1} e_{2}$ has type int $\rightarrow b_{1}^{+} \operatorname{lnt}$ ), where the constraints look like $b_{02}^{+}=b_{01}^{-}$and $b_{1}^{+} \geq b_{01}^{-}$This can be normalized into $b_{1}^{+} \geq 0, b_{02}^{+} \gg 0$ and $b_{01}^{-} \gg 0$. So one should assign $e_{1}$ type $\left(\right.$ int $\left.\rightarrow_{0} \operatorname{lnt}\right) \rightarrow_{0}\left(\right.$ int $\rightarrow_{0}$ Int $)$, that is assume that $f$ has type int $\rightarrow_{0}$ Int.

In order to achieve our goal we for each rule in Fig. 7 of form

$$
\frac{\ldots \Gamma_{i} \vdash_{s a} e_{i}: t_{i}, W_{i}, C_{i} \ldots}{\Gamma \vdash_{s a} e: t, W, C_{1} \cup \ldots \cup C_{n} \cup C}
$$

must proceed as follows: assume that there for each $i$ exists an extended constraint system $N_{i}$ such that

- $C_{i} \sim N_{i}$

- $N_{i}$ is of form $\left\{\vec{b}_{i 1}^{+} \geq \vec{s}_{i 1}, \vec{b}_{i 0} \gg \vec{s}_{i 0}\right\}$ where $\left(\vec{b}_{i 1}^{+}, \vec{b}_{i 1}^{-}\right)$are the strictness variables occurring in $\Gamma_{i}, t_{i}$ or $W_{i}$, where $\vec{b}_{i 0}$ are the remaining strictness variables in $C_{i}$, and where the free variables of the strictness expressions $\vec{s}_{i 1}$ and $\vec{s}_{i 0}$ belong to $\vec{b}_{i 1}^{-}$.

Then we must be able to construct $N$ (i.e. do a normalization) such that

- $C_{1} \cup \cdots C_{n} \cup C \sim N$;

- $N$ is of form $\left\{\vec{b}_{1}^{+} \geq \vec{s}_{1}, \vec{b}_{0} \gg \vec{s}_{0}\right\}$ where $\left(\vec{b}_{1}^{+}, \vec{b}_{1}^{-}\right)$are the strictness variables occurring in $\Gamma, t$ or $W$, where $\vec{b}_{0}$ are the remaining strictness variables in $C$, and where the free variables of the strictness expressions $\vec{s}_{1}$ and $\vec{s}_{0}$ belong to $\vec{b}_{1}^{-}$.

Before embarking on the normalization process, it will be convenient to describe some of the tools to be used.

\section{Some Transformation Rules}

Let $N$ and $N^{\prime}$ be extended constraint systems. We say that it is correct to transform $N$ into $N^{\prime}$ if all strong solutions to $N^{\prime}$ also are strong solutions 
to $N$, and if all weak solutions to $N$ also are weak solutions to $N^{\prime}$. If it is correct to transform $N$ into $N^{\prime}$, we clearly have that if $C \sim N$ then also $C \sim N^{\prime}$. We now list some correct transformations:

Fact 4.5 Suppose $N$ contains the constraints $\vec{b} \geq \vec{s}_{1}, \vec{b} \geq \vec{s}_{2}$. Then these can be replaced by the constraint $\vec{b} \geq \vec{s}_{1} \sqcup \vec{s}_{2}$.

Fact 4.6 Suppose $N$ contains the constraint $\vec{b} \geq \vec{s}$ or the constraint $\vec{b}=\vec{s}$. Then this can be replaced by $\vec{b} \gg \vec{s}$.

Fact 4.7 Suppose $N$ contains the constraints $\vec{b}_{1} \geq g_{1}\left(\vec{b}_{2}\right)$ and $\vec{b}_{2} \gg g_{2}\left(\vec{b}_{3}\right)$. Then the former constraint can be replaced by the constraint $\vec{b}_{1} \geq g_{1}\left(g_{2}\left(\vec{b}_{3}\right)\right)$, yielding a new constraint system $N^{\prime}$.

(In other words, if we have the constraints $\vec{b} \geq \vec{s}$ and $\vec{b}_{i} \gg \vec{s}_{i}$ it is safe to replace the former constraint by $\vec{b} \geq \vec{s}\left[\vec{s}_{i} / \vec{b}_{i}\right]$.)

Proof: Let a strong solution to $N^{\prime}$ be given. Wrt. this solution, we have $\vec{b}_{2}=g_{2}\left(\vec{b}_{3}\right), \vec{b}_{1} \geq g_{1}\left(g_{2}\left(\vec{b}_{3}\right)\right)$ and hence also $\vec{b}_{1} \geq g_{1}\left(\vec{b}_{2}\right)-$ thus this solution is also a strong solution to $N$.

Now let a weak solution to $N$ be given. Wrt. this solution, we have $\vec{b}_{1} \geq g_{1}\left(\vec{b}_{2}\right)$ and $\vec{b}_{2} \geq g_{2}\left(\vec{b}_{3}\right)$. Due to the monotonicity of $g_{1}$, we also have $\vec{b}_{1} \geq g_{1}\left(g_{2}\left(\vec{b}_{3}\right)\right)$ showing that this solution is also a weak solution to $N^{\prime}$.

Fact 4.8 Suppose $N$ contains the constraints $\vec{b}_{1} \gg g_{1}\left(\vec{b}_{2}\right)$ and $\vec{b}_{2} \gg g_{2}\left(\vec{b}_{3}\right)$. Then the former constraint can be replaced by the constraint $\vec{b}_{1} \gg g_{1}\left(g_{2}\left(\vec{b}_{3}\right)\right)$, yielding a new constraint system $N^{\prime}$.

(In other words, if we have the constraints $\vec{b} \gg \vec{s}$ and $\vec{b}_{i} \gg \vec{s}_{i}$ it is safe to replace the former constraint by $\vec{b} \gg \vec{s}\left[\vec{s}_{i} / \vec{b}_{i}\right]$.)

Proof: Let a strong solution to $N^{\prime}$ be given. Wrt. this solution, we have $\vec{b}_{2}=g_{2}\left(\vec{b}_{3}\right), \vec{b}_{1}=g_{1}\left(g_{2}\left(\vec{b}_{3}\right)\right)$ and hence also $\vec{b}_{1}=g_{1}\left(\vec{b}_{2}\right)-$ thus this solution is also a strong solution to $N$.

Now let a weak solution to $N$ be given. Wrt. this solution, we have $\vec{b}_{1} \geq g_{1}\left(\vec{b}_{2}\right)$ and $\vec{b}_{2} \geq g_{2}\left(\vec{b}_{3}\right)$. Due to the monotonicity of $g_{1}$, we also have $\vec{b}_{1} \geq g_{1}\left(g_{2}\left(\vec{b}_{3}\right)\right)$ showing that this solution is also a weak solution to $N^{\prime}$. 
Fact 4.9 Suppose $N$ contains the constraint $\vec{b}_{1} \geq g\left(\vec{b}_{1}, \vec{b}_{2}\right)$. Then this can be replaced by the constraint $\vec{b}_{1} \gg g^{\prime}\left(\vec{b}_{2}\right)$ (yieldig $N^{\prime}$ ), where

$$
g^{\prime}\left(\vec{b}_{2}\right)=\sqcup_{k} h^{k}(\overrightarrow{0}) \text { with } h(\vec{b})=g\left(\vec{b}, \vec{b}_{2}\right)
$$

(this just amounts to Tarski's theorem - notice that it will actually suffice with $\left|\vec{b}_{1}\right|$ iterations).

Proof: $\quad$ First suppose that we have a strong solution to $N^{\prime}$, i.e. $\vec{b}_{1}=$ $g^{\prime}\left(\vec{b}_{2}\right)$. Since $\vec{b}_{1}=\sqcup_{k} h^{k}(\overrightarrow{0})$, standard reasoning on the monotone and hence (as finite lattices) continuous function $h$ tells us that $\vec{b}_{1}=h\left(\vec{b}_{1}\right)$, i.e. $\vec{b}_{1}=$ $g\left(\vec{b}_{1}, \vec{b}_{2}\right)$. This shows that we have a strong solution to $N$.

Now suppose that we have a weak solution to $N$, i.e. $\vec{b}_{1} \geq g\left(\vec{b}_{1}, \vec{b}_{2}\right)$. In order to show that this also is a weak solution to $N^{\prime}$, we must show that $\vec{b}_{1} \geq g^{\prime}\left(\vec{b}_{2}\right)$. This can be done by showing that $\vec{b}_{1} \geq \vec{b}$ implies $\vec{b}_{1} \geq h(\vec{b})$. But if $\vec{b}_{1} \geq \vec{b}$ we have $\vec{b}_{1} \geq g\left(\vec{b}_{1}, \vec{b}_{2}\right) \geq g\left(\vec{b}, \vec{b}_{2}\right)=h(\vec{b})$.

It is easy to see that $g^{\prime}$ is monotone.

\subsection{The Normalization Process}

We shall examine the various constructs: for constants and variables the normalization process is trivial, as the constraints generated are of the required form. Neither does the rule for abstractions cause any troubles, since no new constraints are generated and since a strictness variable appears in the premise of the rule iff it appears in the conclusion (and with the same polarity). Now let us focus upon the remaining constructs.

\section{Normalizing the Rule for Application}

Recall the rule

$$
\frac{\Gamma\left[\vec{b}^{-}, \vec{b}^{+}\right] \vdash_{s a} e_{1} t_{2}\left[\vec{b}_{2}^{-}, \vec{b}_{2}^{+}\right] \rightarrow_{b_{3}^{+}} t_{1}\left[\vec{b}_{4}^{+}, \vec{b}_{4}^{-}\right], \vec{b}_{5}^{+}, C_{1} \text { and } e_{2}: t_{2}\left[\vec{b}_{6}^{+}, \vec{b}_{6}^{-}\right], \vec{b}_{7}^{+}, C_{2}}{\Gamma\left[\vec{b}^{-}, \vec{b}^{+}\right] \vdash_{s a} e_{1} e_{2}: t_{1}\left[\vec{b}_{4}^{+}, \vec{b}_{4}^{-}\right], \vec{b}_{8}^{+}, C_{1} \cup C_{2} \cup C}
$$


where $C=\left\{\vec{b}_{6}^{+}=\vec{b}_{2}^{-}, \vec{b}_{2}^{+}=\vec{b}_{6}^{-}, \vec{b}_{8}^{+} \geq \vec{b}_{5}^{+} \sqcap\left(\vec{b}_{3}^{+} \sqcup \vec{b}_{7}^{+}\right)\right\}$.

Let $\vec{b}_{0}$ be the "extra" strictness variables of $C_{1}$ and $\vec{b}_{1}$ the extra strictness variables of $C_{2}$. Then we are entitled to assume that there exist $N_{1}, N_{2}$ with $C_{1} \sim N_{1}, C_{2} \sim N_{2}$ such that $N_{1}$ takes the form

$$
\vec{b}^{+} \geq \vec{s}_{a} \quad \vec{b}_{2}^{+} \geq \vec{s}_{2} \quad b_{3}^{+} \geq s_{3} \quad \vec{b}_{4}^{+} \geq \vec{s}_{4} \quad \vec{b}_{5}^{+} \geq \vec{s}_{5} \quad \vec{b}_{0} \gg \vec{s}_{0}
$$

(where the free variables of the strictness expressions above belong to $\left\{\vec{b}^{-}, \vec{b}_{2}^{-}\right.$, $\left.\vec{b}_{4}^{-}\right\}$) and such that $N_{2}$ takes the form

$$
\vec{b}^{+} \geq \vec{s}_{b} \quad \vec{b}_{6}^{+} \geq \vec{s}_{6} \quad \vec{b}_{7}^{+} \geq \vec{s}_{7} \quad \vec{b}_{1} \gg \vec{s}_{1}
$$

(where the free variables of the strictness expressions above belong to $\left\{\vec{b}^{-}\right.$, $\left.\vec{b}_{6}^{-}\right\}$.)

Clearly $C_{1} \cup C_{2} \cup C \sim N_{1} \cup N_{2} \cup C$. We shall now manipulate $N_{1} \cup N_{2} \cup C$ (by means of correct transformations) with the aim of getting something of the desired form.

The first step is to use Fact 4.5 to replace the two inequalities for $\vec{b}^{+}$by one, and at the same time exploit that $\vec{b}_{2}^{+}=\vec{b}_{6}^{-}$and $\vec{b}_{6}^{+}=\vec{b}_{2}^{-}$. As a result, we arrive at

$$
\begin{array}{llll}
\vec{b}^{+} \geq \vec{s}_{a} \sqcup \vec{s}_{b} & \vec{b}_{6}^{-} \geq \vec{s}_{2} & \vec{b}_{3}^{+} \geq s_{3} & \vec{b}_{4}^{+} \geq \vec{s}_{4} \\
\vec{b}_{5}^{+} \geq \vec{s}_{5} & \vec{b}_{0} \gg \vec{s}_{0} & \vec{b}_{2}^{-} \geq \vec{s}_{6} & \vec{b}_{7}^{+} \geq \vec{s}_{7} \\
\vec{b}_{1} \gg \vec{s}_{1} & \vec{b}_{6}^{+}=\vec{b}_{2}^{-} & \vec{b}_{2}^{+}=\vec{b}_{6}^{-} & \vec{b}_{8}^{+} \geq \vec{b}_{5}^{+} \sqcap\left(b_{3}^{+} \sqcup \vec{b}_{7}^{+}\right)
\end{array}
$$

We now focus upon the pair of constraints

$$
\left(\vec{b}_{6}^{-}, \vec{b}_{2}^{+}\right) \geq\left(\vec{s}_{2}, \vec{s}_{6}\right)
$$

According to Fact 4.9, these can be replaced by the constraints

$$
\left(\vec{b}_{6}^{-}, \vec{b}_{2}^{+}\right) \gg\left(\vec{S}_{2}, \vec{S}_{6}\right)
$$


where $\left(\vec{S}_{2}, \vec{S}_{6}\right)$ is given as the "limit" of the chain with elements $\left(\vec{s}_{2 n}, \vec{s}_{6 n}\right)$ given by

$$
\begin{aligned}
\left(\vec{s}_{20}, \vec{s}_{60}\right) & =\overrightarrow{0} \\
\left(\vec{s}_{2(n+1)}, \vec{s}_{6(n+1)}\right) & =\left(\vec{s}_{2}, \vec{s}_{6}\right)\left[\left(\vec{s}_{2 n}, \vec{s}_{6 n}\right) /\left(\vec{b}_{6}^{-}, \vec{b}_{2}^{-}\right)\right]
\end{aligned}
$$

This limit can be found as the $k^{\prime}$ th element, where $k=\left|\left(\vec{b}_{2}^{-}, \vec{b}_{6}^{-}\right)\right|$.

As $\vec{s}_{2}$ does not contain $\vec{b}_{6}^{-}$and $\vec{s}_{6}$ does not contain $\vec{b}_{2}^{-}$, the above can be simplified into

$$
\begin{array}{ll}
\vec{s}_{20}=\overrightarrow{0} & \vec{s}_{2(n+1)}=\vec{s}_{2}\left[\vec{s}_{6 n} / \vec{b}_{2}^{-}\right] \\
\vec{s}_{20}=\overrightarrow{0} & \vec{s}_{6(n+1)}=\vec{s}_{6}\left[\vec{s}_{2 n} / \vec{b}_{6}^{-}\right]
\end{array}
$$

Our next step is to substitute in the new constraints for $\vec{b}_{2}^{-}$and $\vec{b}_{6}^{-}$, using Fact 4.7 and Fact 4.8. We arrive at (after also having used Fact 4.6)

$$
\begin{array}{lll}
\vec{b}^{+} \geq \vec{s}_{a}\left[\vec{S}_{6} / \vec{b}_{2}^{-}\right] \sqcup \vec{s}_{b}\left[\vec{S}_{2} / \vec{b}_{6}^{-}\right] & \vec{b}_{6}^{-} \gg \vec{S}_{2} & \vec{b}_{3}^{+} \gg s_{3}\left[\vec{S}_{6} / \vec{b}_{2}^{-}\right] \\
\vec{b}_{4}^{+} \geq \vec{s}_{4}\left[\vec{S}_{6} / \vec{b}_{2}^{-}\right] & \vec{b}_{5}^{+} \gg \vec{s}_{5}\left[\vec{S}_{6} / \vec{b}_{2}^{-}\right] & \vec{b}_{0} \gg \vec{s}_{0}\left[\vec{S}_{6} / \vec{b}_{2}^{-}\right] \\
\vec{b}_{2}^{-} \gg \vec{S}_{6} & \vec{b}_{7}^{+} \gg \vec{s}_{7}\left[\vec{S}_{2} / \vec{b}_{6}^{-}\right] & \vec{b}_{1} \gg \vec{s}_{1}\left[\vec{S}_{2} / \vec{b}_{6}^{-}\right] \\
\vec{b}_{6}^{+} \gg \vec{S}_{6} & \vec{b}_{2}^{+} \gg \vec{S}_{2} & \vec{b}_{8}^{+} \geq \vec{b}_{5}^{+} \sqcap\left(\vec{b}_{3}^{+} \sqcup \vec{b}_{7}^{+}\right)
\end{array}
$$

Finally we use Fact 4.7 on the constraint for $\vec{b}_{8}^{+}$, arriving at

$$
\begin{array}{lll}
\vec{b}^{+} \geq \vec{s}_{a}\left[\vec{S}_{6} / \vec{b}_{2}^{-}\right] \sqcup \vec{s}_{b}\left[\vec{S}_{2} / \vec{b}_{6}^{-}\right] & \vec{b}_{6}^{-} \gg \vec{S}_{2} & b_{3}^{+} \gg s_{3}\left[\vec{S}_{6} / \vec{b}_{2}^{-}\right] \\
b_{4}^{+} \geq \vec{s}_{4}\left[\vec{S}_{6} / \vec{b}_{2}^{-}\right] & \vec{b}_{5}^{+} \gg \vec{s}_{5}\left[\vec{S}_{6} / \vec{b}_{2}^{-}\right] & \vec{b}_{0} \gg \vec{s}_{0}\left[\vec{S}_{6} / \vec{b}_{2}^{-}\right] \\
\vec{b}_{2}^{-} \gg \vec{S}_{6} & \vec{b}_{7}^{+} \gg \vec{s}_{7}\left[\vec{S}_{2} / \vec{b}_{6}^{-}\right] & \vec{b}_{1} \gg \vec{s}_{1}\left[\vec{S}_{6} / \vec{b}_{2}^{-}\right] \\
\vec{b}_{2}^{-} \gg \vec{S}_{6} & \vec{b}_{2}^{+} \gg \vec{S}_{2} & \\
\vec{b}_{8}^{+} \geq \vec{s}_{5}\left[\vec{S}_{6} / \vec{b}_{2}^{-}\right] \sqcap\left(s_{3}\left[\vec{S}_{2} / \vec{b}_{6}^{-}\right] \sqcup \vec{s}_{7}\left[\vec{S}_{6} / \vec{b}_{2}^{-}\right]\right) & &
\end{array}
$$

This is of the desired form, as it is quite easy to check that the only strictness variables occurring in the expressions on the right hand sides are those occurring in $\vec{b}^{-}$and in $\vec{b}_{4}^{-}$.

\section{Normalizing the Rule for Conditionals}

Recall the rule 
$\frac{\Gamma\left[\vec{b}^{-}, \vec{b}^{+}\right] \vdash_{s a} e_{1}: \text { Bool, } \vec{b}_{3}^{+}, C_{1} \text { and } e_{2}: t\left[\vec{b}_{4}^{+}, \vec{b}_{4}^{-}\right], \vec{b}_{5}^{+}, C_{2} \text { and } e_{3}: t\left[\vec{b}_{6}^{-}, \vec{b}_{6}^{-}\right], \vec{b}_{7}^{+}, C_{3}}{\Gamma\left[\vec{b}^{-}, \vec{b}^{+}\right] \vdash_{s a} \text { if } e_{1} e_{2} e_{3}: t\left[\vec{b}_{8}^{+}, \vec{b}_{8}^{-}\right], \vec{b}_{9}^{+}, C_{1} \cup C_{2} \cup C_{3} \cup C}$

where $C=\left\{\vec{b}_{8}^{+} \geq \vec{b}_{4}^{+}, \vec{b}_{8}^{+} \geq \vec{b}_{6}^{+}, \vec{b}_{4}^{-} \geq \vec{b}_{8}^{-}, \vec{b}_{6}^{-} \geq \vec{b}_{8}^{-}, \vec{b}_{9}^{+} \geq \vec{b}_{3}^{+} \sqcap\left(\vec{b}_{5}^{+} \sqcup \vec{b}_{7}^{+}\right)\right\}$.

Let $\vec{b}_{0}$ be the "extra" strictness variables of $C_{1}, \vec{b}_{1}$ the extra strictness variables of $C_{2}$ and $\vec{b}_{2}$ the extra strictness variables of $C_{3}$. Then we are entitled to assume that there exist $N_{1}, N_{2}, N_{3}$ with $C_{1} \sim N_{1}, C_{2} \sim N_{2}, C_{3} \sim$ $N_{3}$ such that $N_{1}$ takes the form

$$
\vec{b}^{+} \geq \vec{s}_{a} \quad \vec{b}_{3}^{+} \geq \vec{s}_{3} \quad \vec{b}_{0} \gg \vec{s}_{0}
$$

(where the free variables of the strictness expressions above belong to $\vec{b}^{-}$) and such that $N_{2}$ takes the form

$$
\vec{b}^{+} \geq \vec{s}_{b} \quad \vec{b}_{4}^{+} \geq \vec{s}_{4} \quad \vec{b}_{5}^{+} \geq \vec{s}_{5} \quad \vec{b}_{1} \gg \vec{s}_{1}
$$

(where the free variables of the strictness expressions above belong to $\left(\vec{b}^{-}\right.$, $\left.\vec{b}_{4}^{-}\right)$) and such that $N_{3}$ takes the form

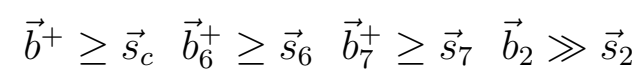

(where the free variables of the strictness expressions above belong to $\left(\vec{b}^{-}\right.$, $\left.\vec{b}_{6}^{-}\right)$).

Clearly $C_{1} \cup C_{2} \cup C_{3} \cup C \sim N_{1} \cup N_{2} \cup N_{3} \cup C$. we shall now manipulate $N_{1} \cup N_{2} \cup N_{3} \cup C$ (by means of correct transformations) with the aim of getting something of the desired form.

The first step is to use Fact 4.6 (i.e. replace $\geq$ by $\gg$ ) on the inequalities $\vec{b}_{4}^{-} \geq \vec{b}_{8}^{-}$and $\vec{b}_{6}^{-} \geq \vec{b}_{8}^{-}$, afterwards use Fact 4.7 and Fact 4.8 to substitute $\vec{b}_{4}^{-}$ $\left(\vec{b}_{6}^{-}\right)$by $\vec{b}_{8}^{-}$. By also using Fact 4.5 to replace the two inequalities for $\vec{b}_{8}^{+}$by one and to replace the three inequalities for $\vec{b}^{+}$by one, we arrive at 


$$
\begin{array}{lll}
\vec{b}^{+} \geq \vec{s}_{a} \sqcup \vec{s}_{b}\left[\vec{b}_{8}^{-} / \vec{b}_{4}^{-}\right] \sqcup \vec{s}_{c}\left[\vec{b}_{8}^{-} / \vec{b}_{6}^{-}\right] & \vec{b}_{3}^{+} \geq \vec{s}_{3} & \vec{b}_{0} \gg \vec{s}_{0} \\
\vec{b}_{4}^{+} \geq \vec{s}_{4}\left[\vec{b}_{8}^{-} / \vec{b}_{4}^{-}\right] & \vec{b}_{5}^{+} \geq \vec{s}_{5}\left[\vec{b}_{8}^{-} / \vec{b}_{4}^{-}\right] & \vec{b}_{1} \gg \vec{s}_{1}\left[\vec{b}_{8}^{-} / \vec{b}_{4}^{-}\right] \\
\vec{b}_{6}^{+} \geq \vec{s}_{6}\left[\vec{b}_{8}^{-} / \vec{b}_{6}^{-}\right] & \vec{b}_{7}^{+} \geq \vec{s}_{7}\left[\vec{b}_{8}^{-} / \vec{b}_{6}^{-}\right] & \vec{b}_{2} \gg \vec{s}_{2}\left[\vec{b}_{8}^{-} / \vec{b}_{6}^{-}\right] \\
\vec{b}_{8}^{+} \geq \vec{b}_{4}^{+} \sqcup \vec{b}_{6}^{+} & \vec{b}_{4}^{-} \gg \vec{b}_{8}^{-} & \vec{b}_{6}^{-} \gg \vec{b}_{8}^{-} \\
\vec{b}_{9}^{+} \geq \vec{b}_{3}^{+} \sqcap\left(\vec{b}_{5}^{+} \sqcup \vec{b}_{7}^{+}\right) & &
\end{array}
$$

Next we replace some $\geq$ by $\gg$ (Fact 4.6 ), enabling us to use Fact 4.7 on the constraints for $\vec{b}_{8}^{+}$and $\vec{b}_{9}^{+}$. We arrive at

$$
\begin{array}{lll}
\vec{b}^{+} \geq \vec{s}_{a} \sqcup \vec{s}_{b}\left[\vec{b}_{8}^{-} / \vec{b}_{4}^{-}\right] \sqcup \vec{s}_{c}\left[\vec{b}_{8}^{-} / \vec{b}_{6}^{-}\right] & \vec{b}_{3}^{+} \gg \vec{s}_{3} & \vec{b}_{0} \gg \vec{s}_{0} \\
\vec{b}_{4}^{+} \gg \vec{s}_{4}\left[\vec{b}_{8}^{-} / \vec{b}_{4}^{-}\right] & \vec{b}_{5}^{+} \gg \vec{s}_{5}\left[\vec{b}_{8}^{-} / \vec{b}_{4}^{-}\right] & \vec{b}_{1} \gg \vec{s}_{1}\left[\vec{b}_{8}^{-} / \vec{b}_{4}^{-}\right] \\
\vec{b}_{6}^{+} \gg \vec{s}_{6}\left[\vec{b}_{8}^{-} / \vec{b}_{6}^{-}\right] & \vec{b}_{7}^{+} \gg \vec{s}_{7}\left[\vec{b}_{8}^{-} / \vec{b}_{6}^{-}\right] & \vec{b}_{2} \gg \vec{s}_{2}\left[\vec{b}_{8}^{-} / \vec{b}_{6}^{-}\right] \\
\vec{b}_{8}^{+} \geq \vec{s}_{4}\left[\vec{b}_{8}^{-} / \vec{b}_{4}^{-}\right] \sqcup\left[\vec{b}_{8}^{-} / \vec{b}_{6}^{-}\right] & \vec{b}_{4}^{-} \gg \vec{b}_{8}^{-} & \vec{b}_{6}^{-} \gg \vec{b}_{8}^{-} \\
\vec{b}_{9}^{+} \geq \vec{s}_{3} \sqcap\left(\vec{s}_{5}\left[\vec{b}_{8}^{-} / \vec{b}_{4}^{-}\right] \sqcap \vec{s}_{7}\left[\vec{b}_{8}^{-} / \vec{b}_{6}^{-}\right]\right) & &
\end{array}
$$

This is of the desired form, as it is quite easy to check that the only strictness variables occurring in the expressions on the right hand sides are those occurring in $\vec{b}^{-}$and in $\vec{b}_{8}^{-}$.

\section{Normalizing the Rule for Recursion}

Recall the rule

$$
\frac{\left(\left(f: t\left[\vec{b}_{1}^{-}, \vec{b}_{1}^{+}\right]\right), \Gamma\left[\vec{b}^{-}, \vec{b}^{+}\right]\right) \vdash_{s a} e: t\left[\vec{b}_{2}^{+}, \vec{b}_{2}^{-}\right],\left(b_{3}^{+}, \vec{b}_{4}^{+}\right), C}{\Gamma\left[\vec{b}^{-}, \vec{b}^{+}\right] \vdash_{s a} \text { rec } f e: t\left[\vec{b}_{5}^{+}, \vec{b}_{5}^{-}\right], \vec{b}_{4}^{+}, C \cup C^{\prime}}
$$

where $C^{\prime}=\left\{\vec{b}_{1}^{-}=\vec{b}_{2}^{+}, \vec{b}_{2}^{-}=\vec{b}_{1}^{+}, \vec{b}_{5}^{+} \geq \vec{b}_{2}^{+}, \vec{b}_{2}^{-} \geq \vec{b}_{5}^{-}\right\}$.

Let $b_{0}$ be the "extra" strictness variables of $C$. We are entitled to assume that there exist $N$ with $C \sim N$ such that $N$ takes the form

$$
\vec{b}^{+} \geq \vec{s} \quad \vec{b}_{1}^{+} \geq \vec{s}_{1} \quad \vec{b}_{2}^{+} \geq \vec{s}_{2} \quad \vec{b}_{3}^{+} \geq \vec{s}_{3} \quad \vec{b}_{4}^{+} \geq \vec{s}_{4} \quad \vec{b}_{0} \gg \vec{s}_{0}
$$

(where the free variables of the strictness expressions above belong to $\left(\vec{b}^{-}, \vec{b}_{1}^{-}\right.$, $\left.\vec{b}_{2}^{-}\right)$.) 
Clearly $C \cup C^{\prime} \sim N \cup C^{\prime}$. We shall now manipulate $N \cup C^{\prime}$ (by means of correct transformations) with the aim of getting something of the desired form.

The first step is to exploit that $\vec{b}_{2}^{-}=\vec{b}_{1}^{+}$and $\vec{b}_{1}^{-}=\vec{b}_{2}^{+}$, and afterwards exploit Fact 4.5 to replace the two inequalities for $\vec{b}_{2}^{-}$by one. We arrive at

$$
\begin{array}{lll}
\vec{b}^{+} \geq \vec{s} & \vec{b}_{2}^{-} \geq \vec{s}_{1} \sqcup \vec{b}_{5}^{-} & \vec{b}_{1}^{-} \geq \vec{s}_{2} \\
b_{3}^{+} \geq \vec{s}_{3} & \vec{b}_{4}^{+} \geq \vec{s}_{4} & \vec{b}_{0} \gg \vec{s}_{0} \\
\vec{b}_{1}^{-}=\vec{b}_{2}^{+} & \vec{b}_{2}^{-}=\vec{b}_{1}^{+} & \vec{b}_{5}^{+} \geq \vec{b}_{2}^{+}
\end{array}
$$

We now focus upon the pair of constraints

$$
\left(\vec{b}_{2}^{-}, \vec{b}_{1}^{-}\right) \geq\left(\vec{s}_{1} \sqcup \vec{b}_{5}^{-}, \vec{s}_{2}\right)
$$

According to Fact 4.9, these can be replaced by the constraints

$$
\left(\vec{b}_{2}^{-}, \vec{b}_{1}^{-}\right) \gg\left(\vec{S}_{1}, \vec{S}_{2}\right)
$$

where $\left(\vec{S}_{1}, \vec{S}_{2}\right)$ is given as the "limit" of the chain with elements $\left(\vec{s}_{1 n}, \vec{s}_{2 n}\right)$ given by

$$
\begin{aligned}
\left(\vec{s}_{10}, \vec{s}_{20}\right) & =\overrightarrow{0} \\
\left(\vec{s}_{1(n+1)}, \vec{s}_{2(n+1)}\right) & =\left(\vec{s}_{2} \sqcup \vec{b}_{5}^{-}, \vec{s}_{2}\right)\left[\left(\vec{s}_{1 n}, \vec{s}_{2 n}\right) /\left(\vec{b}_{2}^{-}, \vec{b}_{1}^{-}\right)\right]
\end{aligned}
$$

This limit can be found as the $k^{\prime}$ th element, where $k=\left|\left(\vec{b}_{1}^{-}, \vec{b}_{2}^{-}\right)\right|$.

Our next step is to substitute in the new constraints for $\vec{b}_{1}^{-}$and $\vec{b}_{2}^{-}$, using Fact 4.7 and Fact 4.8. We arrive at (after also having used Fact 4.6 to replace $=$ by $\gg)$

$$
\begin{array}{lll}
\vec{b}^{+} \geq \vec{s}\left[\left(\vec{S}_{1}, \vec{S}_{2}\right) /\left(\vec{b}_{2}^{-}, \vec{b}_{1}^{-}\right)\right] & \vec{b}_{2}^{-} \gg \vec{S}_{1} & \vec{b}_{1}^{-} \gg \vec{S}_{2} \\
\vec{b}_{3}^{+} \geq \vec{s}_{3}\left[\left(\vec{S}_{1}, \vec{S}_{2}\right) /\left(\vec{b}_{2}^{-}, \vec{b}_{1}^{-}\right)\right] & \vec{b}_{4}^{+} \geq \vec{s}_{4}\left[\left(\vec{S}_{1}, \vec{S}_{2}\right) /\left(\vec{b}_{2}^{-}, \vec{b}_{1}^{-}\right)\right] & \vec{b}_{0} \gg \vec{s}_{0}\left[\left(\vec{S}_{1}, \vec{S}_{2}\right) /\left(\vec{b}_{2}^{-}, \vec{b}_{1}^{-}\right)\right] \\
\vec{b}_{2}^{+} \gg \vec{b}_{1}^{-} & \vec{b}_{1}^{+} \gg \vec{b}_{2}^{-} & \vec{b}_{5}^{+} \geq \vec{b}_{2}^{+}
\end{array}
$$

Finally we use Fact 4.8 on the inequalities for $\vec{b}_{2}^{+}$and $\vec{b}_{1}^{+}$, and subsequently use Fact 4.7 on the inequality for $\vec{b}_{5}^{+}$. At the same time we replace some $\geq$ by $\gg$, and arrive at 


$$
\begin{array}{lll}
\vec{b}^{+} \geq \vec{s}\left[\left(\vec{S}_{1}, \vec{S}_{2}\right) /\left(\vec{b}_{2}^{-}, \vec{b}_{1}^{-}\right)\right] & \vec{b}_{2}^{-} \gg \vec{S}_{1} & \vec{b}_{1}^{-} \gg \vec{S}_{2} \\
\vec{b}_{3}^{+} \gg \vec{s}_{3}\left[\left(\vec{S}_{1}, \vec{S}_{2}\right) /\left(\vec{b}_{2}^{-}, \vec{b}_{1}^{-}\right)\right] & \vec{b}_{4}^{+} \geq \vec{s}_{4}\left[\left(\vec{S}_{1}, \vec{S}_{2}\right) /\left(\vec{b}_{2}^{-}, \vec{b}_{1}^{-}\right)\right] & \vec{b}_{0} \gg \vec{s}_{0}\left[\left(\vec{S}_{1}, \vec{S}_{2}\right) /\left(\vec{b}_{2}^{-}, \vec{b}_{1}^{-}\right)\right] \\
\vec{b}_{2}^{+} \gg \vec{S}_{2} & \vec{b}_{1}^{+} \gg \vec{S}_{1} & \vec{b}_{5}^{+} \geq \vec{S}_{2}
\end{array}
$$

This is of the desired form, as it is quite easy to check that the only strictness variables occurring in the expressions on the right hand sides are those occurring in $\vec{b}^{-}$and in $\vec{b}_{5}^{-}$.

\subsection{Complexity Issues}

One would of course like to estimate the complexity of the algorithm just developed. Before doing so, however, several issues have to be clarified, e.g.

- what should the input size parameter be? Natural choices could be the size of the expression, the size of its type, the maximal size of a subexpressions type, etc;

- how do we represent strictness expressions, and how do we perform the various manipulations on strictness expressions?

To give an in-depth treatment of these matters is beyond the scope of this paper, and hence we refrain from giving a complexity analysis...

\section{Tanslating from CBN to CBV}

In this section we give an example of the use of strictness annotations. We shall consider the following problem: given a $\lambda$-expression $e$ annotated with strictness types, find a $\lambda$-expression $e^{\prime}$ such that $e$ when evaluated using CBN yields the same result as $e^{\prime}$ when evaluated using CBV. The development of the translation algorithm will proceed in a number of steps. 


\section{The Mapping $Z$ from $\mathcal{T}_{s a}$ into $\mathcal{T}$}

If $e$ can be assigned strictness type $t$, it (cf. Fact 3.3) has underlying type $\mathrm{E}(t)$. We cannot expect $e^{\prime}$, the translation of $e$ into $\mathrm{CBV}$, to have underlying type $\mathrm{E}(t)$ - rather $e^{\prime}$ should have type $Z(t)$, where $Z$ besides removing annotations from arrows also thunkifies arguments to non-strict functions. That is, we have $Z($ Base $)=$ Base, $Z\left(t_{1} \rightarrow_{0} t_{2}\right)=Z\left(t_{1}\right) \rightarrow Z\left(t_{2}\right), Z\left(t_{1} \rightarrow_{1} t_{2}\right)=$ $\left[Z\left(t_{1}\right)\right] \rightarrow Z\left(t_{2}\right)$.

\section{The Mapping $C_{t}^{t^{\prime}}$}

To cope with the subsumption rule we need a function $C_{t}^{t^{\prime}}$, parametrized by strictness types $t$ and $t^{\prime}$ such that $t \leq t^{\prime}$, with the following intended property: if $e^{\prime}$ has type $Z(t)$, then $C_{t}^{t^{\prime}}\left(e^{\prime}\right)$ is an expression of type $Z\left(t^{\prime}\right)$.

Example 5.1 Suppose $t=\ln t \rightarrow_{0} \operatorname{Int}$ and $t^{\prime}=\ln t \rightarrow_{1} \operatorname{lnt}$, and suppose $e^{\prime}$ has type Int $\rightarrow \operatorname{lnt} . C_{t}^{t^{\prime}}\left(e^{\prime}\right)$ then has to be something of type [Int] $\rightarrow \operatorname{lnt}-$ it is easily seen that $\lambda x \cdot e^{\prime} \mathcal{D}(x)$ (with $x$ fresh) will do the job.

$C_{t}^{t^{\prime}}$ is defined as follows (inductively in the "size" of $t$ and $t^{\prime}$ ):

1. If $t=t^{\prime}$, then $C_{t}^{t^{\prime}}(e)=e$.

2. If $t=t_{1} \rightarrow_{0} t_{2}$ and $t^{\prime}=t_{1}^{\prime} \rightarrow_{0} t_{2}^{\prime}$ (with $t_{1}^{\prime} \leq t_{1}, t_{2} \leq t_{2}^{\prime}$ ), then (where $x$ is a "fresh" variable)

$$
C_{t}^{t^{\prime}}(e)=\lambda x \cdot C_{t_{2}}^{t_{2}^{\prime}}\left(e C_{t_{1}^{\prime}}^{t_{1}}(x)\right)
$$

3. If $t=t_{1} \rightarrow_{1} t_{2}$ and $t^{\prime}=t_{1}^{\prime} \rightarrow_{1} t_{2}^{\prime}$ (with $t_{1}^{\prime} \leq t_{1}, t_{2} \leq t_{2}^{\prime}$ ), then (where $x$ is a "fresh" variable)

$$
C_{t}^{t^{\prime}}(e)=\lambda x . C_{t_{2}}^{t_{2}^{\prime}}\left(e \underline{\left.C_{t_{1}^{\prime}}^{t_{1}}(\mathcal{D}(x))\right)}\right.
$$

4. If $t=t_{1} \rightarrow_{0} t_{2}$ and $t^{\prime}=t_{1}^{\prime} \rightarrow_{1} t_{2}^{\prime}$ (with $t_{1}^{\prime} \leq t_{1}, t_{2} \leq t_{2}^{\prime}$ ), then (where $x$ is a "fresh" variable)

$$
C_{t}^{t^{\prime}}(e)=\lambda x \cdot C_{t_{2}}^{t_{2}^{\prime}}\left(e C_{t_{1}^{\prime}}^{t_{1}}(\mathcal{D}(x))\right)
$$




\section{A Modified Inference System}

It will be convenient to elaborate slightly on the inference system presented in Fig. 4. The resulting system is depicted in Fig. 8. The changes performed are:

- An extra entity $T$ is introduced, such that judgements take the form $\Gamma^{\prime}, T \vdash_{s a} e: t, W$. Here $T$ is a subset of the domain of $\Gamma$; the purpose of $T$ is to record which variables have been bound by non-strict $\lambda$ 's.

- The two rules for abstraction and for application have been split into one for strict functions and one for non-strict.

\section{The Translation Algorithm}

Given an expression $e$, and a proof of $\Gamma, T \vdash_{s a} e: t, W$ using the rules in Fig. 8. We now present an algorithm for transforming $e$ into an expression $e^{\prime}$, with the aim that the "CBV-semantics" of $e^{\prime}$ should equal the "CBN-semantics" of $e$.

The translation is defined inductively in the proof tree - several cases:

- Suppose $\Gamma, T \vdash_{s a} e: t^{\prime}, W^{\prime}$ because $\Gamma, T \vdash_{s a} e: t, W$ and $t \leq t^{\prime}, W \leq$ $W^{\prime}$. Suppose $e$ (by the latter proof tree) transforms into $e^{\prime}$. Then $e$ (by the former proof tree) transforms into $C_{t}^{t^{\prime}}\left(e^{\prime}\right)$.

- Suppose $e=c$. Then we let $e^{\prime}=c$.

- Suppose $e=x$. Two cases:

- If $x \in T$, we let $e^{\prime}=\mathcal{D}(x)$ (as $x$ will be bound to a thunkified argument).

- If $x \notin T$, we let $e^{\prime}=x$.

- Suppose $e=\lambda x \cdot e_{1}$, and suppose $e_{1}$ (using the relevant proof tree) translates into $e_{1}^{\prime}$. Then $e$ translates into $\lambda x . e_{1}^{\prime}$.

- Suppose $e=e_{1} e_{2}$, and suppose $e_{1}$ and $e_{2}$ (using the relevant proof trees) translate into $e_{1}^{\prime}$ resp. $e_{2}^{\prime}$ Two cases: 


$$
\begin{aligned}
& \frac{\Gamma, T \vdash_{s a} e: t, W}{\Gamma, T \vdash_{s a} e: t^{\prime}, W^{\prime}} \text { if } t^{\prime} \geq t, W^{\prime} \geq W \\
& \Gamma, T \vdash_{s a} c: C T_{s a}(c), \overrightarrow{1} \\
& \left(\Gamma_{1},(x: t), \Gamma_{2}\right), T \vdash_{s a} x: t,(\overrightarrow{1}, 0, \overrightarrow{1}) \\
& \frac{\left(\left(x: t_{1}\right), \Gamma\right), T \vdash_{s a} e: t,(0, W)}{\Gamma, T \vdash_{s a} \lambda x \cdot e: t_{1} \rightarrow{ }_{0} t, W} \\
& \frac{\left(\left(x: t_{1}\right), \Gamma\right), T \cup\{x\} \vdash_{s a} e: t,(1, W)}{\Gamma, T \vdash_{s a} \lambda x \cdot e: t_{1} \rightarrow{ }_{1} t, W} \\
& \frac{\Gamma, T \vdash_{s a} e_{1}: t_{2} \rightarrow{ }_{0} t_{1}, W_{1} \Gamma, T \vdash_{s a} e_{2}: t_{2}, W_{2}}{\Gamma, T \vdash_{s a} e_{1} e_{2}: t_{1}, W} \text { if } W(x)=W_{1}(x) \sqcap W_{2}(x) \text { for all } x \\
& \frac{\Gamma, T \vdash_{s a} e_{1}: t_{2} \rightarrow{ }_{1} t_{1}, W_{1} \Gamma, T \vdash_{s a} e_{2}: t_{2}, W_{2}}{\Gamma, T \vdash_{s a} e_{1} e_{2}: t_{1}, W} \text { if } W(x)=W_{1}(x) \text { for all } x \\
& \frac{\Gamma, T \vdash_{s a} e_{1}: \text { Bool, } W_{1} \Gamma, T \vdash_{s a} e_{2}: t, W_{2} \Gamma, T \vdash_{s a} e_{3}: t, W_{3}}{\Gamma, T \vdash_{s a}\left(\text { if } e_{1} e_{2} e_{3}\right): t, W} \\
& \text { if } W(x)=W_{1}(x) \sqcap\left(W_{2}(x) \sqcup W_{3}(x)\right) \text { for all } x \\
& \frac{((f: t), \Gamma), T \vdash_{s a} e: t,(b, W)}{\Gamma, T \vdash_{s a}(\operatorname{rec} f e): t, W}
\end{aligned}
$$

Figure 8: An inference system for strictness types. 
- If $e_{1}$ is of type $t_{2} \rightarrow_{0} t_{1}, e$ translates into $e_{1}^{\prime} e_{2}^{\prime}$.

- If $e_{1}$ is of type $t_{2} \rightarrow_{1} t_{1}$, e translates into $e_{1}^{\prime} \underline{e_{2}^{\prime}}$.

- Suppose $e=$ if $e_{1} e_{2} e_{3}$, and suppose $e_{1}, e_{2}$ and $e_{3}$ (using the relevant proof trees) translate into $e_{1}^{\prime}, e_{2}^{\prime}$ resp. $e_{3}^{\prime}$. Then $e$ translates into if $e_{1}^{\prime} e_{2}^{\prime} e_{3}^{\prime}$

- Suppose $e=\operatorname{rec} f e_{1}$, and suppose $e_{1}$ (using the relevant proof tree) translates into $e_{1}^{\prime}$. Then $e$ translates into rec $f e_{1}^{\prime}$.

We see that if all arrows are annotated 1 (and correspondingly all $\lambda$-bound variables belong to $T$ ), we get the same code as produced by the "naive approach" (cf. Sect. 1.3). But if we are able to annotate some arrows 0 , better code (i.e. fewer thunkifications/dethunkifications) will be obtained.

Example 5.2 Consider the expression twice $=\lambda f . \lambda x . f(f x)$. $\boldsymbol{t}$ wice has strictness type

$$
\begin{gathered}
\left(\operatorname{Int} \rightarrow_{1} \operatorname{Int}\right) \rightarrow_{0}\left(\operatorname{Int} \rightarrow_{1} \operatorname{Int}\right) \text { because } \\
\left(\left(f: \operatorname{lnt} \rightarrow_{1} \operatorname{lnt}\right)\right), \emptyset \vdash_{s a} \lambda x . f(f x):\left(\operatorname{Int} \rightarrow_{1} \operatorname{Int}\right),\{f\} \text { and } \\
\left((x: \operatorname{lnt}),\left(f:=\operatorname{lnt} \rightarrow_{1} \operatorname{Int}\right)\right),\{x\} \vdash_{s a} f(f x): \operatorname{Int},\{f\} \text { etc. }
\end{gathered}
$$

Accordingly, twice translates into the term

$$
\lambda f . \lambda x . f \underline{\underline{f \mathcal{D}(x)}}
$$

of type $([\ln t] \rightarrow \operatorname{lnt}) \rightarrow([\operatorname{lnt}] \rightarrow \ln t)$. We see that there is room for some (peephole) optimization here, as $\mathcal{D}(x)$ could be replaced by $x$.

Notice that $t$ wice also has strictness type $\left(\operatorname{Int} \rightarrow_{0} \operatorname{Int}\right) \rightarrow_{0}\left(\operatorname{Int} \rightarrow_{0} \operatorname{Int}\right)$. Using the corresponding proof tree, twice just translates into itself.

\section{Proving the Translation Correct}

Before proving the translation correct, one of course has to define what correctness means - this will be the topic of the next section. 


\subsection{Correctness Predicates}

Initially we shall consider closed expressions only. We will define a predicate $\sim_{t}$, indexed over strictness types, such that $q \sim_{t} q^{\prime}$ is defined whenever $q$ is a closed expression of type $\mathrm{E}(t)$, and $q^{\prime}$ is a closed expression of type $Z(t)$. $\sim_{t}$ is defined inductively on $t$ :

- $q \sim_{\text {Base }} q^{\prime}$ holds iff for all constants $c$ we have $q \Rightarrow_{N}^{*} c$ iff $q^{\prime} \Rightarrow_{V}^{*} c$ (in particular, $q$ loops by CBN iff $q^{\prime}$ loops by CBV).

- $q \sim_{t_{1} \rightarrow 0 t_{2}} q_{1}^{\prime}$ holds iff for all $q_{2} q_{2}^{\prime}$ such that $q_{2} \sim_{t_{1}} q_{2}^{\prime}$ we have $q_{1} q_{2} \sim_{t_{1}}$ $q_{1}^{\prime} q_{2}^{\prime}$.

- $q \sim_{t_{1} \rightarrow t_{2}} q_{1}^{\prime}$ holds iff for all $q_{2} q_{2}^{\prime}$ such that $q_{2} \sim_{t_{1}} q_{2}^{\prime}$ we have $q_{1} q_{2} \sim_{t_{1}}$ $q_{1}^{\prime} q_{2}^{\prime}$.

This very much resembles a logical relation, but notice the difference between $\sim_{t_{1} \rightarrow 0} t_{2}$ and $\sim_{t_{1} \rightarrow{ }_{1} t_{2}}$.

Now we are ready to consider arbitrary (non-closed) expressions. The main correctness predicate takes the form $e \operatorname{COR}(t, W, \Gamma, T) e^{\prime}$, where $e$ and $e^{\prime}$ are expressions and where $\Gamma, T \vdash_{s a} e: t, W$. We shall need an auxiliary function $Z_{T}$, mapping from $\mathcal{T}_{\text {sa }}$-environments into $\mathcal{T}$-environments: $Z_{T}(\Gamma)(x)=Z(\Gamma(x))$ for $x \notin T$; and $Z_{T}(\Gamma)(x)=[Z(\Gamma(x))]$ for $x \in T$.

Definition 6.1 e $\operatorname{COR}(t, W, \Gamma, T) e^{\prime}$ holds iff (with $\left\{x_{1} \ldots x_{n}\right\}$ being the domain of $\Gamma$ )

1. $Z_{T}(\Gamma) t \vdash e^{\prime}: Z(t)$.

2. $\mathrm{FV}(e)=\mathrm{FV}\left(e^{\prime}\right)$.

3. Let in the following closed terms $q_{i}, q_{i}^{\prime}(i \in\{1 \ldots n\})$ be such that $q_{i} \sim_{\Gamma\left(x_{i}\right)} q_{i}^{\prime}$. Then it must hold that

$$
e\left[\left\{q_{1} \ldots q_{n}\right\} /\left\{x_{1} \ldots x_{n}\right\}\right] \sim_{t} e^{\prime}\left[\left\{Q_{1}^{\prime} \ldots Q_{n}^{\prime}\right\} /\left\{x_{1} \ldots x_{n}\right\}\right]
$$

where $Q_{i}^{\prime}$ is defined as follows: if $x_{i} \in T$ then $Q_{i}^{\prime}=q_{i}^{\prime}$ else $Q_{i}^{\prime}=q_{i}^{\prime}$.

Suppose now that for some $i$ we have that $W\left(x_{i}\right)=0$ and $q_{i} \sim_{\Gamma(i)} \Omega$. Then 


$$
e\left[\left\{q_{1} \ldots q_{n}\right\} /\left\{x_{1} \ldots x_{n}\right\}\right] \sim_{t} \Omega
$$

The first part of 3 resembles the standard way of extending relations from closed terms to open terms; the second part of 3 expresses that the variables which by $W$ are mapped into 0 in fact are "needed".

\subsection{Introducing Bounded Recursion}

For technical reasons we shall introduce bounded recursion. That is, we add constructs of form $\operatorname{rec}_{n} f e$ (with $n \geq 0$ ) to the language (the old construct rec $f e$ is now termed unbounded recursion). This device is motivated by the SOS-rule rec $f e \Rightarrow_{N} e[(\operatorname{rec} f e) / f]$, where we want to (inductively) use properties of the latter rec to prove properties of the former rec.

Wrt. typing properties, rec $_{n}$ behaves exactly as rec. Wrt. translation, rec $_{n} f e$ translates into rec $_{n} f e^{\prime}$ (where $e^{\prime}$ is the translation of $e$ ). Wrt. semantics, we have the SOS-rules

$$
\begin{array}{rll}
\operatorname{rec}_{0} f e & \Rightarrow_{N} & \operatorname{rec}_{0} f e \\
\operatorname{rec}_{n+1} f e & \Rightarrow_{N} & \left.e\left[\operatorname{rec}_{0} f e\right] / f\right]
\end{array}
$$

and similarly for $\Rightarrow_{V}^{*}$.

\subsection{Correctness theorems}

The main effort will be to prove the following theorem:

Theorem 6.2 Suppose $\Gamma, T \vdash_{s a} e: t, W$, suppose $e$ contains no unbounded recursion (i.e. only rec ${ }_{n}$ 's and no rec's) and suppose $e$ (by means of the corresponding proof tree) translates into $e^{\prime}$. Then $e \operatorname{COR}(t, W, \Gamma, T) e^{\prime}$.

As the user certainly will like to use unbounded recursion, Theorem 6.2 may seem to be of limited use. However, it actually enables us to prove what we are really looking for: 
Theorem 6.3 Suppose $q$ is a closed expression (which may contain unbounded recursion) such that ()$, \emptyset \vdash_{s a} q$ : Base, (). Let $q^{\prime}$ be the translation of $q$, using the algorithm in Section 5. Now for all constants (of base type) $c, q \Rightarrow_{N}^{*} c$ iff $c, q^{\prime} \Rightarrow_{V}^{*} c$.

Proof: First some notation: given $n$, let $q_{n}$ be the result of substituting rec $_{n}$ for all occurrences of rec. It is easy to see that $q_{n}$ translates into $q_{n}^{\prime}$.

First (the "only if" part) suppose $q \Rightarrow_{N}^{*} c$. It is easy to see that there exists $n$ such that $q_{n} \Rightarrow_{N}^{*} c$. Since $q_{n}$ and $q_{n}^{\prime}$ does not contain unbounded recursion, Theorem 6.2 tells us that $q_{n} \operatorname{COR}($ Base, ()$,(), \emptyset) q_{n}^{\prime}$. This implies that $q_{n} \sim_{\text {Base }} q_{n}^{\prime}$ so $q_{n}^{\prime} \Rightarrow_{V}^{*} c$. But then it is immediate that $q^{\prime} \Rightarrow_{V}^{*} c$.

The "if" part is analogous.

We now embark on proving Theorem 6.2. The proof proceeds roughly speaking as follows:

1. In Sect. 6.4, a number of properties of $\sim_{t}$ are proved (by induction on $t$ ). For instance, we have that if $q \Rightarrow_{N} q_{1}$ and $q \sim_{t} q^{\prime}$ then also $q_{1} \sim_{t} q^{\prime}$.

2. In Sect. 6.5, some properties of $C_{t}^{t^{\prime}}$ are formulated and proved - for instance that if $q \sim_{t} q^{\prime}$ then $q \sim_{t^{\prime}} C_{t}^{t^{\prime}}\left(q^{\prime}\right)$.

3. Finally, in Sect. 6.6 we are able to prove Theorem 6.2 by induction in the proof tree.

\subsection{Some Lemmas concerning $\sim_{t}$}

Lemma 6.4 Let $q \sim_{t} q^{\prime}$, let $q_{1}$ be of type $\mathrm{E}(t)$ and let $q_{1}^{\prime}$ be of type $Z(t)$.

1. If $q^{\prime}$ and $q_{1}^{\prime}$ both loop by $\Rightarrow_{V}, q \sim_{t} q_{1}^{\prime}$.

2. If $q$ and $q_{1}$ both loop by $\Rightarrow_{N}, q_{1} \sim_{t} q^{\prime}$.

Proof: We only consider 1 (2 is analogous). We use induction in $t$; first consider when $t$ is a base type. Then we can infer that $q$ loops by $\Rightarrow_{N}$, implying that $q \sim_{t} q_{1}^{\prime}$. 
Next assume $t=t_{2} \rightarrow_{0} t_{1}$ (The case $t=t_{2} \rightarrow_{1} t_{1}$ is analogous). Then we must show that if $q_{2} \sim_{t_{2}} q_{2}^{\prime}$ then $q q_{2} \sim_{t_{1}} q_{1}^{\prime} q_{2}^{\prime}$ But we know that $q q_{2} \sim_{t_{1}} q^{\prime} q_{2}^{\prime}$. As $q^{\prime} q_{2}^{\prime}$ and $q_{1}^{\prime} q_{2}^{\prime}$ both loop by $\Rightarrow_{V}$, the induction hypothesis applied to $t_{1}$ gives the claim.

Lemma 6.5 Let $q$ be of type $\mathrm{E}(t)$ and let $q^{\prime}$ be of type $Z(t)$. If $q$ loops by $\Rightarrow_{N}$ and $q^{\prime}$ loops by $\Rightarrow_{V}$ we have $q \sim_{t} q^{\prime}$.

Proof: Induction in $t$. If $t$ is a base type, it is obvious. Now assume $t=t_{2} \rightarrow_{0} t_{1}$ (the case $t=t_{2} \rightarrow_{1} t_{1}$ is analogous). Then we have to show that $q q_{2} \sim_{t_{1}} q^{\prime} q_{2}^{\prime}$, whenever $q_{2} \sim_{t_{2}} q_{2}^{\prime}$. But $q q_{2}$ loops by $\Rightarrow_{N}$ and $q^{\prime} q_{2}^{\prime}$ loops by $\Rightarrow_{V}$, so this follows from the induction hypothesis applied to $t_{1}$.

Lemma 6.6 Suppose $q \Rightarrow_{N} q_{1}$. Then $q \sim_{t} q^{\prime}$ iff $q_{1} \sim_{t} q^{\prime}$. Suppose $q^{\prime} \Rightarrow_{V} q_{1}^{\prime}$. Then $q \sim_{t} q^{\prime}$ iff $q_{1} \sim_{t} q_{1}^{\prime}$.

Proof: We only show the first part - by induction in $t$ : For base types, it is obvious as $\Rightarrow_{N}$ is deterministic. So assume that $t=t_{2} \rightarrow_{0} t_{1}$ (the case $t=t_{2} \rightarrow_{1} t_{1}$ is analogous). It is enough if we can show that if $q_{2} \sim_{t_{2}} q_{2}^{\prime}$ then $q q_{2} \sim_{t_{1}} q^{\prime} q_{2}^{\prime}$ iff $q_{1} q_{2} \sim_{t_{1}} q^{\prime} q_{2}^{\prime}$. But as $q q_{2} \Rightarrow_{N} q_{1} q_{2}$, the induction hypothesis yields the desired result.

Lemma 6.7 For all constants $c, c \sim C T_{s a(c)} c$.

Proof: Induction in $t=C T_{s a}(c)$. If it is a base type, it is trivial. Otherwise, our requirements to constants tell us that $t=$ Base $\rightarrow_{0} t_{2}$. So assume that $q \sim_{\text {Base }} q^{\prime}$ then we must show that $c q \sim_{t_{2}} c q^{\prime}$. Two possibilities:

- There exists a constant $w$ such that $q \Rightarrow_{N}^{*} w$. Then also $q^{\prime} \Rightarrow_{V}^{*} w$. By Lemma 6.6 it will be enough to show that $c w \sim_{t_{2}} c w$, and again by applying Lemma 6.6 we see that it is enough to show that Applycon $(c, w) \sim_{t_{2}} \operatorname{Applycon}(c, w)$. If $\operatorname{Applycon}(c, w)$ yields a constant this follows from the induction hypothesis applied to $t_{2}-$ if $\operatorname{Applycon}(c, w)=$ $c w$ then use Lemma 6.5.

- $q$ loops by $\Rightarrow_{N}$. Then also $q^{\prime}$ loops by $\Rightarrow_{V}$. This implies that $c q$ loops by $\Rightarrow_{N}$ and that $c q^{\prime}$ loops by $\Rightarrow_{V}$, so we can apply Lemma 65 . 
Lemma 6.8 Suppose that it holds that $q \sim_{t} q^{\prime}$ implies that $e[q / f] \sim_{t} e^{\prime}\left[q^{\prime} / f\right]$ (the latter being closed terms). Then for all $n, \operatorname{rec}_{n} f e \sim_{t} \operatorname{rec}_{n} f e^{\prime}$.

Proof: Induction in $n$. If $n=0$, both sides loop so we can apply Lemma 6.5. In the induction case, it by Lemma 6.6 is enough to show that

$$
e\left[\operatorname{rec}_{n-1} f e / f\right] \sim_{t} e^{\prime}\left[\operatorname{rec}_{n-1} f e^{\prime} / f\right]
$$

But this follows from the assumptions and the induction hypothesis.

Lemma 6.9 Suppose it for all $q$ such that there exists $q^{\prime}$ with $q \sim_{t} q^{\prime}$ holds that $e[q / f] \sim_{t} \Omega$ (where $\operatorname{FV}(e) \subseteq\{x\}$.) Then for all $n$, $\operatorname{rec}_{n} f e \sim_{t} \Omega$.

Proof: Induction in $n$. If $n=0$, $\operatorname{rec}_{n} f e$ loops and the claim follows from Lemma 6.5. If $n>0$, it by Lemma 6.6 is enough to show that $e\left[\operatorname{rec}_{n-1} f e / f\right] \sim_{t} \Omega$. But this follows from the assumption of the lemma and the induction hypothesis.

\subsection{Correctness of $C_{t}^{t^{\prime}}$}

Lemma 6.10 For all strictness types $t$ and $t^{\prime}$ with $t \leq t^{\prime}, C_{t}^{t^{\prime}}$ satisfies the following properties:

1. $\mathrm{FV}(e)=\mathrm{FV}\left(C_{t}^{t^{\prime}}(e)\right)$

2. With symbols having appropriate types,

$$
C_{t}^{t^{\prime}}(e)\left[\left\{q_{1} \ldots q_{n}\right\} /\left\{x_{1} \ldots x_{n}\right\}\right]=C_{t}^{t^{\prime}}\left(e\left[\left\{q_{1} \ldots q_{n}\right\} /\left\{x_{1} \ldots x_{n}\right\}\right]\right)
$$

3. If $\Gamma \vdash e: Z(t)$, then $\Gamma \vdash C_{t}^{t^{\prime}}(e): Z\left(t^{\prime}\right)$.

4. Suppose $q \sim_{t} \Omega$. Then also $q \sim_{t^{\prime}} \Omega$. (here $C_{t}^{t^{\prime}}$ is not involved, but this is needed for technical purposes).

5. Suppose $q \sim_{t} q^{\prime}$. Then also $q \sim_{t^{\prime}} C_{t}^{t^{\prime}}\left(q^{\prime}\right)$. 
Proof: Induction in $t$ (in the "number of arrows"). That property 1 and 2 hold is easy to see. Now consider property 3. Three cases:

- $t=t_{1} \rightarrow_{0} t_{2}, t^{\prime}=t_{1}^{\prime} \rightarrow_{0} t_{2}^{\prime}$. We know that $\Gamma \vdash e: Z\left(t_{1}\right) \rightarrow Z\left(t_{2}\right)$ and must show that

$$
\left.\Gamma \vdash \lambda x . C_{t_{2}}^{t_{2}^{\prime}}\left(e C_{t_{1}^{\prime}}^{t_{1}}\right)\right): Z\left(t_{1}^{\prime}\right) \rightarrow Z\left(t_{2}^{\prime}\right)
$$

But this will easily follow from the induction hypothesis.

- $t=t_{1} \rightarrow_{1} t_{2}, t^{\prime}=t_{1}^{\prime} \rightarrow_{1} t_{2}^{\prime}$. We know that $\Gamma \vdash e:\left[Z\left(t_{1}\right)\right] \rightarrow Z\left(t_{2}\right)$, and must show that

$$
\Gamma \vdash \lambda x . C_{t_{2}}^{t_{2}^{\prime}}\left(e C_{t_{1}^{\prime}}^{t_{1}}(\mathcal{D}(x))\right):\left[Z\left(t_{1}^{\prime}\right)\right] \rightarrow Z\left(t_{2}^{\prime}\right)
$$

Also this follows easily from the induction hypothesis.

- $t=t_{1} \rightarrow_{0} t_{2}, t^{\prime}=t_{1}^{\prime} \rightarrow_{1} t_{2}^{\prime}$. We know that $\Gamma \vdash e: Z\left(t_{1}\right) \rightarrow Z\left(t_{2}\right)$ and must show that

$$
\Gamma \vdash \lambda x . C_{t_{2}}^{t_{2}^{\prime}}\left(e C_{t_{1}^{\prime}}^{t_{1}}(\mathcal{D}(x))\right):\left[Z\left(t_{1}^{\prime}\right)\right] \rightarrow Z\left(t_{2}^{\prime}\right)
$$

which follows easily from the induction hypothesis.

Next for property 4 , where we can assume that $t=t_{1} \rightarrow_{0} t_{2}$ and $t^{\prime}=t_{1}^{\prime} \rightarrow_{1} t_{2}^{\prime}$ with $t_{1}^{\prime} \leq t_{1}, t_{2} \leq t_{2}^{\prime}$ (the other cases are similar). We know that $q \sim_{t} \Omega$. In order to verify that $q \sim_{t^{\prime}} \Omega$, assume that $q_{1} \sim_{t_{1}^{\prime}} q_{1}^{\prime}$ - then we must show that $q q_{1} \sim_{t_{2}^{\prime}} \Omega q_{1}^{\prime}$. Inductively we can assume that property 5 holds for $t_{1}^{\prime}$, so $q_{1} \sim_{t_{1}} C_{t_{1}^{\prime}}^{t_{1}}$. Then $q q_{1} \sim_{t_{2}} \Omega C_{t_{1}^{\prime}}^{t_{1}}\left(q_{1}^{\prime}\right)$ i.e. (by Lemma 6.4) that $q q_{1} \sim_{t_{2}} \Omega$. Inductively we can assume that property 4 holds for $t_{2}$, so $q q_{1} \sim_{t_{2}^{\prime}} \Omega$-which by Lemma 6.4 is the desired result.

Finally, we have to check that property 5 holds. 3 cases:

- $t=t_{1} \rightarrow_{0} t_{2}, t^{\prime}=t_{1}^{\prime} \rightarrow_{1} t_{2}^{\prime}$. Since $q \sim_{t} q^{\prime}$ we know that

$$
q_{1} \sim_{t_{1}} q_{1}^{\prime} \text { implies that } q q_{1} \sim_{t_{2}} q^{\prime} q_{1}^{\prime}
$$

Assuming that $q_{1} \sim_{t_{1}^{\prime}} q_{1}^{\prime}$, our task is to show that

$$
q q_{1} \sim_{t_{2}^{\prime}}\left(\lambda x C_{t_{2}}^{t_{2}^{\prime}}\left(q^{\prime} C_{t_{1}^{\prime}}^{t_{1}}(x)\right)\right) q_{1}^{\prime}
$$

Now two possibilities: 
- $q_{1}^{\prime}$ loops by $\Rightarrow_{V}$. By Lemma 6.4, $q_{1} \sim_{t_{1}^{\prime}} \Omega$. Inductively we can assume that property 4 holds for $t_{1}^{\prime}$, so $q_{1} \sim_{t_{1}} \Omega$. By (1), this implies that $q q_{1} \sim_{t_{2}} q^{\prime} \Omega$. By Lemma 6.4, this (as $q^{\prime} \Omega$ loops by $\Rightarrow_{V}$ ) amounts to $q q_{1} \sim_{t_{2}} \Omega$. But by Lemma 6.4 , this is just (2).

- There exists a $w_{1}^{\prime}$ in WHNF such that $q_{1}^{\prime} \Rightarrow_{V}^{*} w_{1}^{\prime}$. By repeated application of Lemma 6.6, it in order to show (2) is enough to show that $q q_{1} \sim_{t_{2}^{\prime}} C_{t_{2}}^{t_{2}}\left(q^{\prime} C_{t_{1}^{\prime}}^{t_{1}^{\prime}}(x)\right)\left[w_{1}^{\prime} / x\right]$ which (as we can assume that property 2 holds) amounts to showing that $q q_{1} \sim_{t_{2}^{\prime}}$ $C_{t_{2}}^{t_{2}}\left(q^{\prime} C_{t_{1}^{\prime}}^{t_{1}^{\prime}}\left(w_{1}^{\prime}\right)\right)$. By the induction hypothesis applied to $t_{2}$, this can be done by showing that $q q_{1} \sim_{t_{2}^{\prime}} q^{\prime} C_{t_{1}^{\prime}}^{t_{1}^{\prime}}\left(w_{1}^{\prime}\right)$. As (1) holds, it is enough to show that $q_{1} \sim_{t_{1}} C_{t_{1}^{\prime}}^{t_{1}}\left(w_{1}^{\prime}\right)$ which by the induction hypothesis applied to $t_{1}^{\prime}$ can be done by showing $q_{1} \sim_{t_{1}^{\prime}} w_{1}^{\prime}$ But this follows (from $q_{1} \sim_{t_{1}^{\prime}} q_{1}^{\prime}$ ) by repeated application of Lemma 6.6 .

- $t=t_{1} \rightarrow_{1} t_{2}, t^{\prime}=t_{1}^{\prime} \rightarrow_{1} t_{2}^{\prime}$. Since $q \sim_{t} q^{\prime}$ we know that

$$
q_{1} \sim_{t_{1}} q_{1}^{\prime} \text { implies that } q q_{1} \sim_{t_{2}} q^{\prime} \underline{q_{1}^{\prime}}
$$

Assuming that $q_{1} \sim_{t_{1}^{\prime}} q_{1}^{\prime}$, our task is to show that

$$
q q_{1} \sim_{t_{2}^{\prime}}\left(\lambda x \cdot C_{t_{2}}^{t_{2}^{\prime}}\left(q^{\prime} C_{t_{1}^{\prime}}^{t_{1}}(\mathcal{D}(x))\right)\right) \underline{q_{1}^{\prime}} .
$$

Lemma 6.6 says (as $q_{1}^{\prime}$ is in WHNF) that is sufficient to show that $\left.q q_{1} \sim_{t_{2}^{\prime}} C_{t_{2}}^{t_{2}^{\prime}}\left(q^{\prime} C_{t_{1}^{\prime}}^{t_{1}}(\mathcal{D}(x))\right)\right)\left[\underline{q_{1}^{\prime}} / x\right]$ which (as we can assume that property 2 holds) amounts to showing that $\left.q q_{1} \sim_{t_{2}^{\prime}} C_{t_{2}}^{t_{2}^{\prime}}\left(q^{\prime} C_{t_{1}^{\prime}}^{t_{1}}\left(\mathcal{D}\left(\underline{q_{1}^{\prime}}\right)\right)\right)\right)$. By the induction hypothesis applied to $t_{2}$, this can be done by showing that $q q_{1} \sim_{t_{2}} q^{\prime} C_{t_{1}^{\prime}}^{t_{1}}\left(\mathcal{D}\left(\underline{q_{1}^{\prime}}\right)\right)$. As (3) holds, it is enough to show that $q_{1} \sim_{t_{1}} C_{t_{1}^{\prime}}^{t_{1}}\left(\mathcal{D}\left(\underline{q_{1}^{\prime}}\right)\right)$ which by the induction hypothesis applied to $t_{1}^{\prime}$ can be done by showing $q_{1} \sim_{t_{1}^{\prime}} \mathcal{D}\left(\underline{q_{1}^{\prime}}\right)$. But this follows (from $q_{1} \sim_{t_{1}^{\prime}} q_{1}^{\prime}$ ) by Lemma 6.6 and Fact 2.2.

- $t=t_{1} \rightarrow_{0} t_{2}, t^{\prime}=t_{1}^{\prime} \rightarrow_{1} t_{2}^{\prime}$. Since $q \sim_{t} q^{\prime}$ we know that

$$
q_{1} \sim_{t_{1}} q_{1}^{\prime} \text { implies that } q q_{1} \sim_{t_{2}} q^{\prime} q_{1}^{\prime}
$$

Assuming that $q_{1} \sim_{t_{1}^{\prime}} q_{1}^{\prime}$ our task is to show that 


$$
q q_{1} \sim_{t_{2}^{\prime}}\left(\lambda . x C_{t_{2}}^{t_{2}^{\prime}}\left(q^{\prime} C_{t_{1}^{\prime}}^{t_{1}}(\mathcal{D}(x))\right)\right) \underline{q_{1}^{\prime}} .
$$

Lemma 6.6 says (as $q_{1}^{\prime}$ is in WHNF) that is sufficient to show that $q q_{1} \sim_{t_{2}^{\prime}} C_{t_{2}}^{t_{2}^{\prime}}\left(q^{\prime} C_{t_{1}^{\prime}}^{t_{1}}(\mathcal{D}(x))\right)\left[\underline{q_{1}^{\prime}} / x\right]$ which (as we can assume that property 2 holds) amounts to showing that $q q_{1} \sim_{t_{2}^{\prime}} C_{t_{2}}^{t_{2}^{\prime}}\left(q^{\prime} C_{t_{1}^{\prime}}^{t_{1}}\left(\mathcal{D}\left(q_{1}^{\prime}\right)\right)\right)$. By the induction hypothesis applied to $t_{2}$, this can be done by showing that $q q_{1} \sim_{t_{2}^{\prime}} q^{\prime} C_{t_{1}^{\prime}}^{t_{1}}\left(\mathcal{D}\left(\underline{q_{1}^{\prime}}\right)\right)$. As (4) holds, it is enough to show that $q_{1} \sim_{t_{1}}$ $C_{t_{1}^{\prime}}^{t_{1}}\left(\mathcal{D}\left(\underline{q_{1}^{\prime}}\right)\right)$ which by the induction hypothesis applied to $t_{1}^{\prime}$ can be done by showing $q_{1} \sim_{t_{1}^{\prime}} \mathcal{D}\left(q_{1}^{\prime}\right)$. But this follows (from $q_{1} \sim_{t_{1}^{\prime}} q_{1}^{\prime}$ ) by Lemma 6.6 and Fact 2.2.

\subsection{Proof of Theorem 6.2}

We will proceed by induction in the proof tree for

$$
\Gamma, T \vdash_{s a} e: t, W
$$

(using the inference system in Fig. 8). The non-trivial parts will be to show that

- $Z_{T}(\Gamma) \vdash e^{\prime}: Z(t)$.

- If $q_{i} \sim_{\Gamma\left(x_{i}\right)} q_{i}^{\prime}$ then

$$
e\left[\left\{q_{1} \ldots q_{n}\right\} /\left\{x_{1} \ldots x_{n}\right\}\right] \sim_{t} e^{\prime}\left[\left\{Q_{1}^{\prime} \ldots Q_{n}^{\prime}\right\} /\left\{x_{1} \ldots x_{n}\right\}\right]
$$

where $Q_{i}^{\prime}=q_{i}^{\prime}$ if $x_{i} \in T ; Q_{i}^{\prime}=q_{i}^{\prime}$ otherwise. Moreover, if $W\left(x_{i}\right)=0$ and $q_{i} \sim_{\Gamma\left(x_{i}\right)} \bar{\Omega}$ then

$$
e\left[\left\{q_{1} \ldots q_{n}\right\} /\left\{x_{1} \ldots x_{n}\right\}\right] \sim_{t} \Omega
$$

We split into several cases: 
- Suppose $\Gamma, T \vdash_{s a} e: t^{\prime}, W^{\prime}$ because $\Gamma, T \vdash_{s a} e: t, W, t \leq t^{\prime}, W^{\prime} \geq W$. We must show that

$$
Z_{T}(\Gamma) \vdash C_{t}^{t^{\prime}}\left(e^{\prime}\right): Z\left(t^{\prime}\right)
$$

but by Lemma 6.10 this follows from the induction hypothesis.

Next we must show that if $q_{i} \sim_{\Gamma\left(x_{i}\right)} q_{i}^{\prime}$, then

$$
e\left[\left\{q_{1} \ldots q_{n}\right\} /\left\{x_{1} \ldots x_{n}\right\}\right] \sim_{t^{\prime}} C_{t}^{t^{\prime}}\left(e^{\prime}\right)\left[\left\{Q_{1}^{\prime} \ldots Q_{n}^{\prime}\right\} /\left\{x_{1} \ldots x_{n}\right\}\right]
$$

But again this follows from the induction hypothesis and Lemma 6.10. Finally we must show that if $W^{\prime}\left(x_{i}\right)=0$ and $q_{i} \sim_{\Gamma\left(x_{i}\right)} \Omega$ then $e\left[\left\{q_{1} \ldots q_{n}\right\} /\left\{x_{1} \ldots x_{n}\right\}\right] \sim_{t^{\prime}} \Omega$. But this follows from the induction hypothesis and Lemma 6.10 , as $W^{\prime} \geq W$ so also $W\left(x_{i}\right)=0$.

- Suppose $\Gamma, T \vdash_{s a} c$ : $t, \overrightarrow{1}$ with $t=C T_{s a}(c)$. $c$ translates into $c$. We must show that $Z_{t}(\Gamma) \vdash c: Z(t)$ but this is obvious as $t=C t(c)[\overrightarrow{0},()]$ so $Z(t)=C t(c)$.

Next we must show that $c \sim_{t} c$ but this is the content of Lemma 6.7

- Suppose $\Gamma, T \vdash_{s a} x$ : $t, W$ with $\Gamma=\left(\Gamma_{1},(x: t), \Gamma_{2}\right), W=(\overrightarrow{1}, 0, \overrightarrow{1})$ Let $i 0$ be such that $x=x_{i 0}$. Two cases:

$-x \notin T$ : then $x$ translates into $x$. We must show that $Z_{T}(\Gamma) \vdash x$ : $Z(t)$ but this is obvious as $x \notin T$.

Next we must show that if $q_{i} \sim_{\Gamma\left(x_{i}\right)} q_{i}^{\prime}$ then

$$
x\left[\left\{q_{1} \ldots q_{n}\right\} /\left\{x_{1} \ldots x_{n}\right\}\right] \sim_{t} x\left[\left\{Q_{1}^{\prime} \ldots Q_{n}^{\prime}\right\} /\left\{x_{1} \ldots x_{n}\right\}\right]
$$

which amounts to showing that $q_{i 0} \sim_{t} q_{i 0}^{\prime}$ which follows trivially from the assumptions.

Finally, we must show that if $q_{i 0} \sim_{\Gamma(x)} \Omega$ then also $x\left[\left\{q_{1} \ldots q_{n}\right\} /\left\{x_{1} \ldots x_{n}\right\}\right] \sim_{t} \Omega-$ but this is trivial.

$-x \in T$ : then $x$ translates into $\mathcal{D}(x)$. We must show that $Z_{T}(\Gamma) \vdash$ $\mathcal{D}(x): Z(t)$ but this is obvious since $Z_{T}(\Gamma)(x)=\left[Z_{T}(\Gamma(x))\right]$.

Next we must show that if $q_{i} \sim_{\Gamma\left(x_{i}\right)} q_{i}^{\prime}$ then

$$
x\left[\left\{q_{1} \ldots q_{n}\right\} /\left\{x_{1} \ldots x_{n}\right\}\right] \sim_{t} \mathcal{D}(x)\left[\left\{Q_{1}^{\prime} \ldots Q_{n}^{\prime}\right\} /\left\{x_{1} \ldots x_{n}\right\}\right]
$$


which amounts to showing that $q_{i 0} \sim_{t} \mathcal{D}\left(\underline{q_{i 0}^{\prime}}\right)$ which follows from Lemma 6.6 and Fact 2.2.

Finally, we must show that if $q_{i} \sim_{\Gamma(x)} \Omega$ then also $x\left[\left\{q_{1} \ldots q_{n}\right\} /\left\{x_{1} \ldots x_{n}\right\}\right] \sim_{t} \Omega-$ but this is trivial.

- Suppose $\Gamma, T \vdash_{s a} \lambda x . e: t_{1} \rightarrow_{0} t, W$ because $\left(\left(x: t_{1}\right), \Gamma\right), T \vdash_{s a} e$ : $t,(0, W)$. Here $\lambda x$.e translates into $\lambda x . e^{\prime}$, where $e$ translates into $e^{\prime}$.

Our first task is to show that $Z_{T}(\Gamma) \vdash \lambda x . e^{\prime}: Z\left(t_{1} \rightarrow_{0} t\right)$. This can be done by showing that $\left(\left(x: Z\left(t_{1}\right)\right), Z_{T}(\Gamma)\right) \vdash e^{\prime}: Z(t)$ but as $\left(\left(x: Z\left(t_{1}\right), Z_{T}(\Gamma)\right)=Z_{T}\left(\left(x: t_{1}\right), \Gamma\right)\right.$ this follows from $e \operatorname{COR}\left(t,,_{-},,_{-}\right) e^{\prime}$.

Now suppose $q_{i} \sim_{\Gamma\left(x_{i}\right)} q_{i}^{\prime}$. We have to show that

$$
\lambda x .\left(e\left[\left\{q_{1} \ldots q_{n}\right\} /\left\{x_{1} \ldots x_{n}\right\}\right]\right) \sim_{t_{1} \rightarrow 0 t} \lambda x .\left(e^{\prime}\left[\left\{Q_{1}^{\prime} \ldots Q_{n}^{\prime}\right\} /\left\{x_{1} \ldots x_{n}\right\}\right]\right)
$$

Assuming that $q \sim_{t_{1}} q^{\prime}$, this amounts to showing

$$
\lambda x .\left(e\left[\left\{q_{1} \ldots q_{n}\right\} /\left\{x_{1} \ldots x_{n}\right\}\right]\right) q \sim_{t} \lambda x .\left(e^{\prime}\left[\left\{Q_{1}^{\prime} \ldots Q_{n}^{\prime}\right\} /\left\{x_{1} \ldots x_{n}\right\}\right]\right) q^{\prime}
$$

Now we split into two cases:

- Suppose $q^{\prime}$ loops by CBV. Then $q \sim_{t} \Omega$. Since $e \operatorname{COR}\left(t,(0, W),_{-},,_{-}\right)$ $e^{\prime}$, we can conclude that

$$
\left.e\left[\left\{q, q_{1} \ldots q_{n}\right\} /\left\{x, x_{1} \ldots x_{n}\right\}\right]\right) \sim_{t} \Omega
$$

By Lemma 6.6, we can conclude that

$$
\lambda x .\left(e\left[\left\{q_{1} \ldots q_{n}\right\} /\left\{x_{1} \ldots x_{n}\right\}\right]\right) q \sim_{t} \Omega
$$

and by Lemma 6.4 this yields the desired.

- Suppose $q^{\prime} \Rightarrow_{V}^{*} w^{\prime}$, with $w^{\prime}$ in WHNF. Then our task (by Lemma 6.6) amounts to showing that

$$
\left.e\left[\left\{q, q_{1} \ldots q_{n}\right\} /\left\{x, x_{1} \ldots x_{n}\right\}\right]\right) \sim_{t} e^{\prime}\left[\left\{w^{\prime}, Q_{1}^{\prime} \ldots Q_{n}^{\prime}\right\} /\left\{x, x_{1} \ldots x_{n}\right\}\right]
$$

But since (by Lemma 6.6) $q \sim_{t_{1}} w^{\prime}$, this follows from $e \operatorname{COR}\left(t,,_{-},-T\right)$ $e^{\prime}$ (with $\left.x \notin T\right)$.

Finally, we have to show that if $W\left(x_{i}\right)=0, q_{i} \sim_{\Gamma\left(x_{i}\right)} \Omega$ then

$$
\lambda x . e\left[\left\{q_{1} \ldots q_{n}\right\} /\left\{x_{1} \ldots x_{n}\right\}\right] \sim_{t_{1} \rightarrow 0} \Omega
$$


So assuming that $q \sim_{t} q^{\prime}$, we have to show that

$$
\left.\lambda x . e\left[\left\{q_{1} \ldots q_{n}\right\} /\left\{x_{1} \ldots x_{n}\right\}\right]\right) q \sim_{t} \Omega q^{\prime}
$$

which by Lemma 6.6 and Lemma 6.4 amounts to showing that

$$
\left.e\left[\left\{q, q_{1} \ldots q_{n}\right\} /\left\{x, x_{1} \ldots x_{n}\right\}\right]\right) \sim_{t} \Omega
$$

But this follows from $e \operatorname{COR}\left(t,(0, W),{ }_{-},-\right) e^{\prime}$.

- Suppose $\Gamma, T \vdash_{s a} \lambda x . e: t_{1} \rightarrow_{1} t, W$ because $\left(\left(x: t_{1}\right), \Gamma\right), T \cup\{x\} \vdash_{s a}$ $e: t,(1, W)$. Here $\lambda$ x.e translates into $\lambda x . e^{\prime}$, where $e$ translates into $e^{\prime}$.

Our first task is to show that $Z_{T}(\Gamma) \vdash \lambda x \cdot e^{\prime}: Z\left(t_{1} \rightarrow_{1} t\right)$. This can be done by showing that $\left(\left(x:\left[Z\left(t_{1}\right)\right]\right), Z_{T}(\Gamma)\right) \vdash e^{\prime}: Z(t)$ but as $\left(\left(x:\left[Z\left(t_{1}\right)\right]\right), Z_{T}(\Gamma)\right)=Z_{T \cup\{x\}}\left(\left(x: t_{1}\right), \Gamma\right)$ this follows from $e$ $\operatorname{COR}\left(t,,_{-,},-\right) e^{\prime}$.

Now suppose $q_{i} \sim_{\Gamma\left(x_{i}\right)} q_{i}^{\prime}$. We have to show that

$$
\lambda x .\left(e\left[\left\{q_{1} \ldots q_{n}\right\} /\left\{x_{1} \ldots x_{n}\right\}\right]\right) \sim_{t_{1} \rightarrow_{0} t} \lambda x .\left(e^{\prime}\left[\left\{Q_{1}^{\prime} \ldots Q_{n}^{\prime}\right\} /\left\{x_{1} \ldots x_{n}\right\}\right]\right)
$$

Assuming that $q \sim_{t_{1}} q^{\prime}$, this amounts to showing

$$
\lambda x .\left(e\left[\left\{q_{1} \ldots q_{n}\right\} /\left\{x_{1} \ldots x_{n}\right\}\right]\right) q \sim_{t} \lambda x .\left(e^{\prime}\left[\left\{Q_{1}^{\prime} \ldots Q_{n}^{\prime}\right\} /\left\{x_{1} \ldots x_{n}\right\}\right]\right) \underline{q}^{\prime}
$$

which (by Lemma 6.6) amounts to showing that

$$
\left.e\left[\left\{q, q_{1} \ldots q_{n}\right\} /\left\{x, x_{1} \ldots x_{n}\right\}\right] \sim_{t} e^{\prime}\left[\left\{\underline{q^{\prime}}, Q_{1}^{\prime} \ldots Q_{n}^{\prime}\right\} /\left\{x, x_{1} \ldots x_{n}\right\}\right]\right)
$$

But this follows from $e \operatorname{COR}\left(t,,_{-}, T \cup\{x\}\right) e^{\prime}$.

Finally, we have to show that if $W\left(x_{i}\right)=0, q_{i} \sim_{\Gamma\left(x_{i}\right)} \Omega$ then

$$
\left.\lambda x . e\left[\left\{q_{1} \ldots q_{n}\right\} /\left\{x_{1} \ldots x_{n}\right\}\right]\right) \sim_{t_{1} \rightarrow 1^{t}} \Omega
$$

So assuming that $q \sim_{t} q^{\prime}$, we have to show that

$$
\left.\lambda x . e\left[\left\{q_{1} \ldots q_{n}\right\} /\left\{x_{1} \ldots x_{n}\right\}\right]\right) q \sim_{t} \Omega \underline{q^{\prime}}
$$


which by Lemma 6.6 and Lemma 6.4 amounts to showing that

$$
\left.e\left[\left\{q, q_{1} \ldots q_{n}\right\} /\left\{x_{1} \ldots x_{n}\right\}\right]\right) \sim_{t} \Omega
$$

But this follows from $e \operatorname{COR}\left(t, W,_{-},,_{-}\right) e^{\prime}$.

- Suppose $\Gamma, T \vdash_{s a} e_{1} e_{2}: t_{1}, W$ because $\Gamma, T \vdash_{s a} e_{1}: t_{2} \rightarrow_{0} t_{1}, W_{1}$ and $\Gamma, T \vdash_{s a} e_{2}: t_{2}, W_{2}$ with $W(x)=0$ iff $W_{1}(x)=0$ or $W_{2}=0$. Here $e_{1} e_{2}$ translates into $e_{1}^{\prime} e_{2}^{\prime}$, where $e_{1}$ translates into $e_{1}^{\prime}$ and $e_{2}$ translates into $e_{2}^{\prime}$.

Our first task is to show that $Z_{T}(\Gamma) \vdash e_{1}^{\prime} e_{2}^{\prime}: Z\left(t_{1}\right)$. But as $e_{1}^{\prime}$ and $e_{2}^{\prime}$ are correct translations, we have $Z_{T}(\Gamma) \vdash e_{1}^{\prime}: Z\left(t_{2} \rightarrow_{0} t_{1}\right)$ and $Z_{T}(\Gamma) \vdash e_{2}^{\prime}: Z\left(t_{2}\right)$ enabling us to conclude the desired.

Next we must show that if $q_{i} \sim_{\Gamma\left(x_{i}\right)} q_{i}^{\prime}$ then

$$
\left.\left(e_{1} e_{2}\right)\left[\left\{q_{1} \ldots q_{n}\right\} /\left\{x_{1} \ldots x_{n}\right\}\right]\right) \sim_{t_{1}}\left(e_{1}^{\prime} e_{2}^{\prime}\right)\left[\left\{Q_{1}^{\prime} \ldots Q_{n}^{\prime}\right\} /\left\{x_{1} \ldots x_{n}\right\}\right]
$$

But this follows from $e_{1}$ and $e_{2}$ being correct translations, since

$$
\begin{gathered}
\left.e_{1}\left[\left\{q_{1} \ldots q_{n}\right\} /\left\{x_{1} \ldots x_{n}\right\}\right]\right) \sim_{t_{2} \rightarrow_{0} t_{1}} e_{1}^{\prime}\left[\left\{Q_{1}^{\prime} \ldots Q_{n}^{\prime}\right\} /\left\{x_{1} \ldots x_{n}\right\}\right] \text { and } \\
\left.e_{2}\left[\left\{q_{1} \ldots q_{n}\right\} /\left\{x_{1} \ldots x_{n}\right\}\right]\right) \sim_{t_{2}} e_{2}^{\prime}\left[\left\{Q_{1}^{\prime} \ldots Q_{n}^{\prime}\right\} /\left\{x_{1} \ldots x_{n}\right\}\right]
\end{gathered}
$$

Finally we must show that if $W\left(x_{i}\right)=0, q_{i} \sim_{\Gamma(i)} \Omega$ then $e_{1} e_{2}\left[\left\{q_{1} \ldots q_{n}\right\} /\left\{x_{1} \ldots x_{n}\right\}\right] \sim_{t_{1}} \Omega$. We distinguish between two cases:

- if $W_{1}\left(x_{i}\right)=0$, then $\left(\operatorname{as} e_{1} \operatorname{COR}\left({ }_{-}, W_{1},{ }_{-},{ }_{-}\right) e_{1}^{\prime}\right)$ it holds that $e_{1}\left[\left\{q_{1} \ldots q_{n}\right\} /\left\{x_{1} \ldots x_{n}\right\}\right] \sim_{t_{2} \rightarrow_{0} t_{1}} \Omega$. As $e_{2} \operatorname{COR}\left(,_{-},,_{-},{ }_{-}\right) e_{2}^{\prime}$, we can conclude

$$
\begin{gathered}
e_{1}\left[\left\{q_{1} \ldots q_{n}\right\} /\left\{x_{1} \ldots x_{n}\right\}\right] e_{2}\left[\left\{q_{1} \ldots q_{n}\right\} /\left\{x_{1} \ldots x_{n}\right\}\right] \sim_{t_{1}} \\
\Omega e_{2}\left[\left\{q_{1} \ldots q_{n}\right\} /\left\{x_{1} \ldots x_{n}\right\}\right]
\end{gathered}
$$

which (by Lemma 6.4) gives the desired.

- if $W_{2}\left(x_{i}\right)=0$, then $\left(\operatorname{as} e_{2} \operatorname{COR}\left({ }_{-}, W_{2},{ }_{-},{ }_{-}\right) e_{2}^{\prime}\right)$ it holds that $e_{2}\left[\left\{q_{1} \ldots q_{n}\right\} /\left\{x_{1} \ldots x_{n}\right\}\right] \sim_{t_{2}} \Omega$ and hence $\left(\operatorname{as} e_{1} \operatorname{COR}\left(,_{-},,_{-},-\right) e_{1}^{\prime}\right)$ $e_{1}\left[\left\{q_{1} \ldots q_{n}\right\} /\left\{x_{1} \ldots x_{n}\right\}\right] e_{2}\left[\left\{q_{1} \ldots q_{n}\right\} /\left\{x_{1} \ldots x_{n}\right\}\right] \sim_{t_{1}}$ $e_{1}^{\prime}\left[\left\{Q_{1}^{\prime} \ldots Q_{n}^{\prime}\right\} /\left\{x_{1} \ldots x_{n}\right\}\right] \Omega$ 
As $e_{1}^{\prime}\left[\left\{Q_{1}^{\prime} \ldots Q_{n}^{\prime}\right\} /\left\{x_{1} \ldots x_{n}\right\}\right] \Omega$ loops by $\Rightarrow_{V}$, Lemma 6.4 gives the desired result.

- Suppose $\Gamma, T \vdash_{s a} e_{1} e_{2}: t_{1}, W_{1}$ because $\Gamma, T \vdash_{s a} e_{1}: t_{2} \rightarrow_{1} t_{1}, W_{1}$ and $\Gamma, T \vdash_{s a} e_{2}: t_{2}, W_{2}$. Here $e_{1} e_{2}$ translates into $e_{1}^{\prime} \underline{e_{2}^{\prime}}$, where $e_{1}$ translates into $e_{1}^{\prime}$ and $e_{2}$ translates into $e_{2}^{\prime}$. Inductively, we can assume that

$e_{1} \operatorname{COR}\left(t_{2 \rightarrow 1} t_{1}, W_{1}, \Gamma, T\right) e_{1}^{\prime}$ and $e_{2} \operatorname{COR}\left(t_{2}, W_{2}, \Gamma, T\right) e_{2}^{\prime}$.

Our first task is to show that $Z_{T}(\Gamma) \vdash e_{1}^{\prime} e_{2}^{\prime}: Z\left(t_{1}\right)$. But as $e_{1}^{\prime}$ and $e_{2}^{\prime}$ are correct translations, we have $Z_{T}\left(\overline{\Gamma)} \vdash e_{1}^{\prime}: Z\left(t_{2} \rightarrow_{0} t_{1}\right)=\right.$ $\left[Z\left(t_{2}\right)\right] \rightarrow Z\left(t_{1}\right)$ and $Z_{T}(\Gamma) \vdash e_{2}^{\prime}: Z\left(t_{2}\right)$ enabling us to conclude the desired.

Next we must show that if $q_{i} \sim_{\Gamma\left(x_{i}\right)} q_{i}$ then

$$
\left(e_{1} e_{2}\right)\left[\left\{q_{1} \ldots q_{n}\right\} /\left\{x_{1} \ldots x_{n}\right\}\right] \sim_{t_{1}}\left(e_{1}^{\prime} \underline{e_{2}^{\prime}}\right)\left[\left\{Q_{1}^{\prime} \ldots Q_{n}^{\prime}\right\} /\left\{x_{1} \ldots x_{n}\right\}\right]
$$

But this follows easily from $e_{1}^{\prime}$ and $e_{2}^{\prime}$ being correct translations.

Finally we must show that if $W_{1}\left(x_{i}\right)=0, q_{i} \sim_{\Gamma(i)} \Omega$ then

$$
e_{1} e_{2}\left[\left\{q_{1} \ldots q_{n}\right\} /\left\{x_{1} \ldots x_{n}\right\}\right] \sim_{t_{1}} \Omega
$$

As $e_{1} \operatorname{COR}\left({ }_{-}, W_{1},,_{-},-\right) e_{1}^{\prime}$ we have $e_{1}\left[\left\{q_{1} \ldots q_{n}\right\} /\left\{x_{1} \ldots x_{n}\right\} \sim_{t_{2} \rightarrow 1} t_{1} \Omega\right.$.

As $e_{2} \operatorname{COR}\left(,_{-},,_{-},{ }_{-}\right) e_{2}^{\prime}$, we can conclude

$$
\begin{gathered}
\left.e_{1}\left[\left\{q_{1} \ldots q_{n}\right\} /\left\{x_{1} \ldots x_{n}\right\}\right] e_{2}\left[\left\{q_{1} \ldots q_{n}\right\} /\left\{x_{1} \ldots x_{n}\right\}\right]\right] \\
\Omega \underline{e}_{2}^{\prime}\left[\left\{Q_{1}^{\prime} \ldots Q_{n}^{\prime}\right\} /\left\{x_{1} \ldots x_{n}\right\}\right]
\end{gathered} \sim_{t_{1}}
$$

which (by Lemma 6.4) gives the desired.

- Suppose $\Gamma, T \vdash_{s a}$ if $e_{1} e_{2} e_{3}: t, W$ because $\Gamma, T \vdash_{s a} e_{1}$ Bool, $W_{1}, \Gamma, T \vdash_{s a}$ $e_{2}: t, W_{2}, \Gamma, T \vdash_{s a} e_{3}: t, W_{3}$ and $W(x)=W_{1}(x) \sqcap\left(W_{2}(x) \sqcup W_{3}(x)\right)$. Now if $e_{1} e_{2} e_{3}$ translates into if $e_{1}^{\prime} e_{2}^{\prime} e_{3}^{\prime}$, where $e_{i}$ translates into $e_{i}^{\prime}$.

Our first task is to show that $Z_{T}(\Gamma) \vdash$ if $e_{1}^{\prime} e_{2}^{\prime} e_{3}^{\prime}: Z(t)$. But this is immediate, since the correctness of the $e_{i}^{\prime}$ 's tells us that

$Z_{T}(\Gamma) \vdash e_{1}^{\prime}: Z($ Bool $)=$ Bool and $Z_{T}(\Gamma) \vdash e_{2}^{\prime}: Z(t)$ and $Z_{T}(\Gamma) \vdash$ $e_{1}^{\prime}: Z(t)$

Next assume that $q_{i} \sim_{\Gamma\left(x_{i}\right)} q_{i}^{\prime}$, then we must show that 


$$
\begin{array}{rll} 
& \text { if } e_{1}\left[\left\{q_{1} \ldots q_{n}\right\} /\left\{x_{1} \ldots x_{n}\right\}\right] & e_{2}\left[\left\{q_{1} \ldots q_{n}\right\} /\left\{x_{1} \ldots x_{n}\right\}\right] \\
& e_{3}\left[\left\{q_{1} \ldots q_{n}\right\} /\left\{x_{1} \ldots x_{n}\right\}\right] \\
\sim_{t} \quad \text { if } e_{1}^{\prime}\left[\left\{Q_{1}^{\prime} \ldots Q_{n}^{\prime}\right\} /\left\{x_{1} \ldots x_{n}\right\}\right] & e_{2}^{\prime}\left[\left\{Q_{1}^{\prime} \ldots Q_{n}^{\prime}\right\} /\left\{x_{1} \ldots x_{n}\right\}\right] \\
& e_{3}^{\prime}\left[\left\{Q_{1}^{\prime} \ldots Q_{n}^{\prime}\right\} /\left\{x_{1} \ldots x_{n}\right\}\right]
\end{array}
$$

We distinguish between three cases:

$-e_{1}\left[\left\{q_{1} \ldots q_{n}\right\} /\left\{x_{1} \ldots x_{n}\right\}\right]$ loops $\left(\right.$ by $\left.\Rightarrow_{N}\right)$. Then (as $e_{1}^{\prime}$ is a correct translation of $e_{1}$ ) also $e_{1}^{\prime}\left[\left\{Q_{1}^{\prime} \ldots Q_{n}^{\prime}\right\} /\left\{x_{1} \ldots x_{n}\right\}\right]$ loops (by $\Rightarrow_{V}$ ). Now apply Lemma 6.5.

$-e_{1}\left[\left\{q_{1} \ldots q_{n}\right\} /\left\{x_{1} \ldots x_{n}\right\}\right] \Rightarrow_{N}^{*}$ True. Then also $e_{1}^{\prime}\left[\left\{Q_{1}^{\prime} \ldots Q_{n}^{\prime}\right\} /\left\{x_{1} \ldots x_{n}\right\}\right] \Rightarrow_{V}^{*}$ True. By repeated application of Lemma 6.6, it is enough to show that

$$
e_{2}\left[\left\{q_{1} \ldots q_{n}\right\} /\left\{x_{1} \ldots x_{n}\right\}\right] \sim_{t} e_{2}^{\prime}\left[\left\{Q_{1}^{\prime} \ldots Q_{n}^{\prime}\right\} /\left\{x_{1} \ldots x_{n}\right\}\right]
$$

but this follows from $e_{2}^{\prime}$ being a correct translation.

$-e_{1}\left[\left\{q_{1} \ldots q_{n}\right\} /\left\{x_{1} \ldots x_{n}\right\}\right] \Rightarrow_{N}^{*}$ False. This is analogous to the previous case.

Finally, we have to check that if $W\left(x_{i}\right)=0$ and $q_{i} \sim_{\Gamma\left(x_{i}\right)} \Omega$ then

$$
\begin{aligned}
& \text { if } e_{1}\left[\left\{q_{1} \ldots q_{n}\right\} /\left\{x_{1} \ldots x_{n}\right\}\right] \quad e_{2}\left[\left\{q_{1} \ldots q_{n}\right\} /\left\{x_{1} \ldots x_{n}\right\}\right] \\
& e_{3}\left[\left\{q_{1} \ldots q_{n}\right\} /\left\{x_{1} \ldots x_{n}\right\}\right] \sim_{t} \Omega
\end{aligned}
$$

We split between two cases:

$-W_{1}\left(x_{i}\right)=0$. Then (as $e_{1} \operatorname{COR}\left(\right.$ Bool, $\left.\left.W_{1},{ }_{-},{ }_{-}\right) e_{1}^{\prime}\right)$ it holds that $e_{1}\left[\left\{q_{1} \ldots q_{n}\right\} /\left\{x_{1} \ldots x_{n}\right\}\right] \sim_{\text {Bool }} \Omega$ which amounts to saying that $e_{1}\left[\left\{q_{1} \ldots q_{n}\right\} /\left\{x_{1} \ldots x_{n}\right\}\right]$ loops - hence the claim.

$-W_{2}\left(x_{i}\right)=0$ and $W_{3}\left(x_{i}\right)=0$. Then $\left(\operatorname{as} e_{2} \operatorname{COR}\left(t, W_{2},{ }_{-},{ }_{-}\right) e_{2}^{\prime}\right.$ and $\left.e_{3} \operatorname{COR}\left(t, W_{3},{ }_{-},{ }_{-}\right)\right)$

$e_{2}\left[\left\{q_{1} \ldots q_{n}\right\} /\left\{x_{1} \ldots x_{n}\right\}\right] \sim_{t} \Omega$ and $e_{3}\left[\left\{q_{1} \ldots q_{n}\right\} /\left\{x_{1} \ldots x_{n}\right\}\right] \sim_{t} \Omega$ If $e_{1}\left[\left\{q_{1} \ldots q_{n}\right\} /\left\{x_{1} \ldots x_{n}\right\}\right] \Rightarrow_{N}^{*}$ True, the claim follows from the above and Lemma 6.6. Similarly in the False-case.

If $e_{1}\left[\left\{q_{1} \ldots q_{n}\right\} /\left\{x_{1} \ldots x_{n}\right\}\right]$ loops, the claim is trivial. 
- Suppose $\Gamma, T \vdash_{s a} \operatorname{rec}_{n} f e: t, W$ because $((f: t), \Gamma), T \vdash_{s a} e$ : $t,(b, W)$. Now $\operatorname{rec}_{n} f$ e translates into $\operatorname{rec}_{n} f e^{\prime}$, where $e^{\prime}$ is the translation of $e$.

First we have to check that $Z_{T}(\Gamma) \vdash \operatorname{rec}_{n} f e^{\prime}: Z(t)$ but this is immediate since the correctness of $e^{\prime}$ tells us that $\left((f: Z(t)), Z_{T}(\Gamma)\right) \vdash$ $e^{\prime}: Z(t)$.

Next assume that $q_{i} \sim_{\Gamma\left(x_{i}\right)} q_{i}^{\prime}$, then we have to show that

$$
\operatorname{rec}_{n} f e\left[\left\{q_{1} \ldots q_{n}\right\} /\left\{x_{1} \ldots x_{n}\right\}\right] \sim_{t} \operatorname{rec}_{n} f e^{\prime}\left[\left\{Q_{1}^{\prime} \ldots Q_{n}^{\prime}\right\} /\left\{x_{1} \ldots x_{n}\right\}\right]
$$

This follows from Lemma 6.8, provided we can show that

$$
\begin{aligned}
& q \sim_{t} q^{\prime} \text { implies that } \\
& e\left[\left\{q, q_{1} \ldots q_{n}\right\} /\left\{f, x_{1} \ldots x_{n}\right\}\right] \sim_{t} e^{\prime}\left[\left\{q^{\prime}, Q_{1}^{\prime} \ldots Q_{n}^{\prime}\right\}\left\{f, x_{1} \ldots x_{n}\right\}\right]
\end{aligned}
$$

But this follows from $e^{\prime}$ being a correct translation of $e$.

Finally, we have to show that if $W\left(x_{i}\right)=0$ and $q_{i} \sim_{\Gamma\left(x_{i}\right)} \Omega$ then

$$
\operatorname{rec}_{n} f e\left[\left\{q_{1} \ldots q_{n}\right\} /\left\{x_{1} \ldots x_{n}\right\}\right] \sim_{t} \Omega
$$

This follows from Lemma 6.9, provided we can show that if $q \sim_{t} q^{\prime}$ then

$$
e\left[\left\{q, q_{1} \ldots q_{n}\right\} /\left\{f, x_{1} \ldots x_{n}\right\}\right] \sim_{t} \Omega
$$

But this follows from $e^{\prime}$ being a correct translation of $e$.

This concludes the proof of Theorem 6.2.

\section{Concluding Remarks}

We believe the main contributions of this paper to be:

- one more (the second, the first being [Wan93]) application has been given of the paradigm: an analysis and a transformation exploiting this analysis should be proved correct simultaneously; 
- a (we think) novel approach to constraint solving, based on normalizing constraints while distinguishing between co/contravariant polarity, has been presented.

ln order to give a more precise analysis one may consider annotating the function arrows with the free variables needed by the function, cf. Example 3.6.

And to avoid the kind of superfluous dethunkification/thunkification we encountered in Example 5.2, one may consider keeping track of context somewhat similar to what is done in [NN90].

\section{References}

[BHA86] Geoffrey L. Burn, Chris Hankin, and Samson Abramsky. Strictness analysis for higher-order functions. Science of Computer Programming, 7:249-278, 1986.

[DH92] Olivier Danvy and John Hatcliff. Thunks (continued). In M. Billaud et al., editor, Analyse statique, Bordeaux 92 (WSA '92), pages 3-11, September 1992.

[DH93] Olivier Danvy and John Hatcliff. CPS transformation after strictness analysis. ACM Letters on Programming Languages and Systems, 1(3), 1993.

[Hug89] John Hughes. Why functional programming matters. Computer Journal, 32(2):98-107, 1989.

[Jen91] Thomas P. Jensen. Strictness analysis in logical form. In John Hughes, editor, International Conference on Functional Programming Languages and Computer Architecture, pages 352-366. Springer Verlag, LNCS 523, August 1991.

[KM89] Tsung-Min Kuo and Prateek Mishra. Strictness analysis: A new perspective based on type inference. In International Conference on Functional Programming Languages and Computer Architecture, pages 260272. ACM Press, September 1989. 
[Lan92] Torben Poort Lange. The correctness of an optimized code generation. Technical Report PB-427, DAIMI, University of Aarhus, Denmark, November 1992. Also in the proceedings of PEPM '93, Copenhagen, ACM press.

[Myc80] Alan Mycroft. The theory of transforming call-by-need to call-byvalue. In B. Robinet, editor, International Symposium on Programming, Paris, pages 269-281. Springer Verlag, LNCS 83, April 1980.

[NN90] Hanne Riis Nielson and Flemming Nielson. Context information for lazy code generation. In ACM Conference on Lisp and Functional Programming, pages 251-263. ACM Press, June 1990.

[Wan93] Mitchell Wand. Specifying the correctness of binding-time analysis. In ACM Symposium on Principles of Programming Languages, pages 137-143. ACM Press, January 1993.

[Wri91] David A. Wright. A new technique for strictness analysis. In TAPSOFT 91, pages 235-258. Springer Verlag, LNCS 494, April 1991.

[Wri92] David A. Wright. An intensional type discipline. Australian Computer Science Communications, 14, January 1992.

\section{A An Implementation}

Below we list the commented source code of a system implementing the type inference algorithm from Sect. 4 and the translation algorithm from Sect. 5. At the end we show some examples of the use of the system, including the examples given in the main text.

The author is not to be kept responsible for possible errors in the system... 
I| This file contains an implementation of

II the inference algorithm and the translation algorithm

II described in DAIMI PB-448 by Torben Amtoft:

II "Strictness Types: an Inference Algorithm and an Application"

II Main structure of the system:

II 0) the user supplies an expression where bound variables

II are annotated with their underlying types.

II Such an expression is represented as

II something of type "exptree"?

II 1) by means of the function "annotate" this expression

II is transformed into something of type "exptree_ta",

II i.e. an expression where all subexpressions are

II annotated with their types.

II This function is quite trivial.

II 2) by means of the function "infersolve" this expression

II is transformed into something of type "result_sa",

II i.e. an expression where all subexpressions are

II annotated with open strictness types,

II together with a list of normalized constraints

II among the strictness variables.

II This function implements the heart of the

II type inference algorithm in Section 4.

II 3) the user supplies the values of the strictness variables

II occurring in contravariant position in the overall type.

II Then the function "miniann" transforms the expression

II from 2) into something of type "exptree\_sta",

II i.e. an expression where all subexpressions are

II annotated with strictness types (and the minimal such).

II This function is rather trivial.

I| 4) by means of the function "translate" this expression

II is transformed into an expression of type "exptree", such

II that the CBV-semantics of this expression equals

II the CBN-semantics of the expression from 0 .

II This function implements the translation algorithm

II from Section 5 .

I| 5) by means of the functions "cbneval" and "cbveval"

II expressions of type "exptree" can be evaluated 


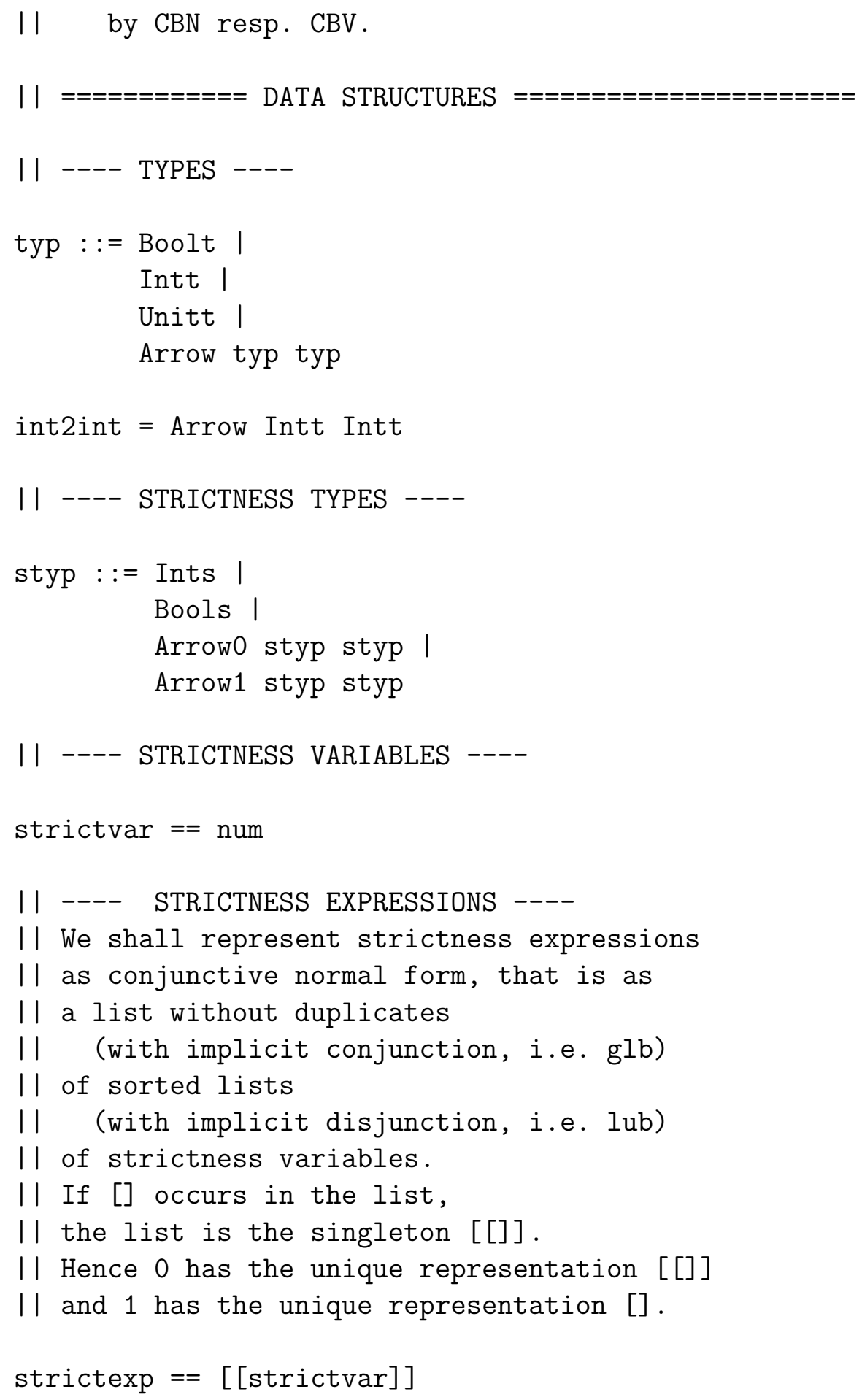




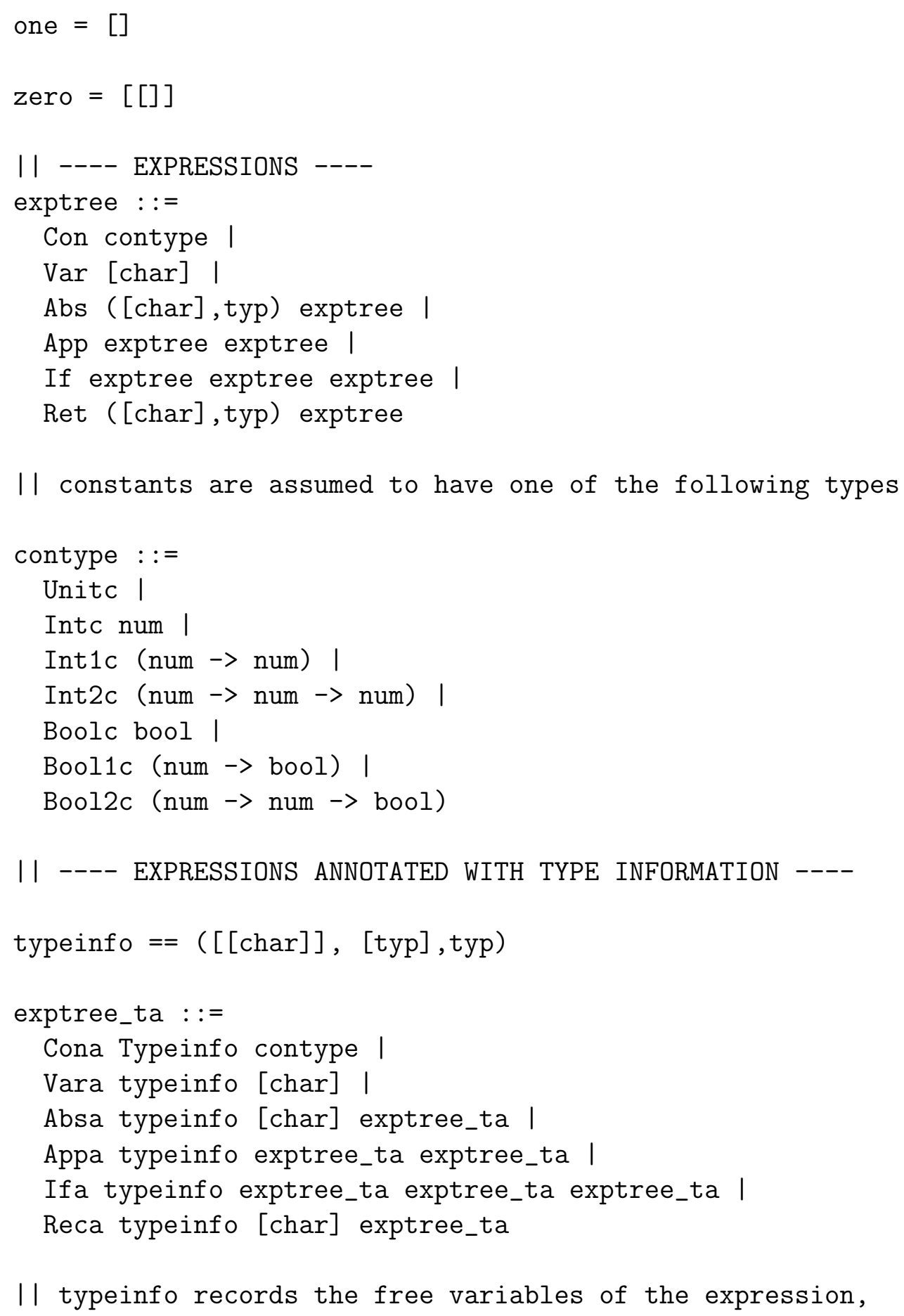


II their types and the type of the expression.

|| ---- EXPRESSIONS ANNOTATED WITH OPEN STRICTNESS TYPES

II TOGETHER WITH CONSTRAINTS ----

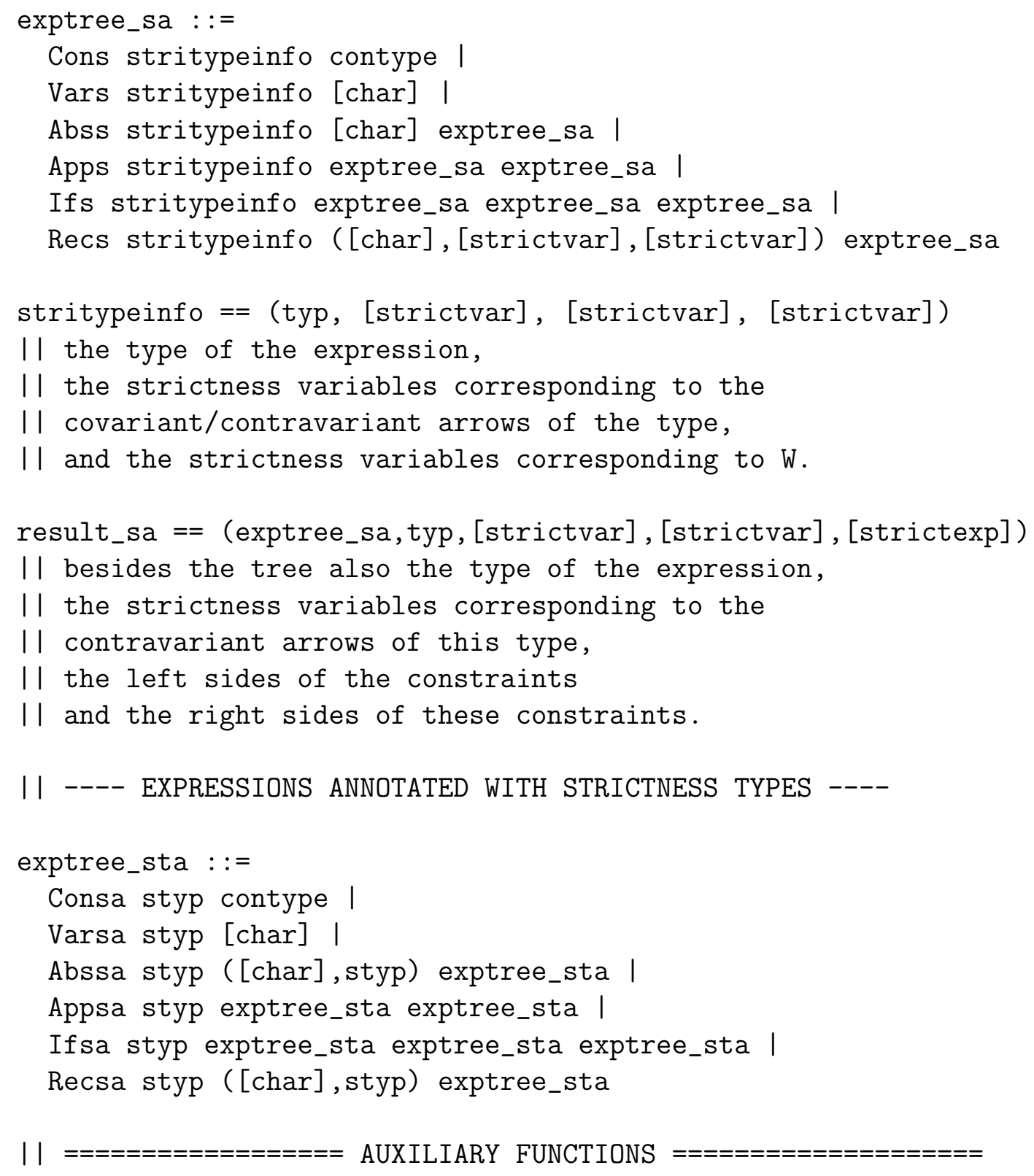




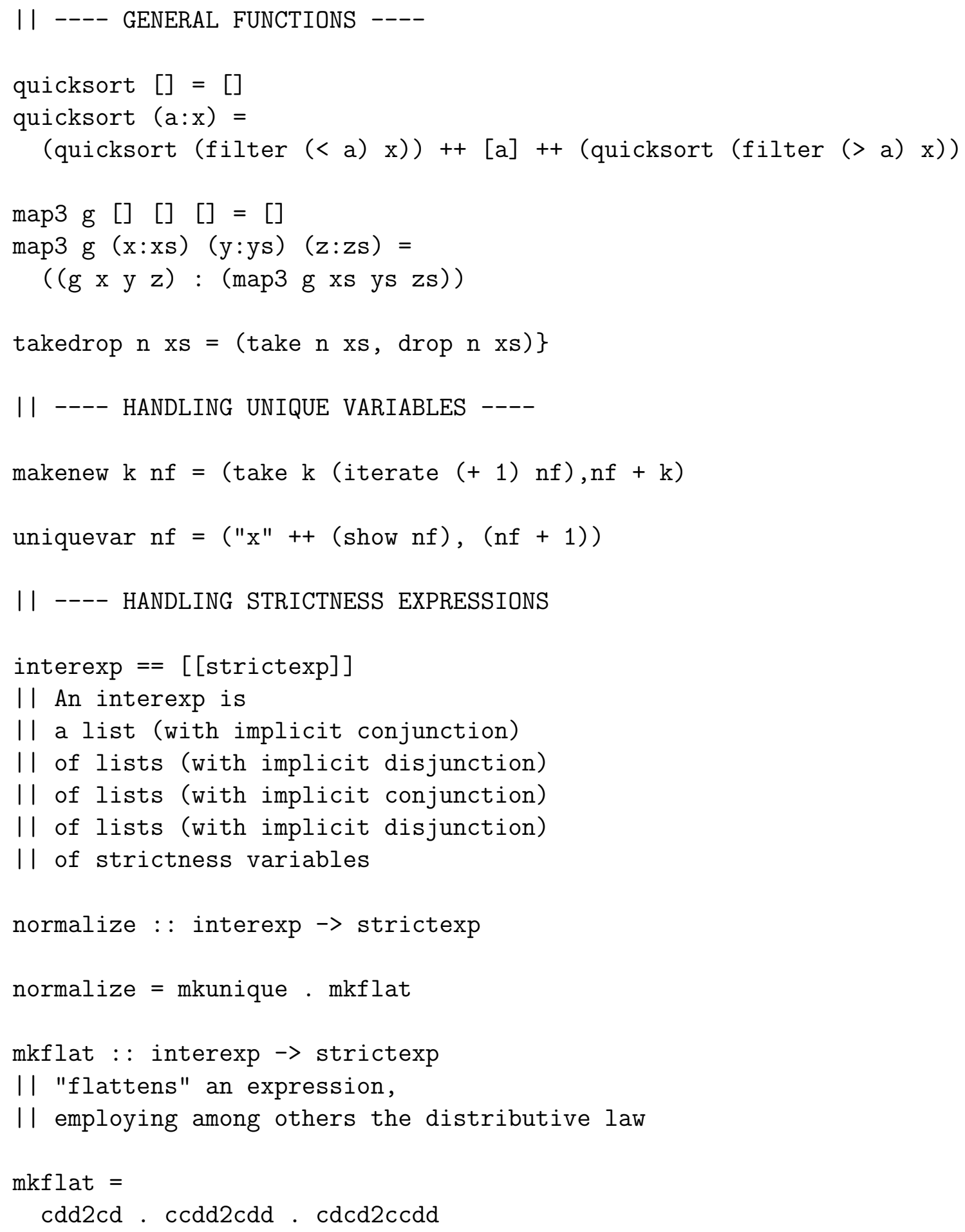




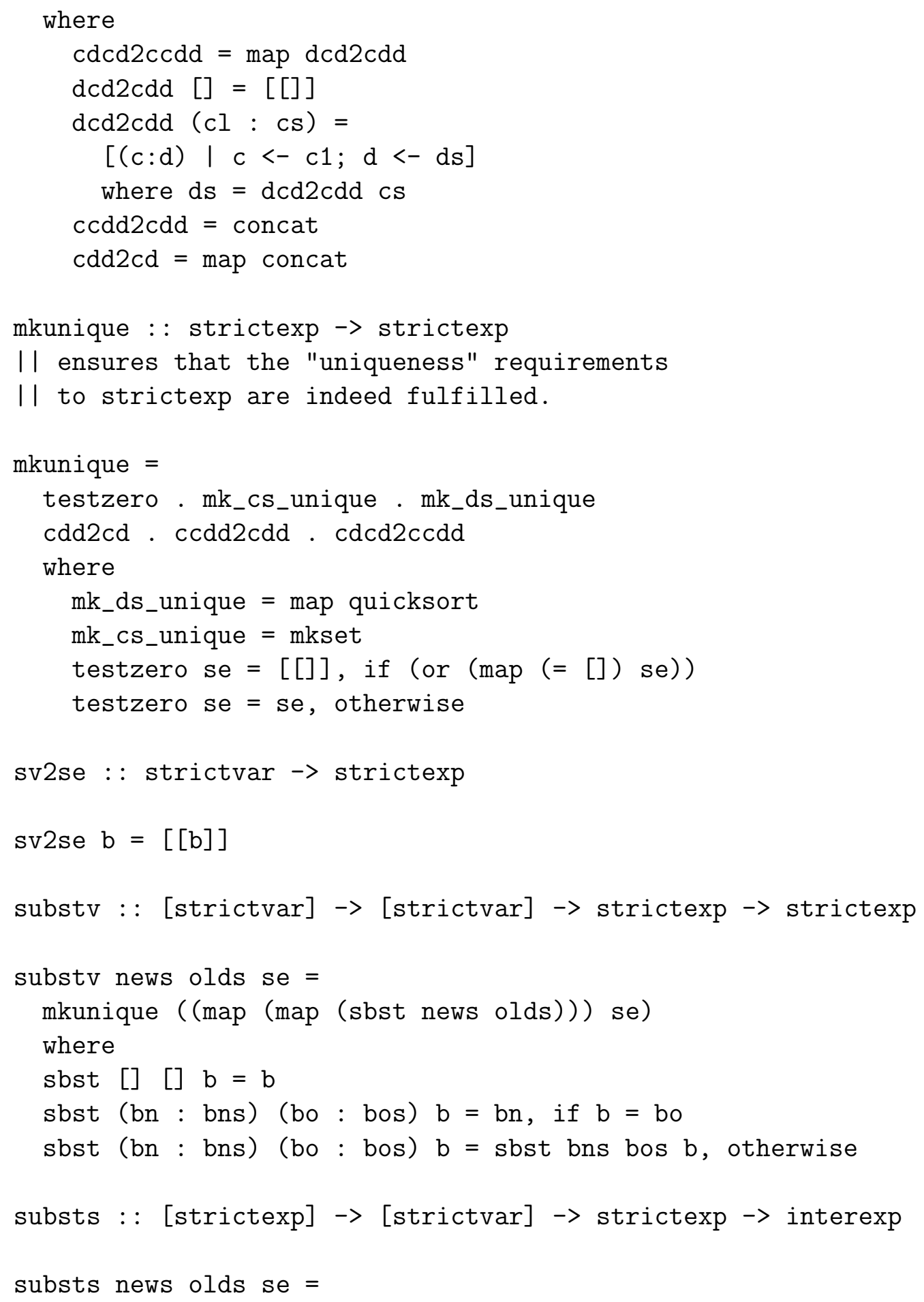




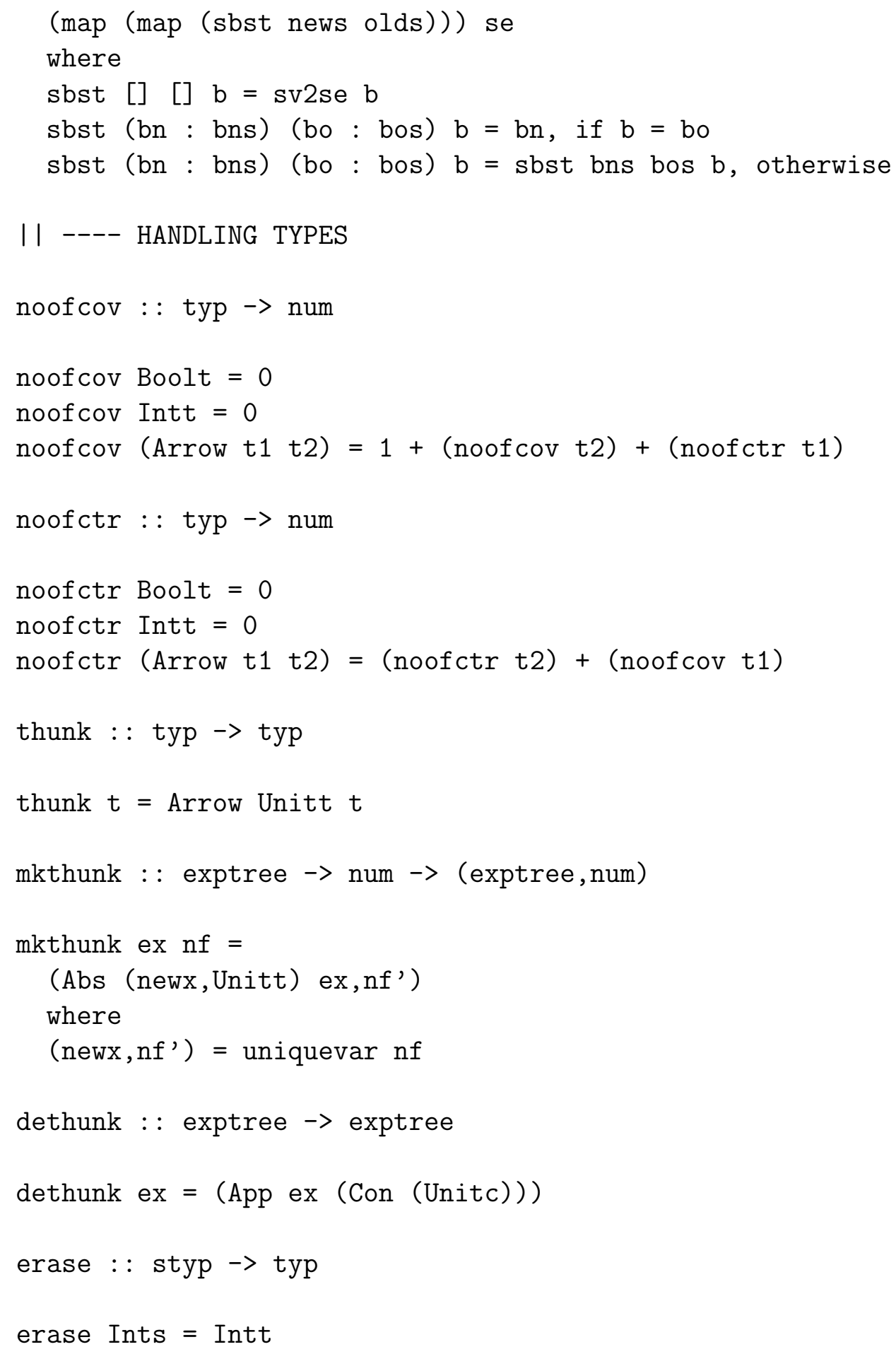




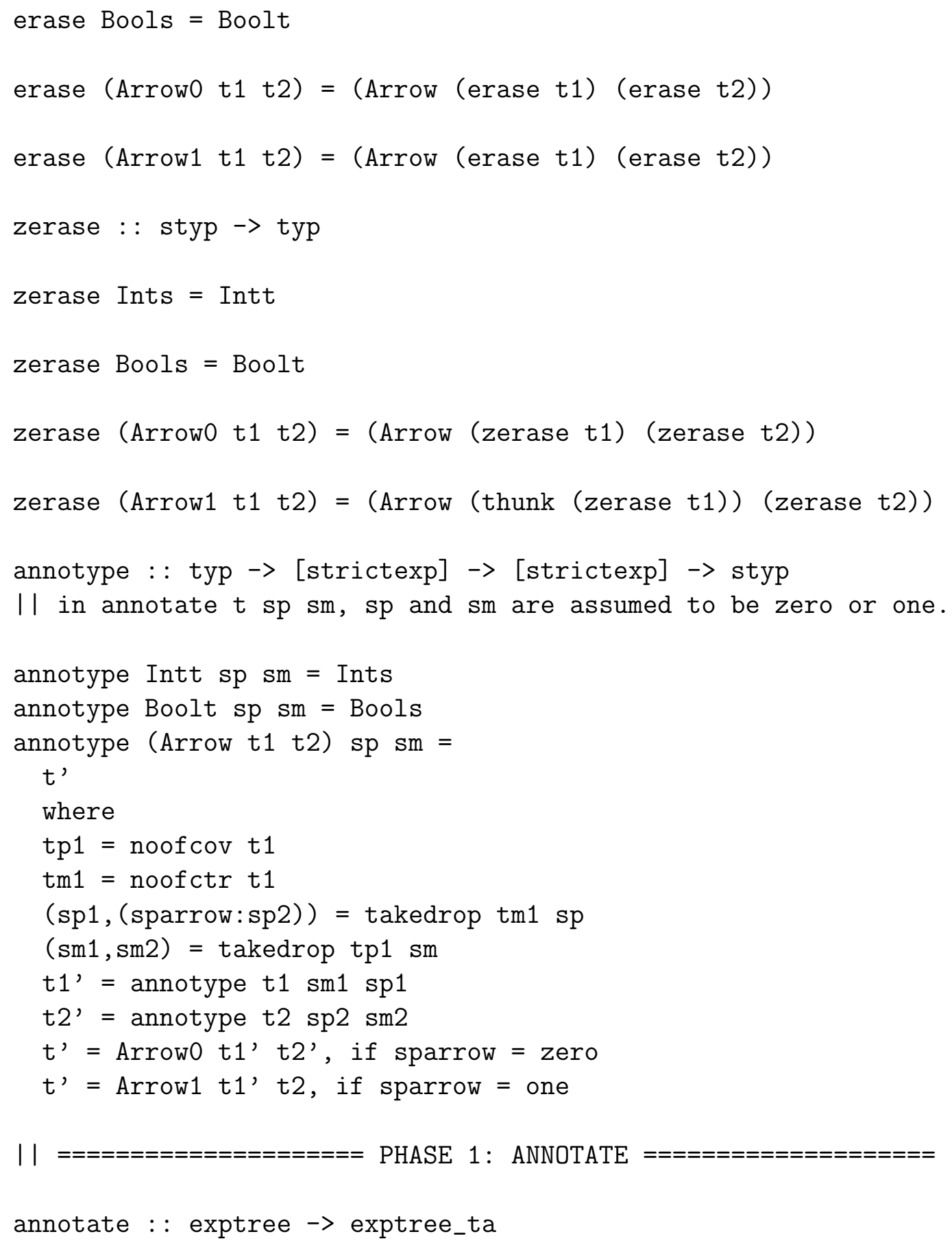




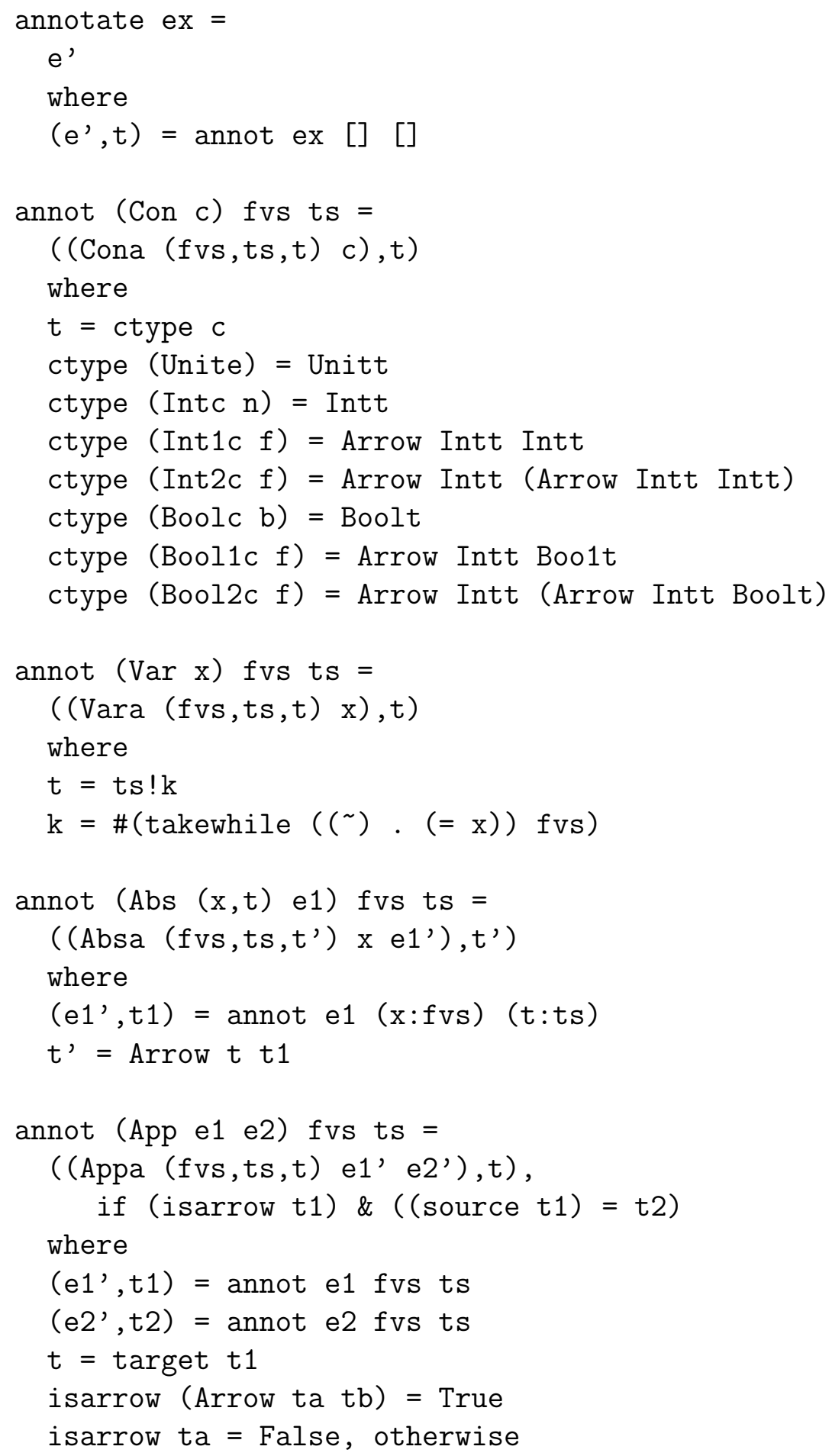




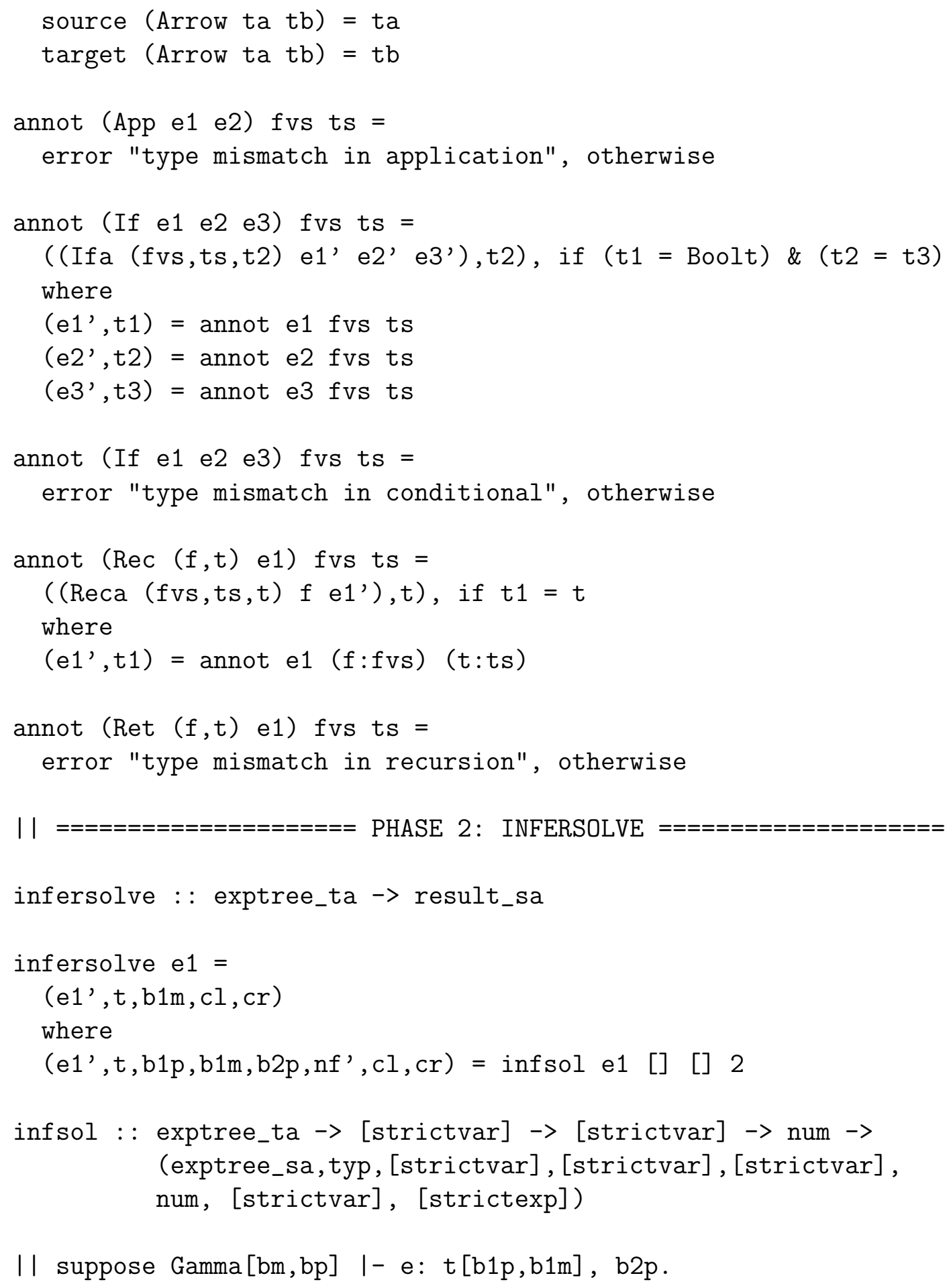


I| Then infsol e bp bm $n f=$ (e',t,b1p,b1m,b2p,nf', cl,cr)

II where nf records the next unused strictness variable, I| where $c l$ is a list of strictness variables

| 1 starting with $b p+b 1 p+b 2 p$,

II where $\mathrm{cr}$ is the corresponding list of strictness expressions

|| (so for the first ">=" is implicit

|| and for the remaining ">>" is implicit).

infsol (Cona (fvs,ts,t) c) bp bm nf =

((Cons (t,b1p, [],b2p) c), t,b1p, [],b2p,nf', , cl, cr)

where

tp $=$ noofcov $t$

$\left(\mathrm{b} 1 \mathrm{pnf} f^{\prime}\right)=$ makenew tp $\mathrm{nf}$

$\left(\mathrm{b} 2 \mathrm{p}, \mathrm{nf} \mathrm{f}^{\prime}\right)=$ makenew (\#fvs) nf'

$\mathrm{cl}=\mathrm{bp}++\mathrm{b} 1 \mathrm{p}++\mathrm{b} 2 \mathrm{p}$

$\mathrm{cr}=(\mathrm{rep}(\# \mathrm{bp})$ zero $)++$

(rep (\#b1p) zero) ++

(rep (\#b2p) one)

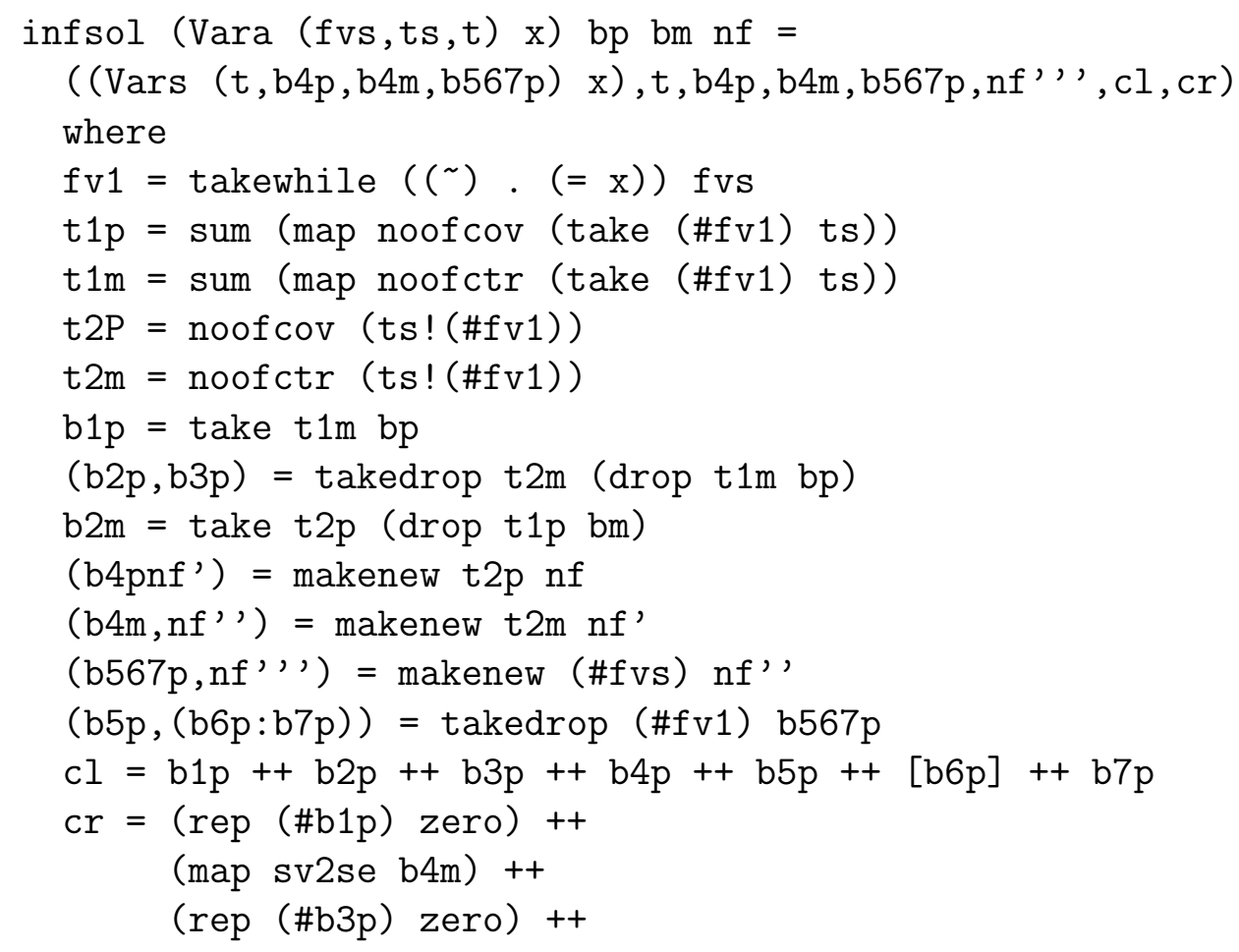




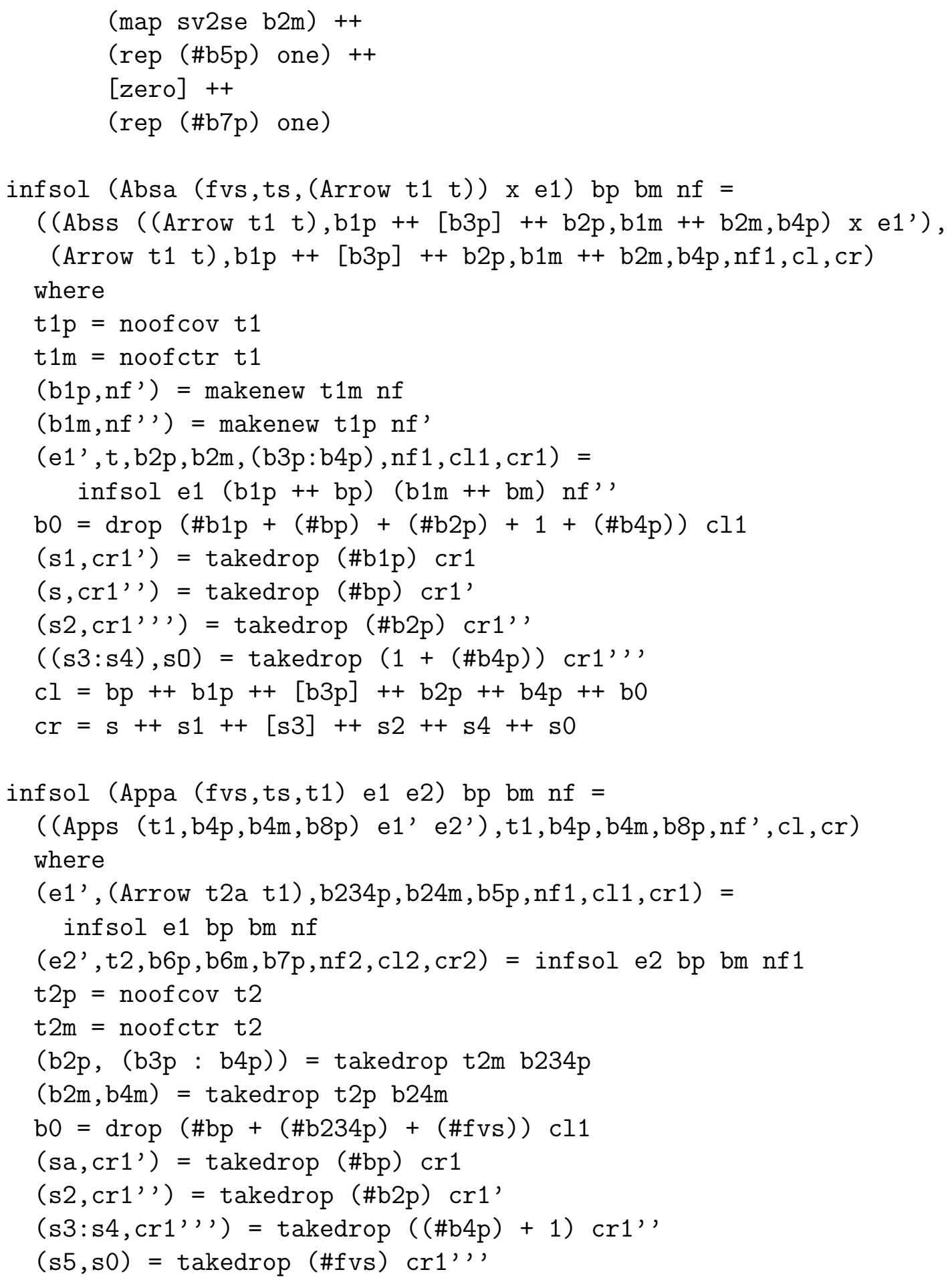




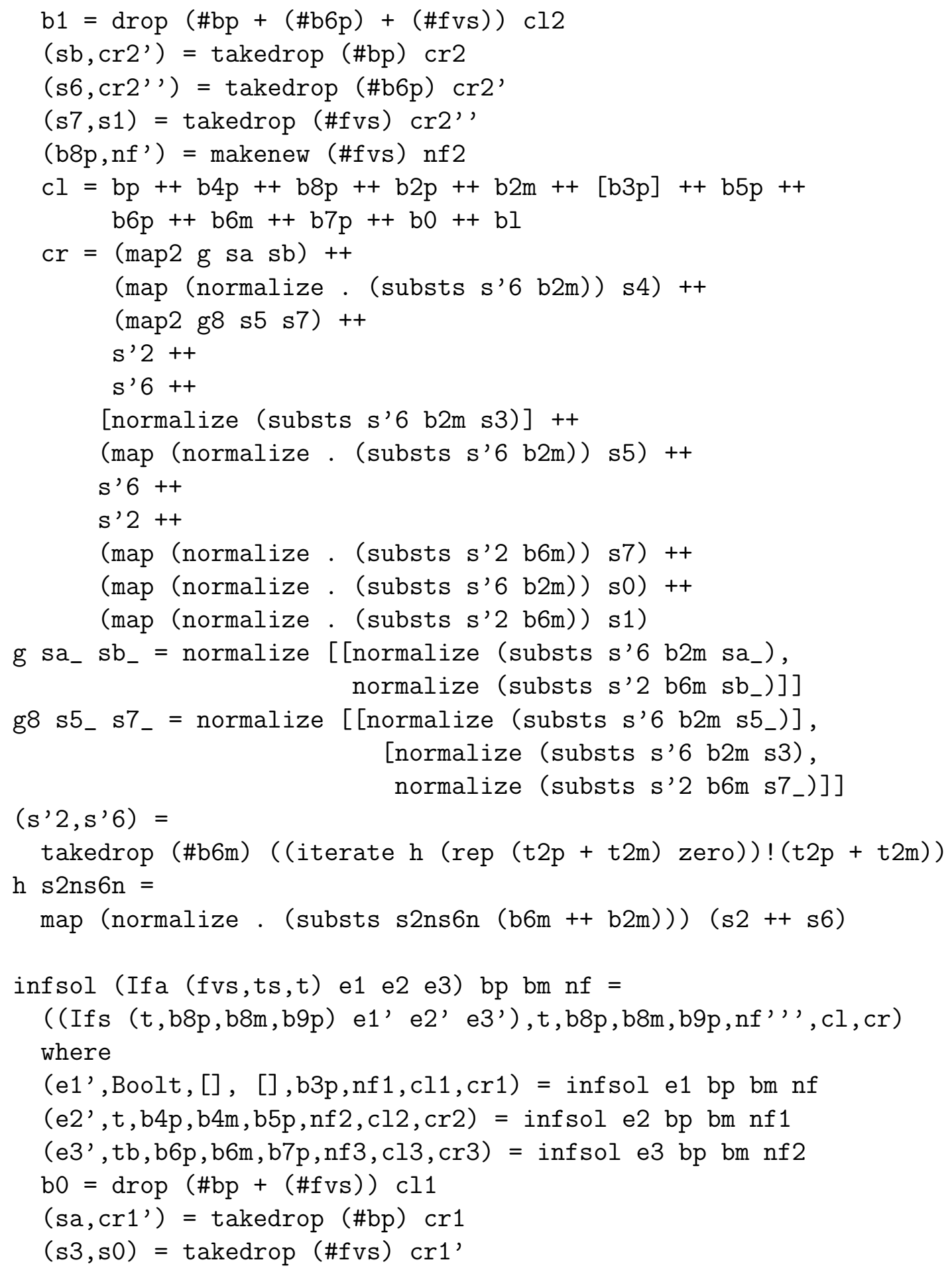




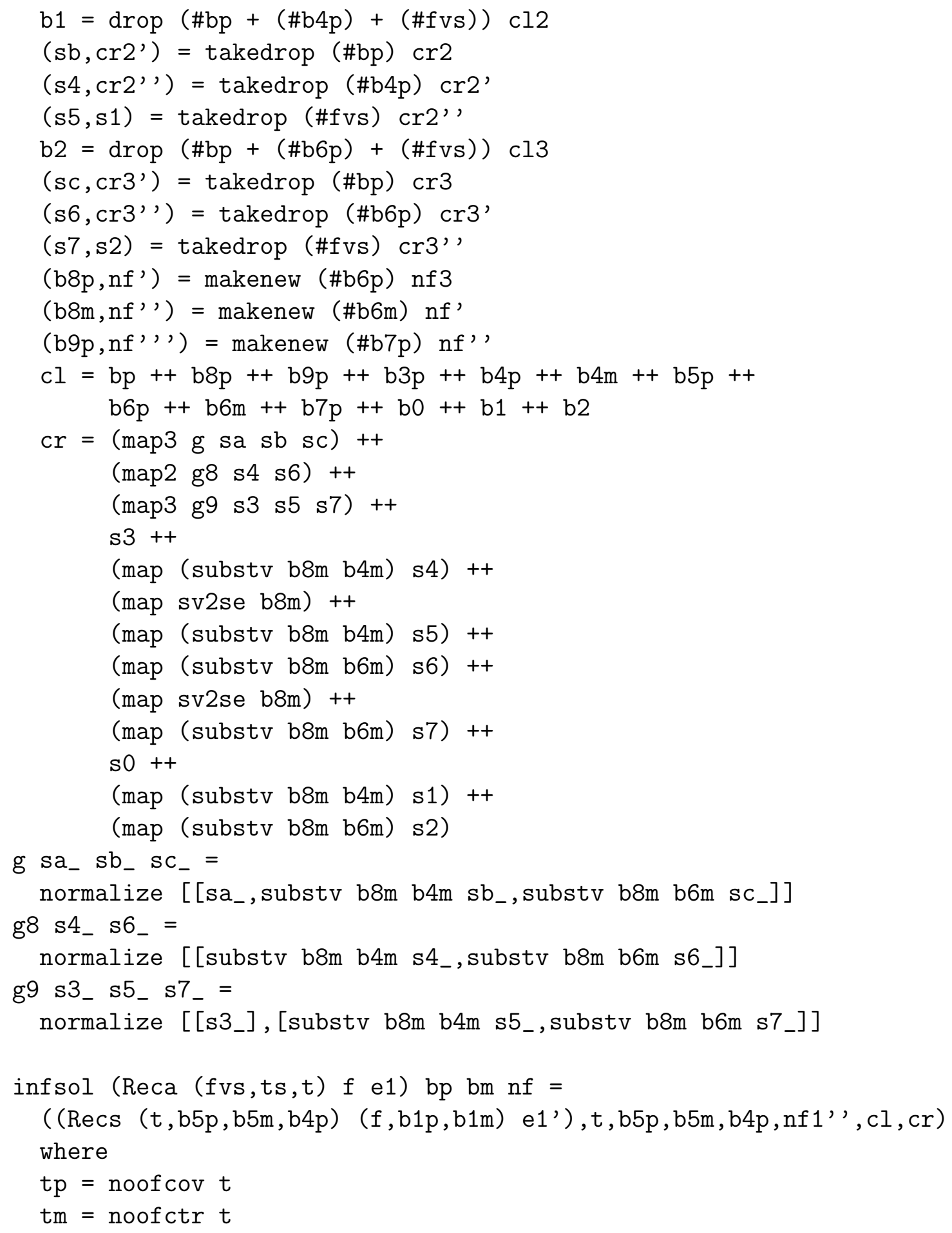




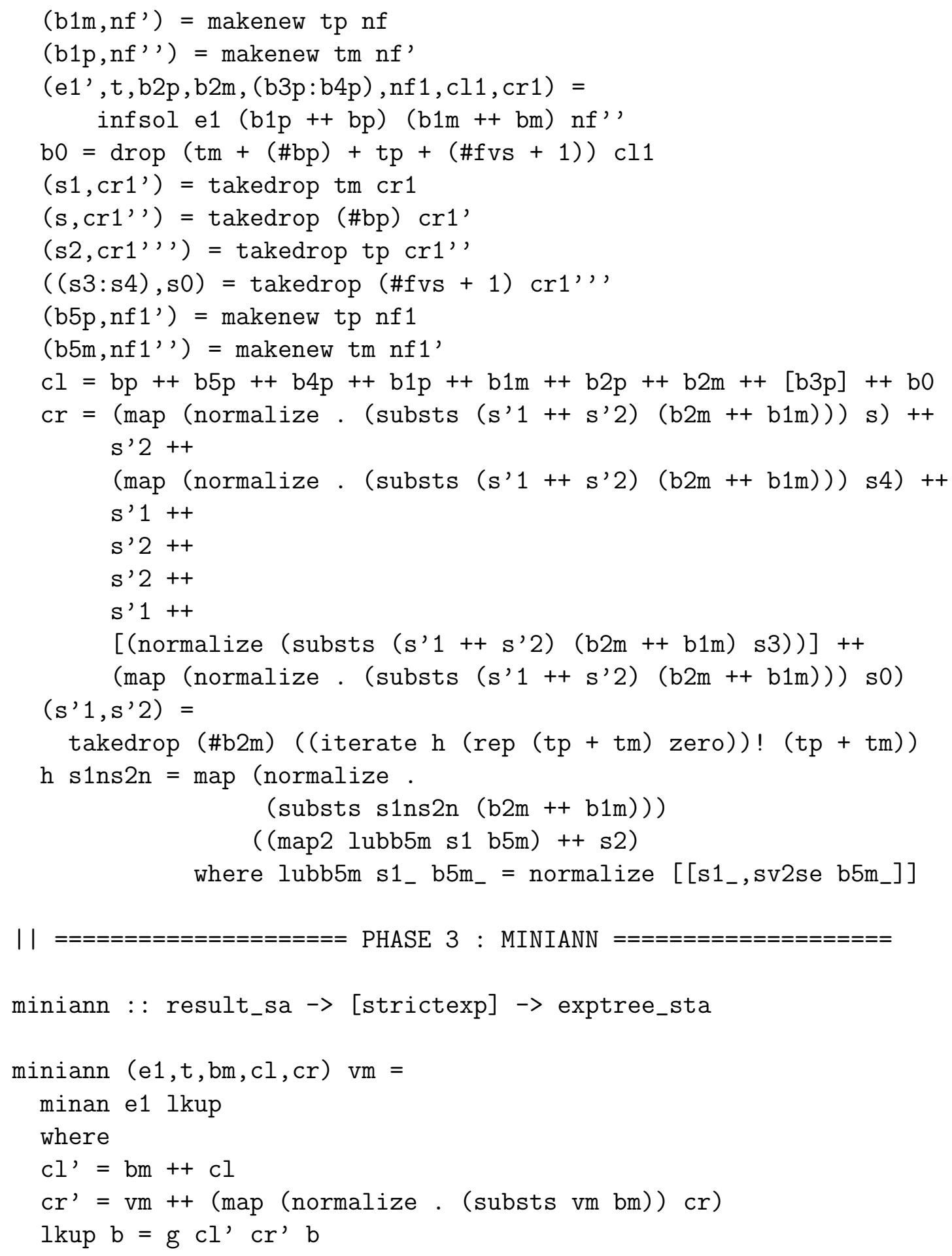




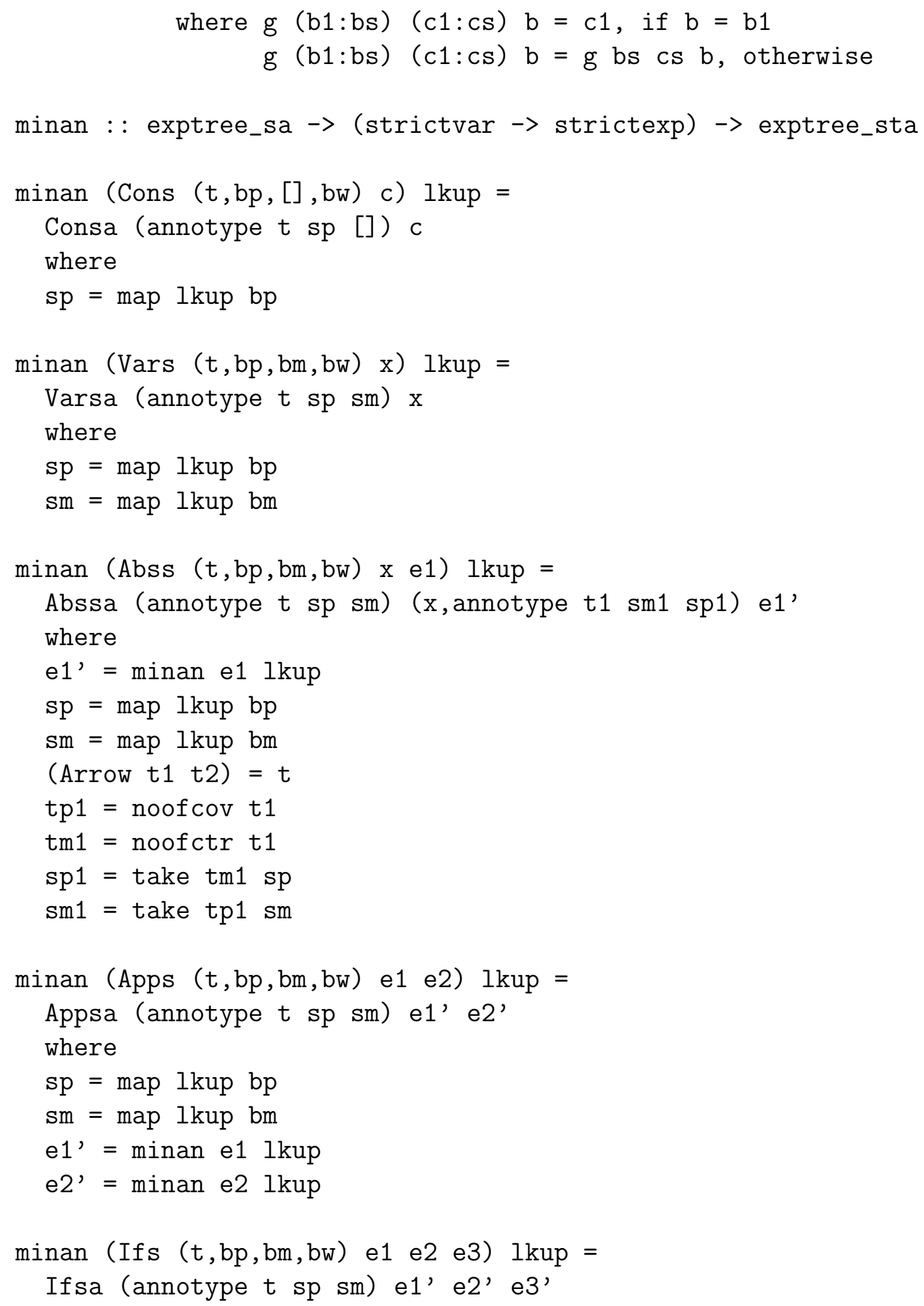




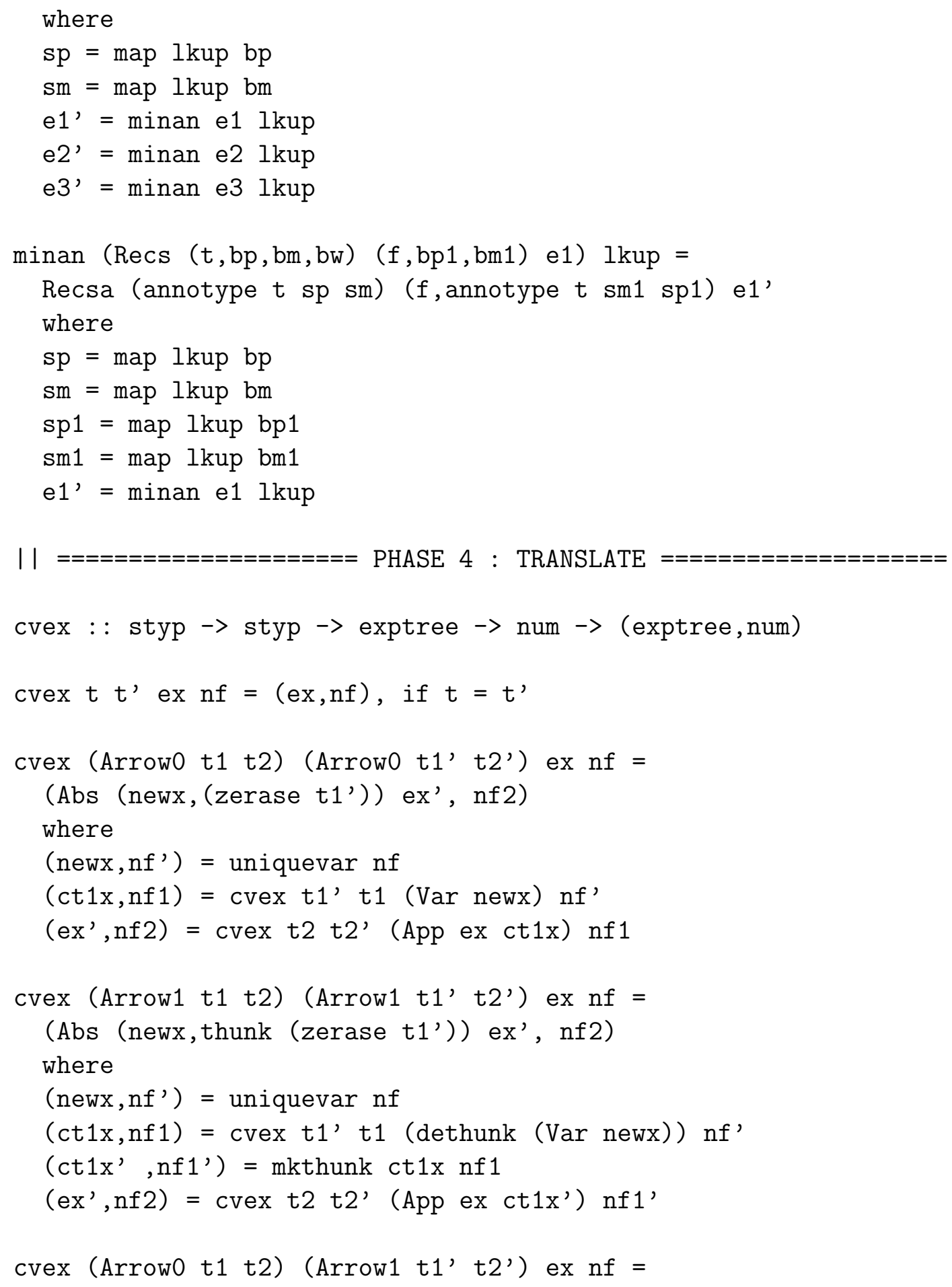




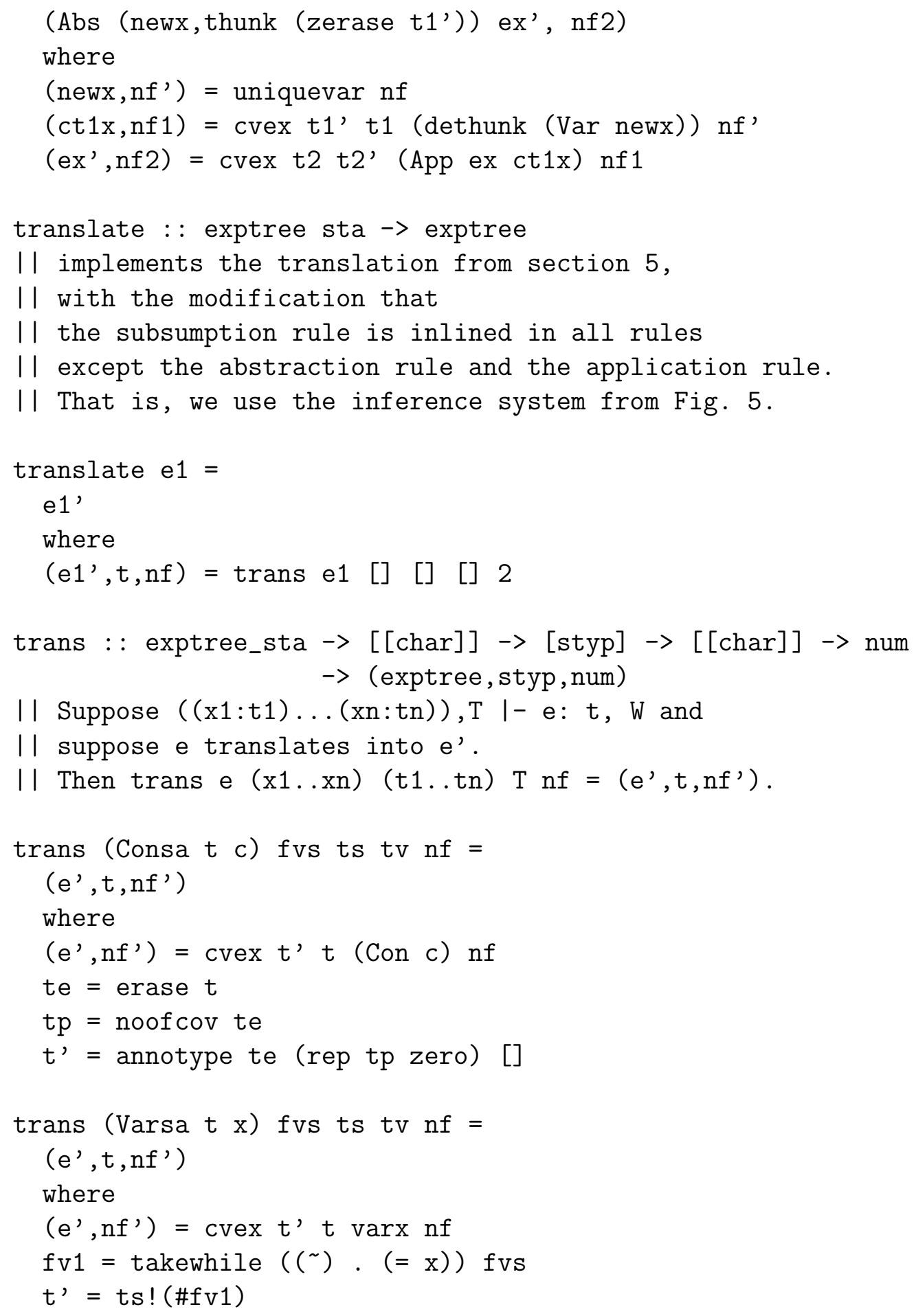




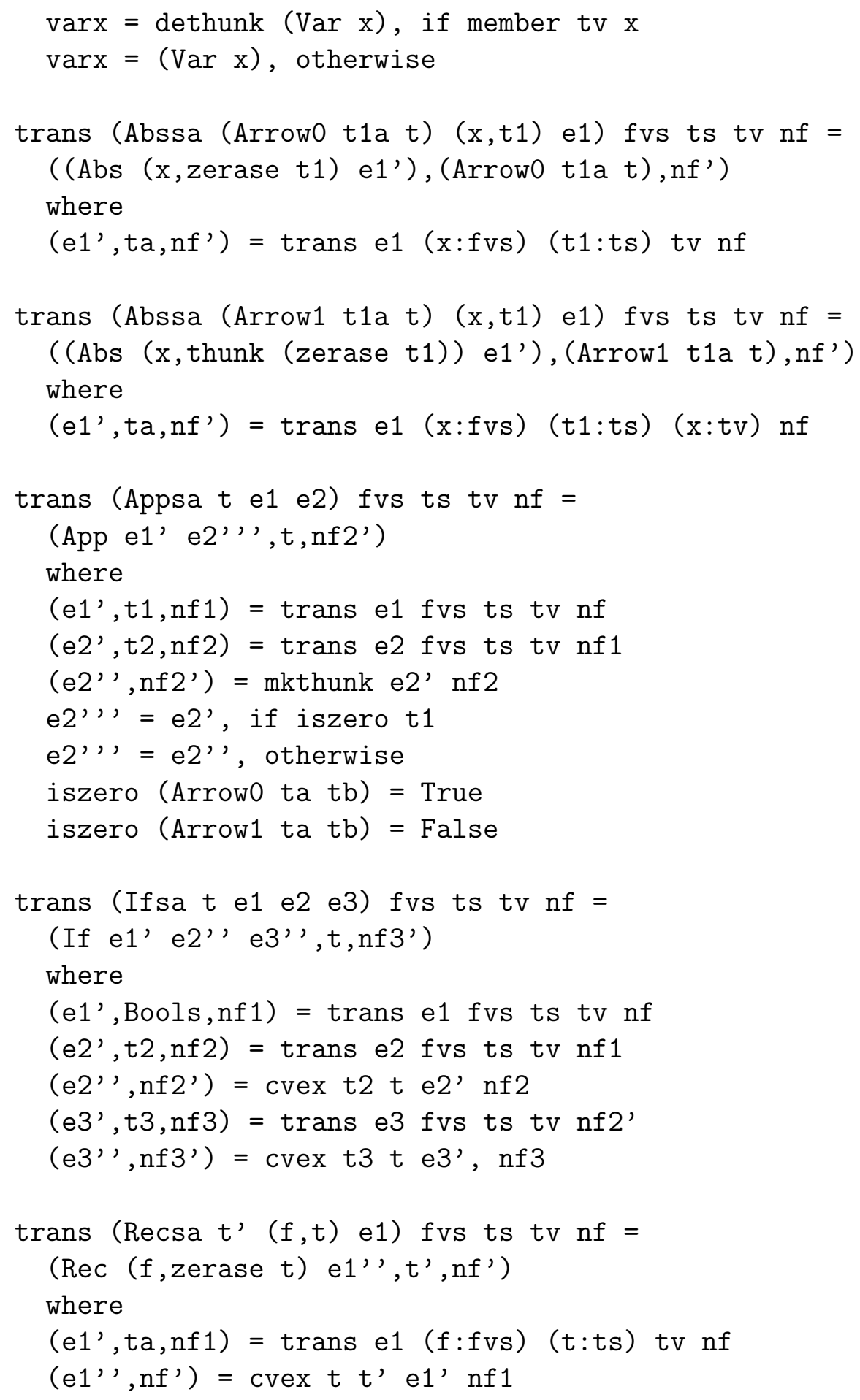




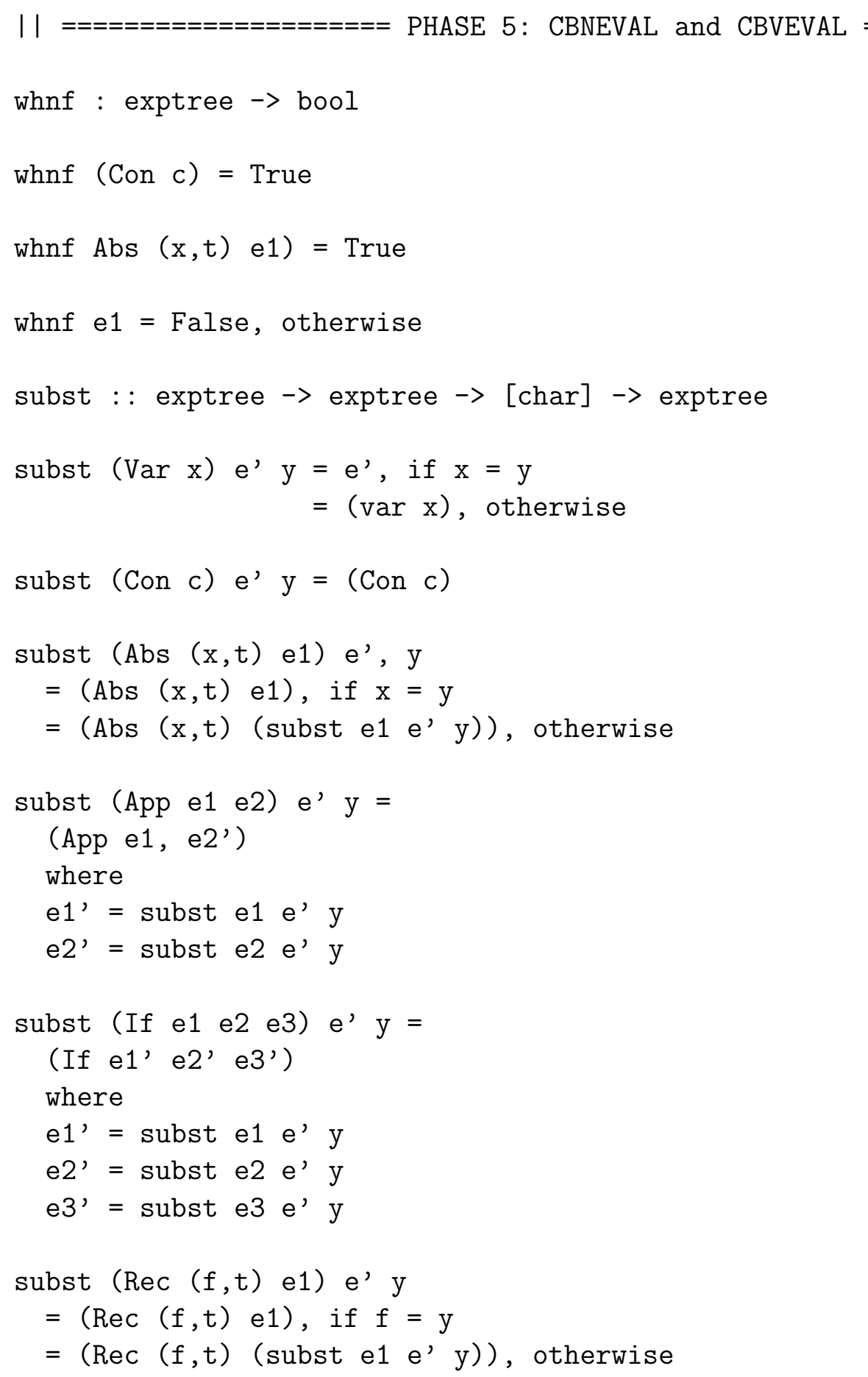




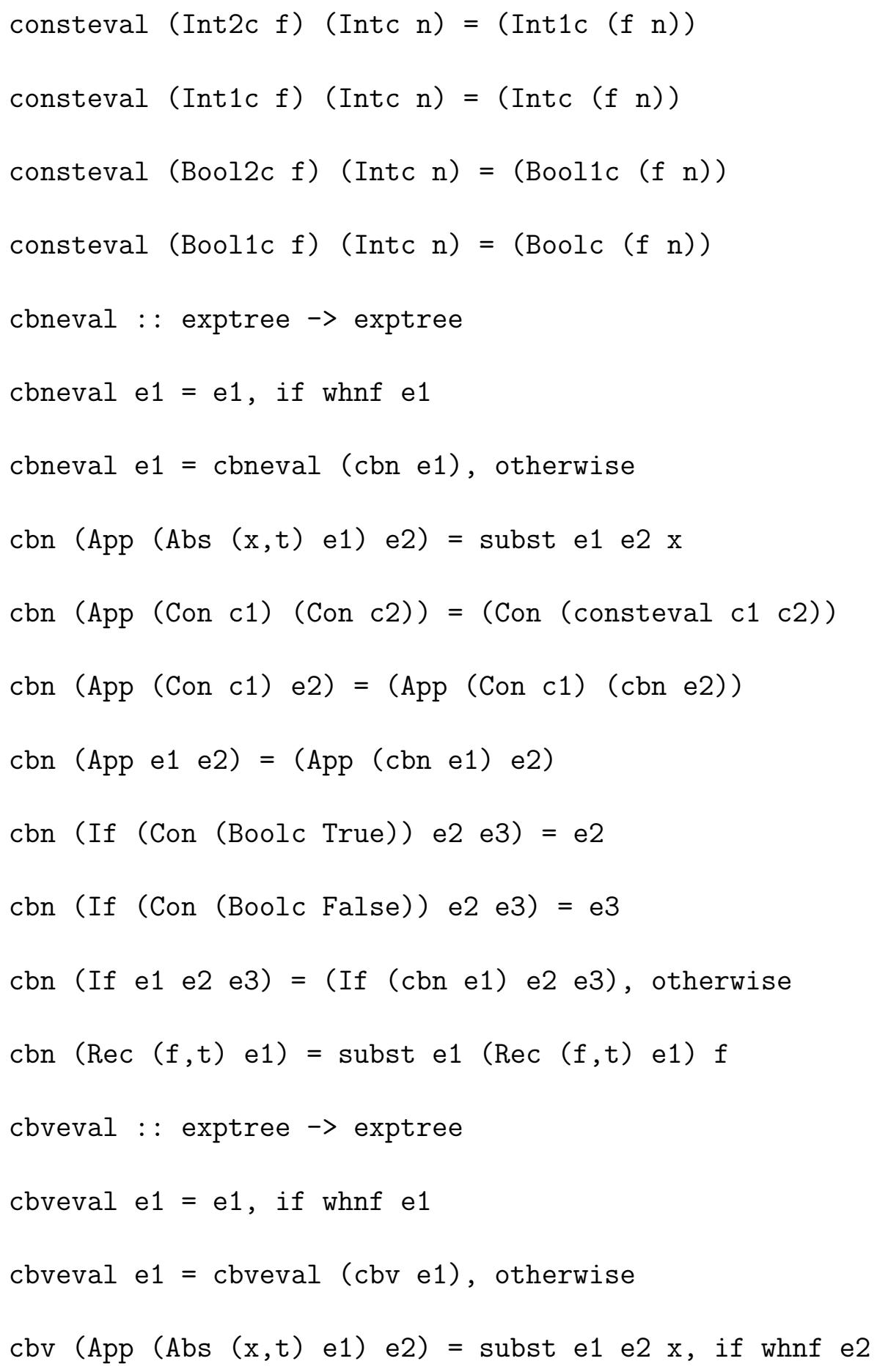




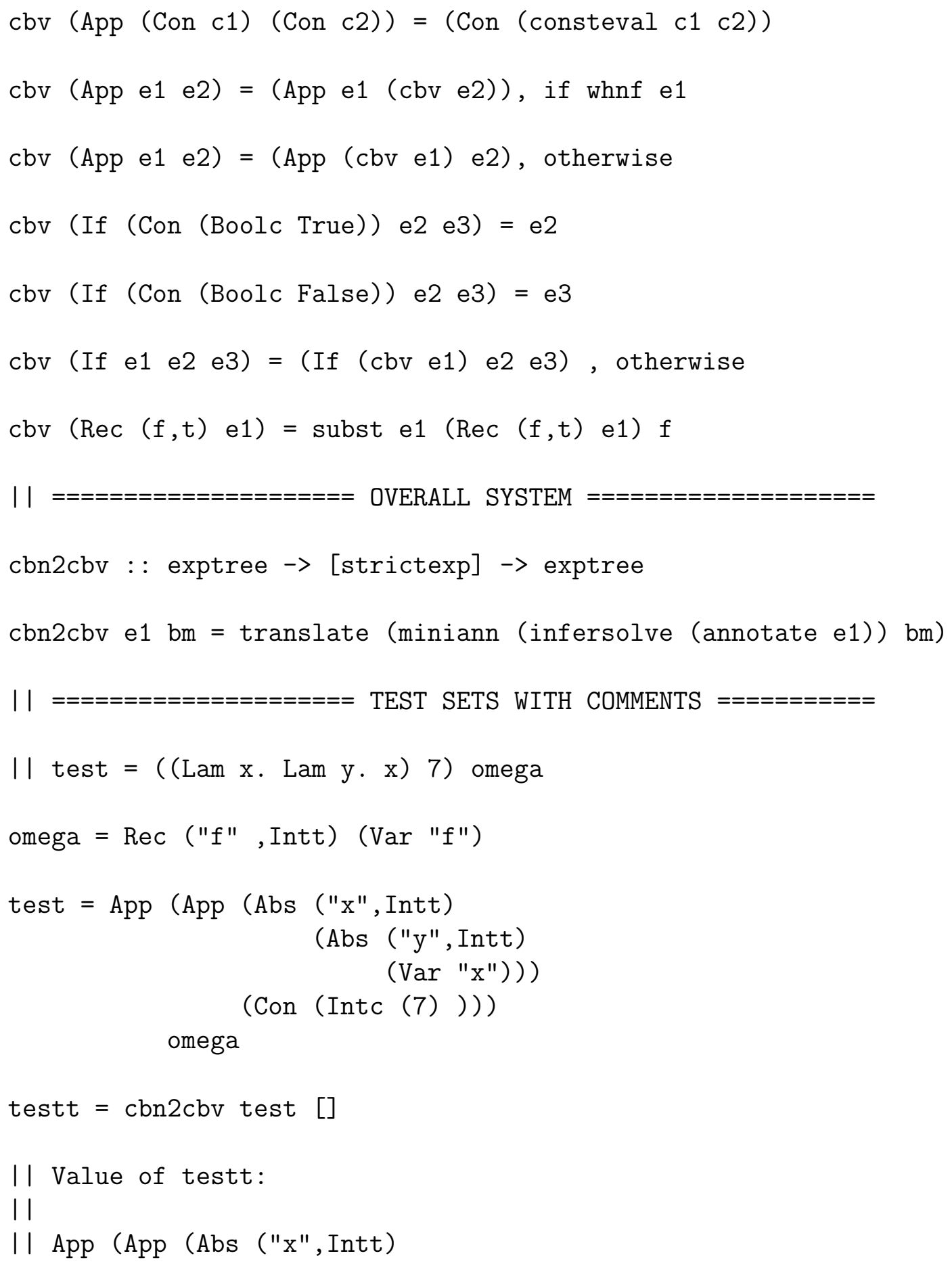




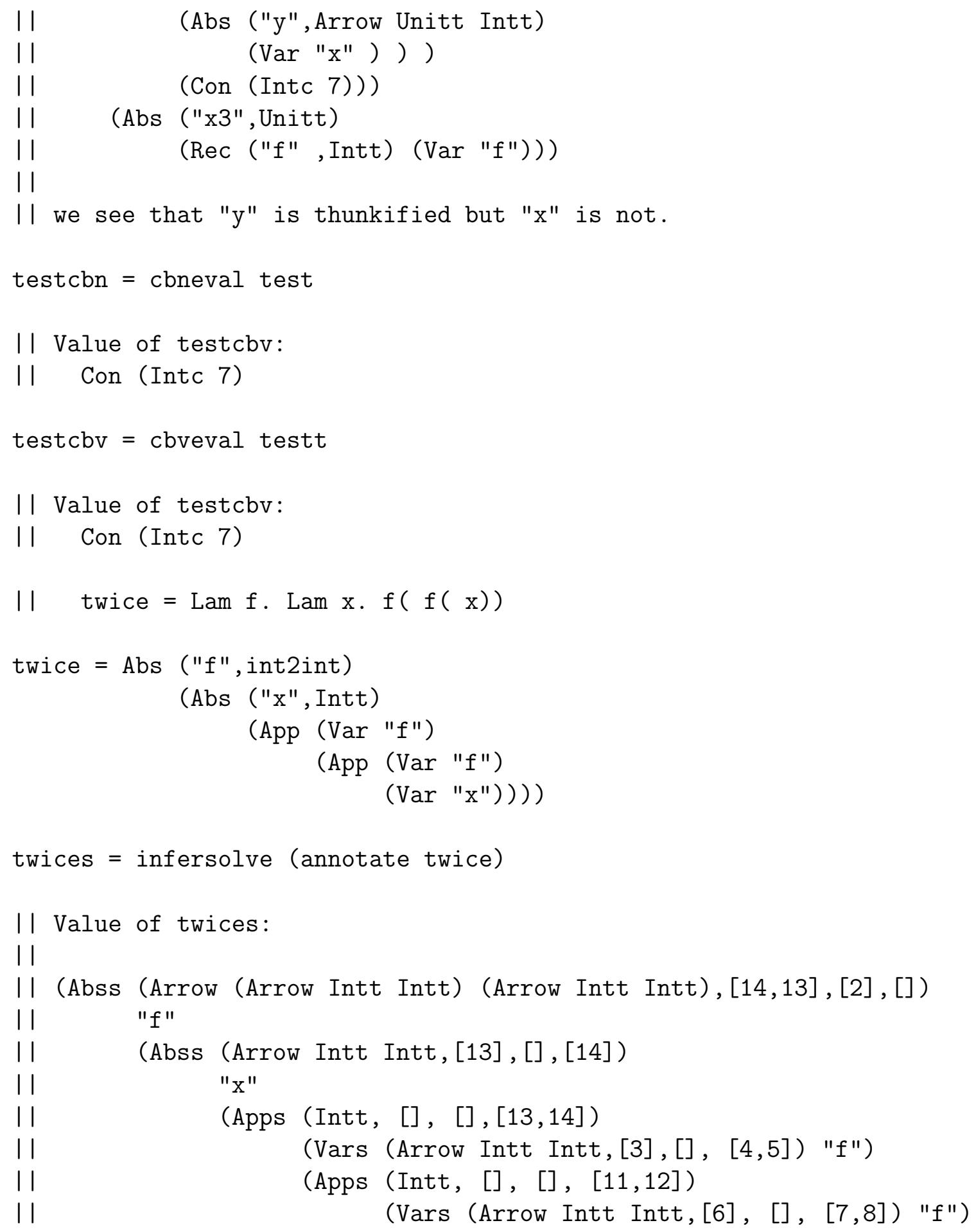




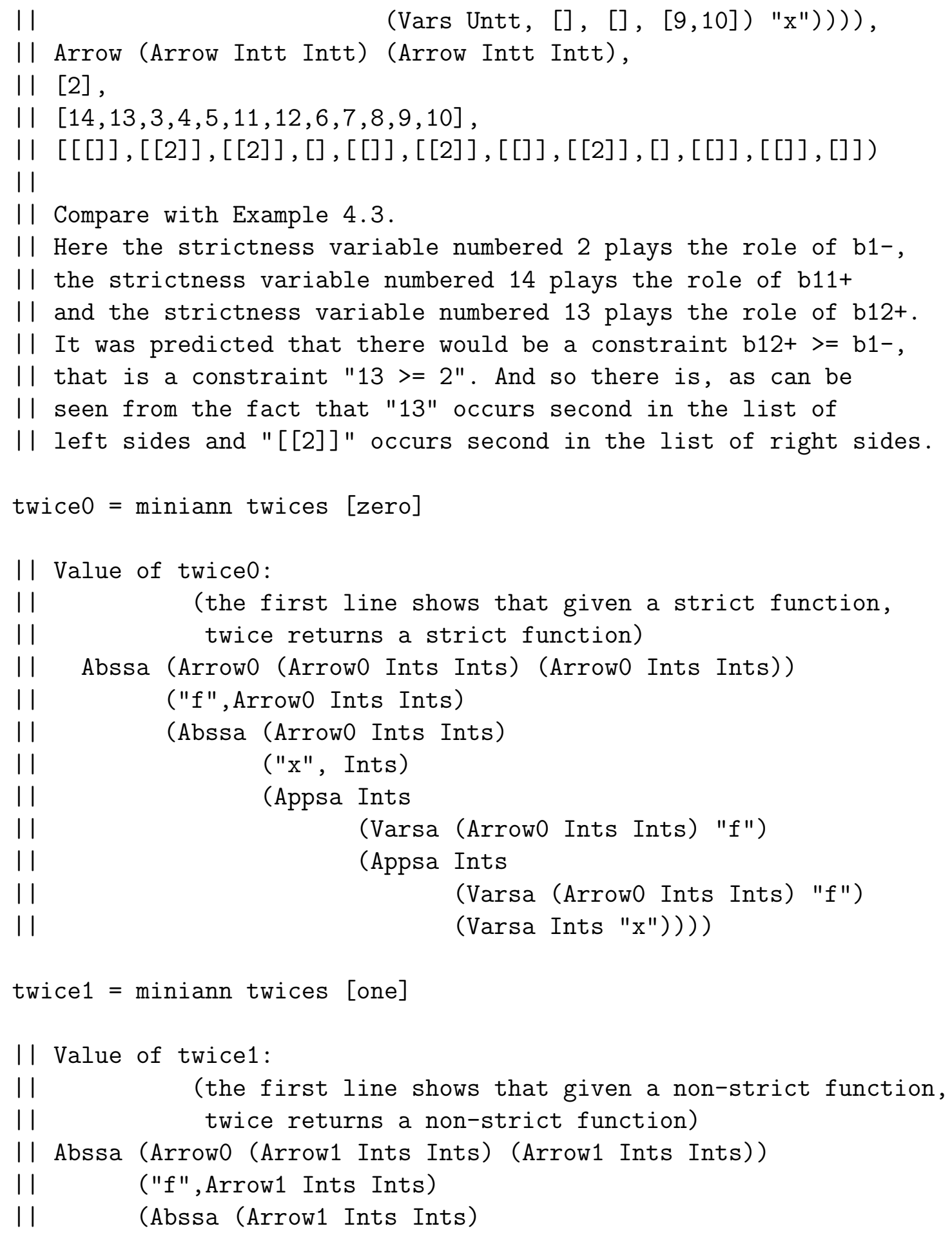




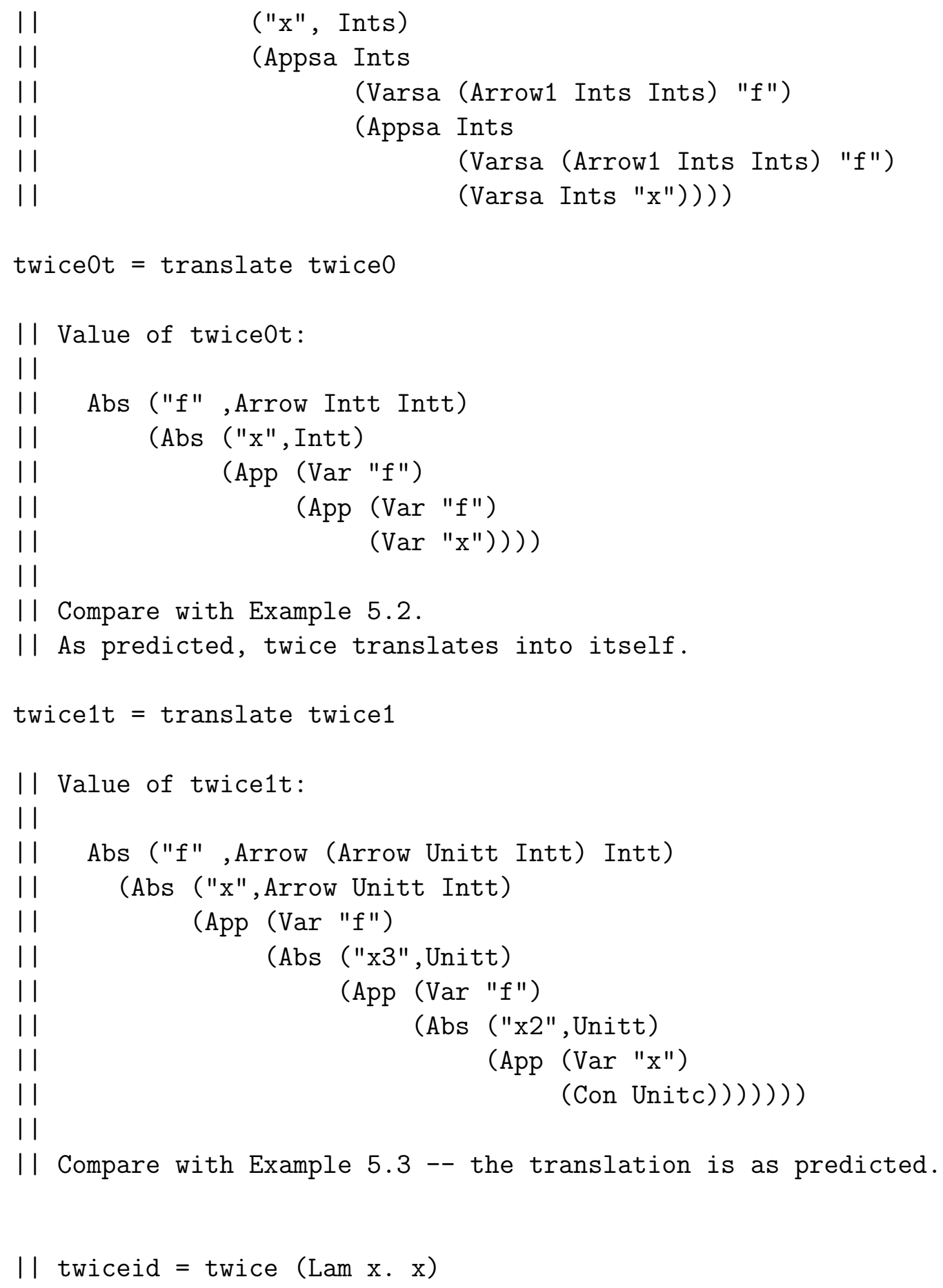




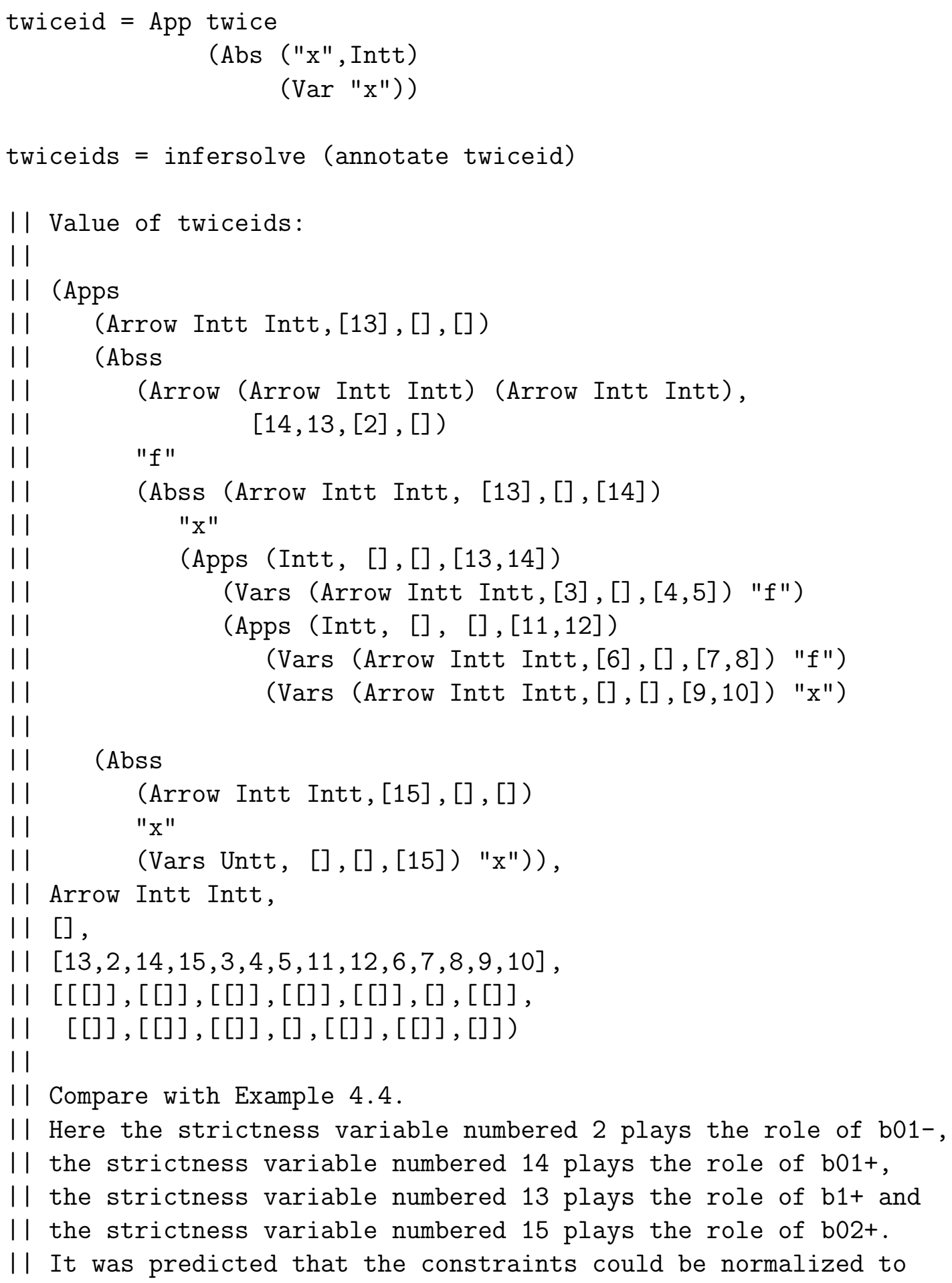




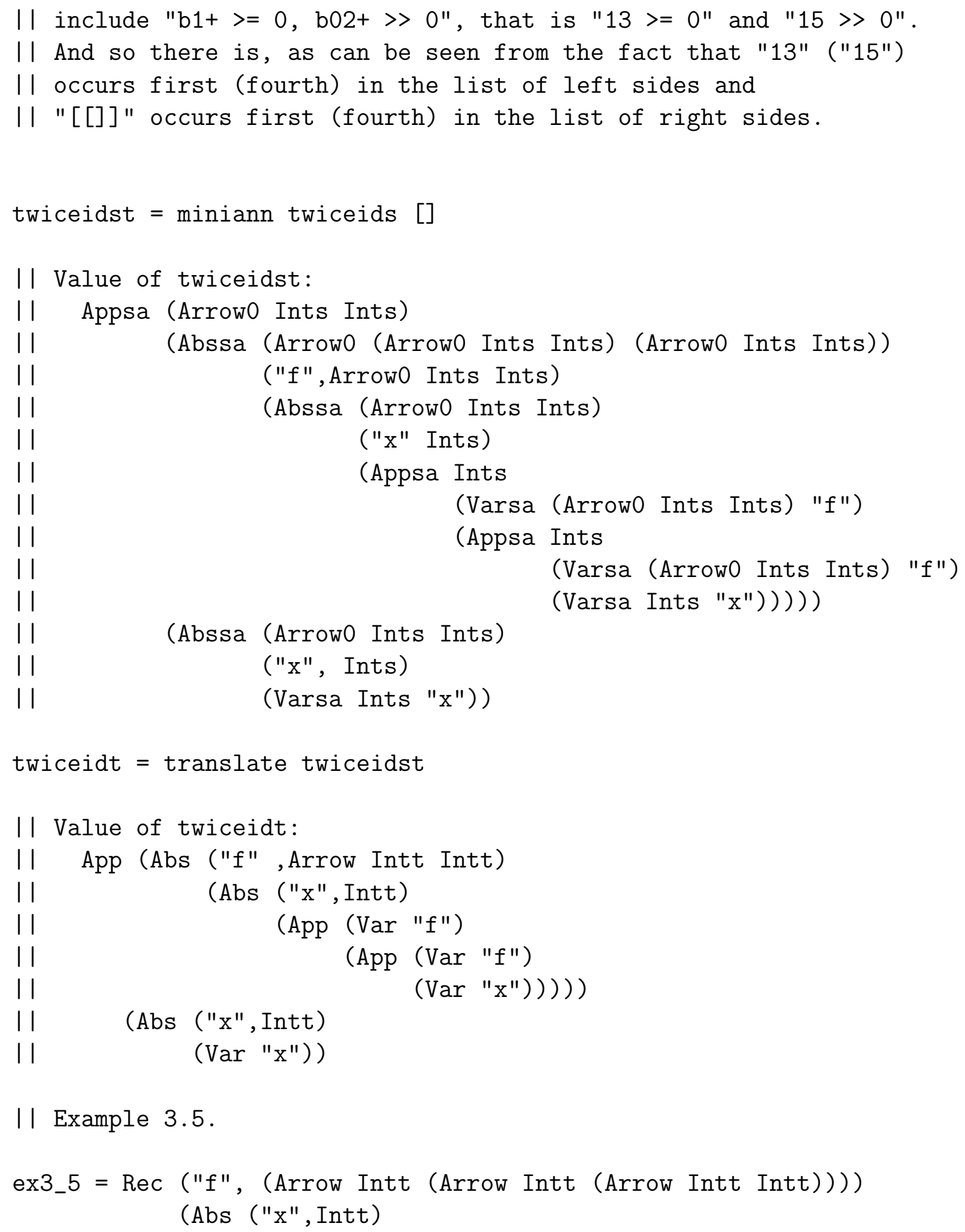




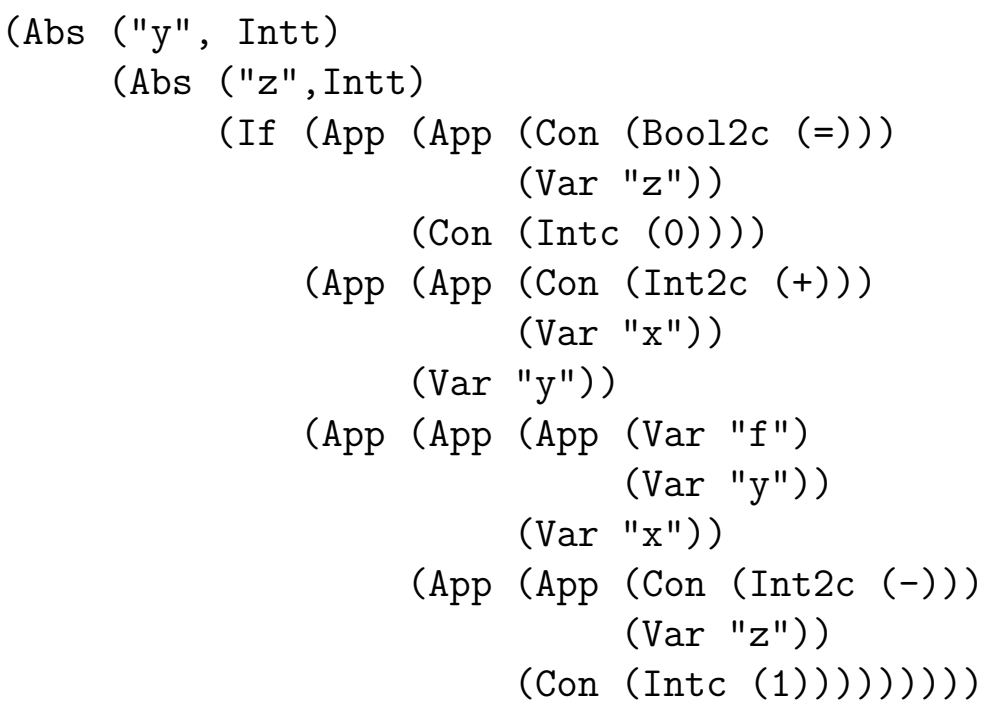


II Example 3.6.

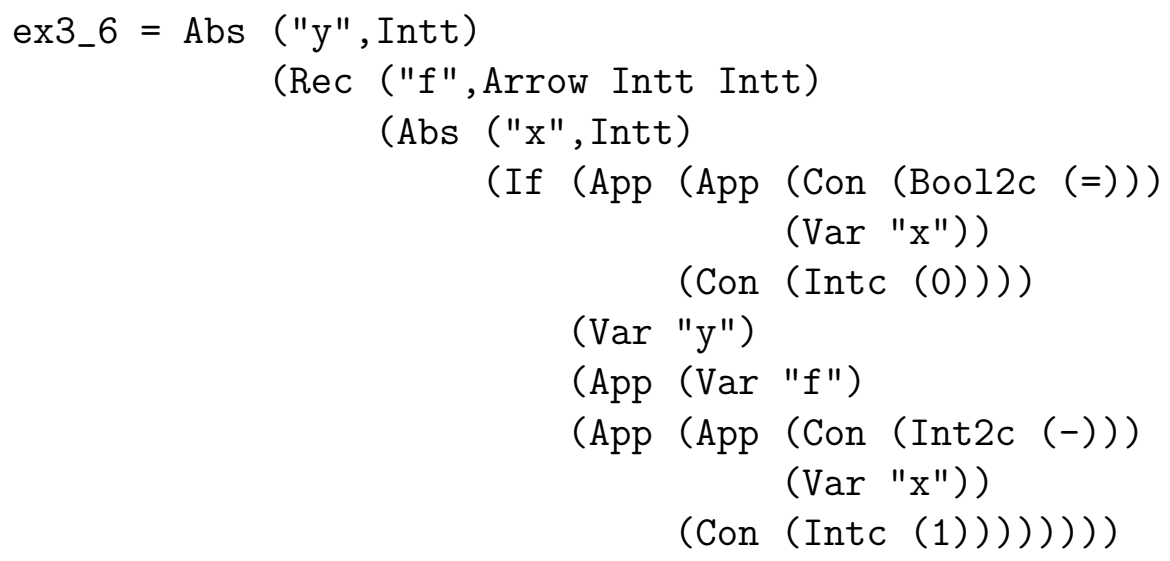




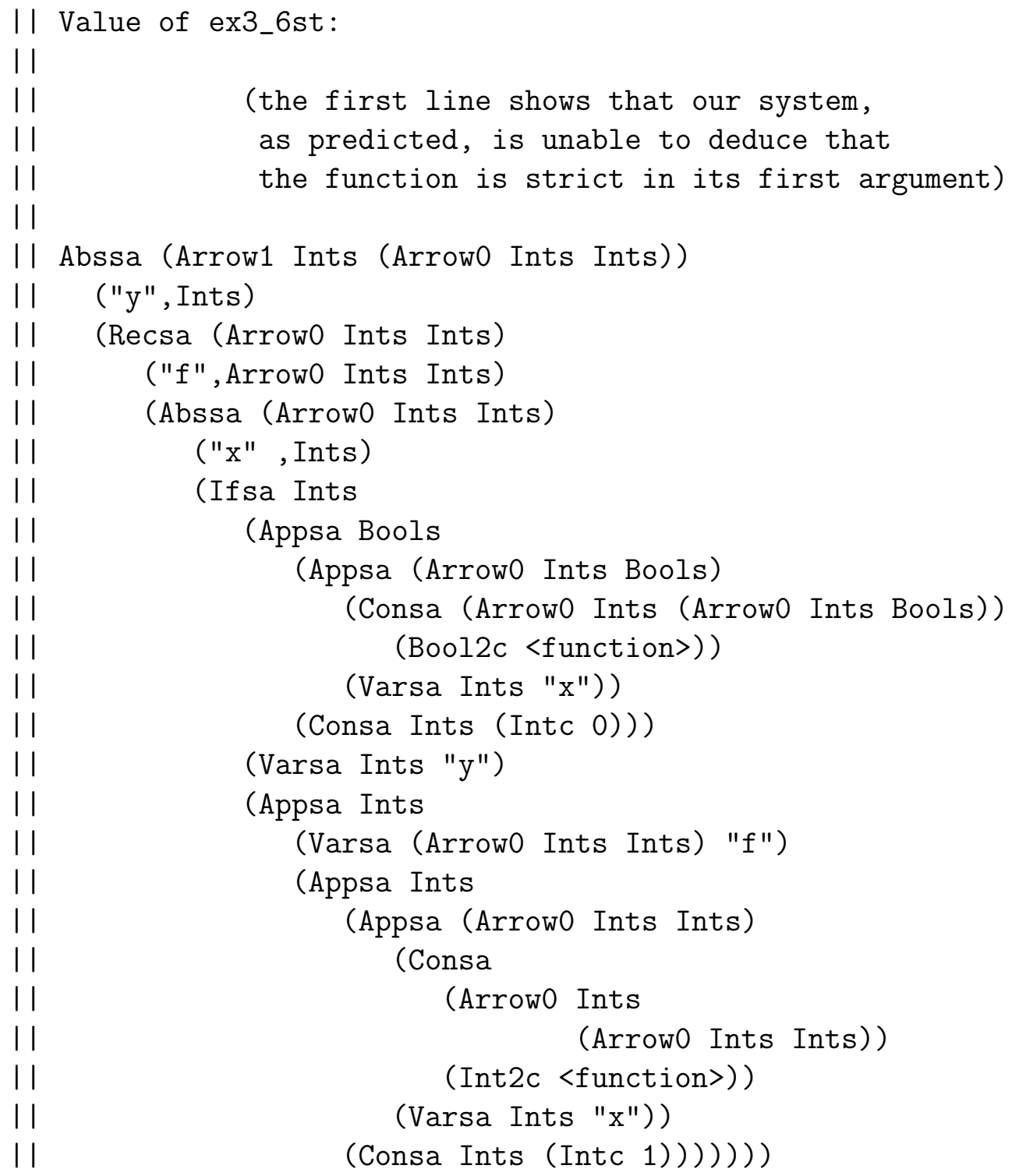

